\title{
DOUBLE VARIATIONAL PRINCIPLE FOR MEAN DIMENSION
}

\author{
ELON LINDENSTRAUSS, MASAKI TSUKAMOTO
}

\begin{abstract}
We develop a variational principle between mean dimension theory and rate distortion theory. We consider a minimax problem about the rate distortion dimension with respect to two variables (metrics and measures). We prove that the minimax value is equal to the mean dimension for a dynamical system with the marker property. The proof exhibits a new combination of ergodic theory, rate distortion theory and geometric measure theory. Along the way of the proof, we also show that if a dynamical system has the marker property then it has a metric for which the upper metric mean dimension is equal to the mean dimension.
\end{abstract}

\section{INTRODUCTION}

1.1. Statement of the main result. The purpose of this paper is to develop a new variational principle in dynamical systems theory. We first quickly prepare the terminologies and state the main result. Backgrounds will be explained in $\$ 1.2$.

A pair $(\mathcal{X}, T)$ is called a dynamical system if $\mathcal{X}$ is a compact metrizable space and $T: \mathcal{X} \rightarrow \mathcal{X}$ is a homeomorphism. We denote by $\mathscr{M}^{T}(\mathcal{X})$ the set of $T$-invariant Borel probability measures on $\mathcal{X}$. The standard variational principle ([Goodw69, D70, Goodm71]) states that the topological entropy $h_{\text {top }}(T)$ is equal to the supremum of the ergodic-theoretic entropy $h_{\mu}(T)$ over $\mu \in \mathscr{M}^{T}(\mathcal{X})$ :

$$
h_{\text {top }}(T)=\sup _{\mu \in \mathscr{M}^{T}(\mathcal{X})} h_{\mu}(T) .
$$

Our main result below is an analogous formula in mean dimension theory.

Date: January 18, 2019.

2010 Mathematics Subject Classification. 37A05, 37B99, 94A34.

Key words and phrases. dynamical system, mean dimension, rate distortion dimension, variational principle, invariant measure, geometric measure theory.

E.L was partially supported by ISF grant $891 / 15$. M.T. was partially supported by JSPS KAKENHI 18K03275. 
Mean dimension (denoted by $\operatorname{mdim}(\mathcal{X}, T)$ ) is a topological invariant of dynamical systems introduced by Gromov [Gro99]. It counts how many parameters per iterate we need to describe an orbit in $(\mathcal{X}, T)$. We review its definition in $\$ 2.1$. We would like to connect mean dimension to some information-theoretic quantity as in (1.1). An appropriate notion turns out to be rate distortion dimension, which was first introduced by Kawabata-Dembo KD94.

Let $\mathscr{D}(\mathcal{X})$ be the set of metrics (i.e. distance functions) on $\mathcal{X}$ compatible with the topology. Take $d \in \mathscr{D}(\mathcal{X})$ and $\mu \in \mathscr{M}^{T}(\mathcal{X})$. Consider a stochastic process $\left\{T^{n} x\right\}_{n \in \mathbb{Z}}$ where $x \in \mathcal{X}$ is chosen randomly according to $\mu$. We denote by $R(d, \mu, \varepsilon), \varepsilon>0$, the rate distortion function of this process with respect to the distortion measure $d$. This evaluates how many bits per iterate we need to describe the process within the distortion (w.r.t. $d$ ) bound by $\varepsilon$. We review its definition in $\$ 2.3$. We define the upper/lower rate distortion dimensions by 1

$$
\begin{aligned}
& \overline{\operatorname{rdim}}(\mathcal{X}, T, d, \mu)=\limsup _{\varepsilon \rightarrow 0} \frac{R(d, \mu, \varepsilon)}{\log (1 / \varepsilon)}, \\
& \underline{\operatorname{rdim}}(\mathcal{X}, T, d, \mu)=\liminf _{\varepsilon \rightarrow 0} \frac{R(d, \mu, \varepsilon)}{\log (1 / \varepsilon)} .
\end{aligned}
$$

When the upper and lower limits coincide, we denote their common value by $\operatorname{rdim}(\mathcal{X}, T, d, \mu)$.

A dynamical system $(\mathcal{X}, T)$ is said to have the marker property if for any $N>0$ there exists an open set $U \subset \mathcal{X}$ satisfying

$$
\mathcal{X}=\bigcup_{n \in \mathbb{Z}} T^{-n} U, \quad U \cap T^{-n} U=\emptyset \quad(\forall 1 \leq n \leq N) .
$$

This property implies that $(\mathcal{X}, T)$ is free (i.e. it has no periodic points). Free minimal systems and their extensions have the marker property. The marker property has been intensively used in the context of the embedding problem (see \$1.2) and related issues [Lin99, GLT16, GT, GQT.

Now we can state our main result.

Theorem 1.1 (Double Variational Principle). If a dynamical system $(\mathcal{X}, T)$ has the marker property, then

$$
\begin{aligned}
\operatorname{mdim}(\mathcal{X}, T) & =\min _{d \in \mathscr{D}(\mathcal{X})} \sup _{\mu \in \mathscr{M}^{T}(\mathcal{X})} \overline{\operatorname{rdim}}(\mathcal{X}, T, d, \mu) \\
& =\min _{d \in \mathscr{D}(\mathcal{X})} \sup _{\mu \in \mathscr{M}^{T}(\mathcal{X})} \underline{\operatorname{rdim}}(\mathcal{X}, T, d, \mu) .
\end{aligned}
$$

\footnotetext{
${ }^{1}$ Throughout the paper we assume that the base of the logarithm is two. The natural logarithm (i.e. the logarithm of base $e$ ) is written as $\ln (\cdot)$.
} 
Here "min" indicates that the minimum is attained by somed.

A fundamental difference between the standard variational principle (1.1) and our new one (1.3) is that (1.1) is a maximazation problem with respect to the single variable $\mu$ wheres (1.3) is a minimax problem with respect to the two variables $d$ and $\mu$. We have used the word "double" in order to emphasize that there exist two variables playing different roles.

Remark 1.2. (1) We have adopted the minimax approach in (1.3). It might also look interesting to consider a maximin approach:

$$
\begin{aligned}
& \sup _{\mu \in \mathscr{M}^{T}(\mathcal{X})} \inf _{d \in \mathscr{D}(\mathcal{X})} \overline{\operatorname{rdim}}(\mathcal{X}, T, d, \mu), \\
& \sup _{\mu \in \mathscr{M}^{T}(\mathcal{X})} \inf _{d \in \mathscr{D}(\mathcal{X})} \underline{\operatorname{rdim}}(\mathcal{X}, T, d, \mu) .
\end{aligned}
$$

However this turns out to be fruitless. Indeed both the quantities in (1.4) are always zero. More strongly, we can prove that for any dynamical system $(\mathcal{X}, T)$ and $\mu \in \mathscr{M}^{T}(\mathcal{X})$ there exists $d \in \mathscr{D}(\mathcal{X})$ satisfying $\overline{\operatorname{rdim}}(\mathcal{X}, T, d, \mu)=0$.

(2) The rate distortion dimension depends on both metrics $d$ and measures $\mu$. It might look more satisfactory to define a certain "ergodic-theoretic mean dimension" in a purely measuretheoretic way and prove a corresponding "variational principle" for mean dimension. But this naive approach is impossible: Let us consider an arbitrary ergodic measure-preserving system. By the Jewett-Krieger theorem [J70, Kri70] we can find a dynamical system $(\mathcal{X}, T)$ which has only one invariant probability measure (say, $\mu$ ) and that $(\mathcal{X}, \mu, T)$ is measure-theoretically isomorphic to the given system. It is known that uniquely ergodic systems have zero mean dimension [LW00, Theorem 5.4]. So, if we have a "variational principle", the given measure-preserving system must have zero "ergodic-theoretic mean dimension".

(3) We conjecture that the marker property assumption in Theorem 1.1 is, in fact, unnecessary. The proof of Theorem 1.1 shows that the inequality

$$
\operatorname{mdim}(\mathcal{X}, T) \leq \inf _{d \in \mathscr{D}(\mathcal{X})} \sup _{\mu \in \mathscr{M}^{T}(\mathcal{X})} \underline{\operatorname{rdim}}(\mathcal{X}, T, d, \mu)
$$

holds true for any dynamical system $(\mathcal{X}, T)$. So the remaining problem is how to prove the reverse inequality. See 1.4 for further discussions. 
1.2. Backgrounds. Mean dimension provides a nontrivial information for infinite dimensional dynamical systems of infinite topological entropy. It has several applications which cannot be touched within the framework of topological entropy [LW00, Lin99, GLT16, GT, GQT, MT. As an illustration, we explain an application to the problem of embedding dynamical systems into shift actions.

Consider the $N$-dimensional cube $C_{N}:=[0,1]^{N}$ and let $\sigma:\left(C_{N}\right)^{\mathbb{Z}} \rightarrow$ $\left(C_{N}\right)^{\mathbb{Z}}$ be the shift on the alphabet $C_{N}$ where $\left(C_{N}\right)^{\mathbb{Z}}$ is endowed with the standard product topology. The mean dimension of $\left(\left(C_{N}\right)^{\mathbb{Z}}, \sigma\right)$ is $N$. Given a dynamical system $(\mathcal{X}, T)$, we are interested in whether we can embed 2 it into $\left(\left(C_{N}\right)^{\mathbb{Z}}, \sigma\right)$ or not.

Periodic points are an obvious obstruction: If $(\mathcal{X}, T)$ has too many periodic points (e.g. if the set of fixed points has dimension greater than $N$ ) then it cannot be embedded into $\left(C_{N}\right)^{\mathbb{Z}}$. Mean dimension provides another obstruction: If we can embed $(\mathcal{X}, T)$ into $\left(C_{N}\right)^{\mathbb{Z}}$ then $\operatorname{mdim}(\mathcal{X}, T) \leq \operatorname{mdim}\left(\left(C_{N}\right)^{\mathbb{Z}}, \sigma\right)=N$. We can construct free (and, moreover, minimal) dynamical systems of arbitrary mean dimension [LW00, Proposition 3.5]. So there exist plenty of examples which are free but cannot be embedded into $\left(C_{N}\right)^{\mathbb{Z}}$. (This observation by [LW00] solved a question posed by Auslander in 1970s.)

Somehow surprisingly, a partial converse also holds. Based on the work [Lin99, the papers [GT, GQT 3 proved that if $(\mathcal{X}, T)$ has the marker property and satisfies $\operatorname{mdim}(\mathcal{X}, T)<N / 2$ then we can embed it into $\left(C_{N}\right)^{\mathbb{Z}}$. The example in [LT14 shows that the condition $\operatorname{mim}(\mathcal{X}, T)<N / 2$ is optimal. These results demonstrate that mean dimension is certainly a reasonable measure of the "size" of dynamical systems.

It is classically known that the concepts of entropy and dimension are closely connected (Rényi Rén59], Kolmogorov-Tihomirov [KT63] and Kawabata-Dembo KD94] ${ }^{4}$. So it is natural to expect that we can approach to mean dimension from the entropy theory viewpoint. The first attempt of such an approach was made by Weiss and the first named author [LW00] by introducing the notion of metric mean dimension. This is a dynamical analogue of Minkowski dimension defined as follows. Let $(\mathcal{X}, T)$ be a dynamical system with a metric $d$.

\footnotetext{
${ }^{2} f: \mathcal{X} \rightarrow\left(C_{N}\right)^{\mathbb{Z}}$ is called an embedding of a dynamical system if it is a topological embedding and satisfies $f \circ T=\sigma \circ f$.

${ }^{3}$ The papers [GT, GQT used the ideas of communication theory and signal processing. This is another manifestation of the intimate connections between mean dimension and information theory.

${ }^{4}$ It seems that these attract new interests of information theory researchers in the context of compressed sensing; see, e.g. WV10 and RJEP.
} 
Let $S(\mathcal{X}, T, d, \varepsilon)$ be its entropy detected at the resolution $\varepsilon>0$. (See $\$ 2.1$ for the precise definition.) The topological entropy is given by $h_{\text {top }}(T)=\lim _{\varepsilon \rightarrow 0} S(\mathcal{X}, T, d, \varepsilon)$. We define the upper/lower metric mean dimensions by

$$
\begin{aligned}
& \overline{\operatorname{mdim}}_{\mathrm{M}}(\mathcal{X}, T, d)=\limsup _{\varepsilon \rightarrow 0} \frac{S(\mathcal{X}, T, d, \varepsilon)}{\log (1 / \varepsilon)}, \\
& \underline{\operatorname{mdim}}_{\mathrm{M}}(\mathcal{X}, T, d)=\liminf _{\varepsilon \rightarrow 0} \frac{S(\mathcal{X}, T, d, \varepsilon)}{\log (1 / \varepsilon)} .
\end{aligned}
$$

When the upper and lower limits coincide, we denote their common value by $\operatorname{mdim}_{\mathrm{M}}(\mathcal{X}, T, d)$. In analogy with the well-known fact that Minkowski dimension bounds topological dimension, we have [LW00, Theorem 4.2]

$$
\operatorname{mdim}(\mathcal{X}, T) \leq \underline{\operatorname{mdim}}_{\mathrm{M}}(\mathcal{X}, T, d) \leq \overline{\operatorname{mdim}}_{\mathrm{M}}(\mathcal{X}, T, d) .
$$

It was also proved in [Lin99, Theorem 4.3] that if $(\mathcal{X}, T)$ has the marker property then there exists a metric $d$ on $\mathcal{X}$ satisfying $\operatorname{mdim}(\mathcal{X}, T)=$ $\underline{\operatorname{mdim}}_{M}(\mathcal{X}, T, d)$. The same statement for upper metric mean dimension remained open since [Lin99]. We will establish it as a part of the proof of Theorem 1.1, See Theorem 3.12 below.

Metric mean dimension seems to be quite useful. In particular, it provides a powerful method to obtain upper bounds on mean dimension via (1.6). This was used for example in [T18a] for solving a problem of Gromov Gro99] to estimate the mean dimension of a dynamical system in holomorphic curve theory. It also has an application to the study of expansive group actions $\mathrm{MT}$.

It seems desirable to inject ergodic theory and in particular invariant measures into mean dimension theory in order to broaden the scope of applications. This motivated the authors to begin the study of our previous paper [LT18]. In [LT18] we proved the following variational principle between metric mean dimension and rate distortion function under a mild condition on $d$ (called tame growth of covering numbers; see Definition 3.8):

$$
\begin{aligned}
& \overline{\operatorname{mdim}}_{\mathrm{M}}(\mathcal{X}, T, d)=\limsup _{\varepsilon \rightarrow 0} \frac{\sup _{\mu \in \mathscr{M}^{T}(\mathcal{X})} R(d, \mu, \varepsilon)}{\log (1 / \varepsilon)}, \\
& \underline{\operatorname{mim}}_{\mathrm{M}}(\mathcal{X}, T, d)=\liminf _{\varepsilon \rightarrow 0} \frac{\sup _{\mu \in \mathscr{M}^{T}(\mathcal{X})} R(d, \mu, \varepsilon)}{\log (1 / \varepsilon)} .
\end{aligned}
$$

We proved this by developing a rate distortion theory version of Misiurewicz's proof [Mis76] of the standard variational principle (1.1). This is an initial step of our program to inject measure into mean dimension theory. However it is still not completely satisfactory. The equation 
(1.7) implies that we can construct $\mu \in \mathcal{M}^{T}(\mathcal{X})$ capturing (most of) dynamical complexity of $(\mathcal{X}, T)$ at each fixed resolution $\varepsilon>0$. It would be nicer if we could find $\mu$ capturing the dynamical complexity over all resolutions. In other words, we would like to exchange the order of the limit and supremum in (1.7). This naturally leads us to the following question (this was also posed by Velozo-Velozo [VV], Section 6]):

Problem 1.3. When do the following equalities hold?

$$
\begin{aligned}
& \overline{\operatorname{mdim}}_{\mathrm{M}}(\mathcal{X}, T, d)=\sup _{\mu \in \mathscr{M}^{T}(\mathcal{X})} \overline{\operatorname{rdim}}(\mathcal{X}, T, d, \mu), \\
& \underline{\operatorname{mim}}_{\mathrm{M}}(\mathcal{X}, T, d)=\sup _{\mu \in \mathscr{M}^{T}(\mathcal{X})} \underline{\operatorname{rdim}}(\mathcal{X}, T, d, \mu) .
\end{aligned}
$$

Of course, (1.8) does not hold in general. The following example clarifies the situation:

Example 1.4. Let $A=\{1,1 / 2,1 / 3, \ldots\} \cup\{0\} \subset[0,1]$ and $\mathcal{X}=A^{\mathbb{Z}}$ with the shift $\sigma$. Define a metric $d$ on $\mathcal{X}$ by $d(x, y)=\sum_{n \in \mathbb{Z}} 2^{-|n|} \mid x_{n}-$ $y_{n} \mid$. Then it is straightforward to check that $\operatorname{mdim}_{\mathrm{M}}(\mathcal{X}, \sigma, d)=1 / 2$ and that $\operatorname{rdim}(\mathcal{X}, \sigma, d, \mu)=0$ for any $\sigma$-invariant probability measure $\mu$ (cf. [KD94, Lemma 3.1]). So (1.8) does not hold even for this simple example. However we can push our consideration further. Let $B=$ $\left\{1,2^{-1}, 2^{-2}, \ldots\right\} \cup\{0\}$ and consider a homeomorphism $f: A \rightarrow B$ defined by $f(1 / n)=2^{-n}$ and $f(0)=0$. Define a new metric $d^{\prime}$ on $\mathcal{X}=A^{\mathbb{Z}}$ by $d^{\prime}(x, y)=\sum_{n \in \mathbb{Z}} 2^{-n}\left|f\left(x_{n}\right)-f\left(y_{n}\right)\right|$. Then we can check that

$$
\operatorname{mdim}_{\mathrm{M}}\left(\mathcal{X}, \sigma, d^{\prime}\right)=\sup _{\mu \in \mathscr{M}^{T}(\mathcal{X})} \operatorname{rdim}\left(\mathcal{X}, \sigma, d^{\prime}, \mu\right)=0 .
$$

In particular (1.8) holds true for $d^{\prime}$.

The above example shows that we can expect the equality (1.8) only for well-chosen metrics $d$. This suggests a new viewpoint: We cannot stick to a fixed metric d. We should regard $d$ as a variable and move both $d$ and $\mu$. The double variational principle (Theorem 1.1) is a crystallization of this idea.

The proof of Theorem 1.1 also provides a partial answer to Problem 1.3 (see Corollary 3.14): If $(\mathcal{X}, T)$ has the marker property, then there exists a metric $d$ on $\mathcal{X}$ such that all the following quantities are equal to each other:

$$
\begin{aligned}
& \operatorname{mdim}(\mathcal{X}, T), \quad \overline{\operatorname{mdim}}_{\mathrm{M}}(\mathcal{X}, T, d), \quad \underline{\operatorname{mdim}}_{\mathrm{M}}(\mathcal{X}, T, d), \\
& \sup _{\mu \in \mathscr{M}^{T}(\mathcal{X})} \overline{\operatorname{rdim}}(\mathcal{X}, T, d, \mu), \quad \sup _{\mu \in \mathscr{M}^{T}(\mathcal{X})} \underline{\operatorname{rdim}}(\mathcal{X}, T, d, \mu) .
\end{aligned}
$$


1.3. Outline of the proof of Theorem 1.1. Let $(\mathcal{X}, T)$ be a dynamical system. The proof of Theorem 1.1 consists of the following three steps:

Step 1 (Metric mean dimension bounds rate distortion dimension): For all $d \in \mathscr{D}(\mathcal{X})$ and $\mu \in \mathscr{M}^{T}(\mathcal{X})$

$$
\begin{aligned}
& \overline{\operatorname{rdim}}(\mathcal{X}, T, d, \mu) \leq \overline{\operatorname{mdim}}_{\mathrm{M}}(\mathcal{X}, T, d), \\
& \underline{\operatorname{rdim}}(\mathcal{X}, T, d, \mu) \leq \underline{\operatorname{mdim}}_{\mathrm{M}}(\mathcal{X}, T, d) .
\end{aligned}
$$

Step 2 (Constructing invariant measures encoding dynamical complexity): For all $d \in \mathscr{D}(\mathcal{X})$

$$
\operatorname{mdim}(\mathcal{X}, T) \leq \sup _{\mu \in \mathscr{M}^{T}(\mathcal{X})} \underline{\operatorname{rdim}}(\mathcal{X}, T, d, \mu) .
$$

Step 3 (Constructing nice metrics): Under the marker property assumption

$$
\exists d \in \mathscr{D}(\mathcal{X}): \quad \operatorname{mdim}(\mathcal{X}, T)=\overline{\operatorname{mdim}}_{\mathrm{M}}(\mathcal{X}, T, d) .
$$

We emphasize that the marker property is used only in this step.

Combining the above three steps, we get Theorem 1.1. Step 1 is easy to prove. (See \$3.2,) So the main issues are Steps 2 and 3.

About Step 2: Let $d$ be a metric on $\mathcal{X}$. In the proof of Step 2, we introduce a new notion called mean Hausdorff dimension (denoted by $\left.\operatorname{mdim}_{H}(\mathcal{X}, T, d)\right)$. This is a dynamical version of Hausdorff dimension. As is well known in geometric measure theory, Hausdorff dimension is more closely related to measure theory than Minkowski dimension. So it is natural to expect that its dynamical analogue is helpful to connect measure theory to mean dimensions. We decompose Step 2 into two smaller steps:

Step 2.1 (Mean Hausdorff dimension bounds mean dimension):

$$
\operatorname{mdim}(\mathcal{X}, T) \leq \operatorname{mdim}_{\mathrm{H}}(\mathcal{X}, T, d)
$$

Step 2.2 (Dynamical analogue of Frostman's lemma): Under a mild condition on d (the tame growth of covering numbers; see

\footnotetext{
${ }^{5}$ The idea of introducing mean Hausdorff dimension was partly motivated by the study of Kawabata-Dembo [KD94, Proposition 3.2]. Roughly speaking, their result [KD94, Proposition 3.2] corresponds to Step 2.2 for $(\mathcal{X}, T)=\left(A^{\mathbb{Z}}\right.$, shift $)$ with $A \subset \mathbb{R}^{n}$. In other words, Step 2.2 is a generalization of their result to arbitrary dynamical systems.

${ }^{6}$ There also exists a small issue about the tame growth of covering numbers condition. But we ignore it here
} 
Definition 3.8)

$$
\operatorname{mdim}_{\mathrm{H}}(\mathcal{X}, T, d) \leq \sup _{\mu \in \mathscr{M}^{T}(\mathcal{X})} \underline{\operatorname{rdim}}(\mathcal{X}, T, d, \mu) .
$$

Step 2.1 is a dynamical analogue of the fact that Hausdorff dimension bounds topological dimension. Its proof is given in $\$ 3.2$. Step 2.2 is the main part of Step 2. Frostman's lemma is a classical result in geometric measure theory. Roughly speaking, it claims that we can construct a probability measure which obeys the scaling law corresponding to the Hausdorff dimension. We establish Step 2.2 by combining Frostman's lemma with the techniques of our previous variational principle (1.7). It roughly goes as follows. For $n \geq 1$ we set $d_{n}(x, y)=\max _{0 \leq k<n} d\left(T^{k} x, T^{k} y\right)$. By using the geometric measure theory around Frostman's lemma, for each $n \geq 1$, we construct a (noninvariant) probability measure $\nu_{n}$ on $\mathcal{X}$ which captures the geometric complexity of $\left(\mathcal{X}, d_{n}\right)$ over all resolutions. Consider

$$
\mu_{n}=\frac{1}{n} \sum_{k=0}^{n-1} T_{*}^{k} \nu_{n} .
$$

From the compactness we can choose a subsequence $\mu_{n_{k}}$ which converges to some invariant probability measure (say, $\mu$ ). We apply to $\mu$ the rate distortion theory version of Misiurewicz's technique [Mis76] (developed in [LT18]) and prove that $\mu$ captures most of the dynamical complexity of $(\mathcal{X}, T)$ over all resolutions.

About Step 3: Step 3 is technically hard. As we briefly noted in $\$ 1.2$, it was already proved in [Lin99, Theorem 4.3] that if $(\mathcal{X}, T)$ has the marker property then

$$
\exists d \in \mathscr{D}(\mathcal{X}): \quad \operatorname{mdim}(\mathcal{X}, T)=\underline{\operatorname{mdim}}_{\mathrm{M}}(\mathcal{X}, T, d) .
$$

The claim of Step 3 looks very similar. But, in fact, it is much subtler and remained to be an open problem for about 20 years since [Lin99]. It is difficult to briefly explain the ideas of the proof. (See $\$ 5.1$ for more background.) Here we just remark that the above (1.9) (with Steps 1 and 2) are already enough for proving the equality for the lower rate distortion dimension:

$$
\operatorname{mdim}(\mathcal{X}, T)=\min _{d \in \mathscr{D}(\mathcal{X})} \sup _{\mu \in \mathscr{M}^{T}(\mathcal{X})} \underline{\operatorname{rdim}}(\mathcal{X}, T, d, \mu) .
$$

1.4. Open problems and future directions. The most important open problem is to remove the marker property assumption in Theorem 1.1 . 
Problem 1.5. Prove the double variational principle (1.3) for all dynamical systems.

As we explained in 91.3 , the marker property is used only in Step 3 of the proof of Theorem 1.1. So Problem 1.5 reduces to

Problem 1.6. Prove that for any dynamical system $(\mathcal{X}, T)$

$$
\exists d \in \mathscr{D}(\mathcal{X}): \quad \operatorname{mdim}(\mathcal{X}, T)=\overline{\operatorname{mdim}}_{\mathrm{M}}(\mathcal{X}, T, d) .
$$

We emphasize that the same problem for lower metric mean dimension is also open.

Problems 1.5 and 1.6 are certainly the central open problems. But there also exists a different interesting direction. Step 2 of the proof of Theorem 1.1 does not use the marker property assumption. So we always have the inequality

$$
\operatorname{mdim}(\mathcal{X}, T) \leq \inf _{d \in \mathscr{D}(\mathcal{X})} \sup _{\mu \in \mathscr{M}^{T}(\mathcal{X})} \underline{\operatorname{rdim}}(\mathcal{X}, T, d, \mu),
$$

although we don't know whether the equality holds or not. This implies that we can always find a "sufficiently rich" invariant measure $\mu$. Study of these measures for concrete examples seems very interesting. We begin such study in 8 . Although our investigation in this direction has just started, the result in $\$ 6$ seems to suggest a high potential of this research direction. It is desirable to study geometric examples in Gro99, T18a, T18b] from the viewpoint of the double variational principle.

1.5. Organization of the paper and how to read it. $\$ 2$ is a preparation of basics of mean dimension and rate distortion function. In $\$ 3$ we introduce mean Hausdorff dimension and establish Step 1 and Step 2.1 of the proof of Theorem 1.1. In $\$ 4$ we prepare some basics of geometric measure theory and establish Step 2.2. We establish Step 3 and complete the proof of Theorem 1.1 in \$5. We study a concrete example in 86 . Although the result in 96 is not used in the proof of Theorem 1.1, hopefully it will help readers to understand various concepts in the paper.

This paper is rather lengthy. We would like to suggest readers how to read it. \$5 is technically hard. So it may be reasonable to concentrate on \$3 and \$4 at the first reading. \$2 is a preparation for these two sections. So, after reading only the main definitions in $\$ 2$ (topological/metric mean dimensions, mutual information and rate distortion function), readers may skip to $₫ 3$ and return to $\$ 2$ when they need the results there. $\$ 6$ might help readers to improve the understanding. So it may be nice to briefly look at it in the midst of reading $\$ 3$ and $\$ 4$. 
Acknowledgment. This project was initiated at the Banff International Research Station meeting "Mean Dimension and Sofic Entropy Meet Dynamical Systems, Geometric Analysis and Information Theory" in 2017. We thank BIRS for hosting this workshop, and for providing ideal conditions for collaborations.

\section{Preliminaries}

2.1. Topological and metric mean dimensions. We review basics of topological and metric mean dimensions in this subsection Gro99, LW00]. Throughout this paper we assume that all simplicial complexes are finite (i.e. they have only finitely many faces).

Let $(\mathcal{X}, d)$ be a compact metric space. We introduce some metric invariants of $(\mathcal{X}, d)$. Take a positive number $\varepsilon$. Let $f: \mathcal{X} \rightarrow \mathcal{Y}$ be a continuous map from $\mathcal{X}$ to some topological space $\mathcal{Y}$. The map $f$ is said to be an $\varepsilon$-embedding if $\operatorname{diam} f^{-1}(y)<\varepsilon$ for every $y \in \mathcal{Y}$. We define the $\varepsilon$-width $\operatorname{dimension}_{\operatorname{Widim}_{\varepsilon}}(\mathcal{X}, d)$ as the minimum $n \geq 0$ such that there exists an $\varepsilon$-embedding $f: \mathcal{X} \rightarrow P$ from $\mathcal{X}$ to some $n$-dimensional simplicial complex $P$. The topological dimension of $\mathcal{X}$ is given by $\operatorname{dim} \mathcal{X}=\lim _{\varepsilon \rightarrow 0} \operatorname{Widim}_{\varepsilon}(\mathcal{X}, d)$.

We define the $\varepsilon$-covering number $\#(\mathcal{X}, d, \varepsilon)$ as the minimum $n \geq$ 1 such that there exists an open cover $\left\{U_{1}, \ldots, U_{n}\right\}$ of $\mathcal{X}$ satisfying $\operatorname{diam} U_{i}<\varepsilon$ for all $1 \leq i \leq n$. We also define the $\varepsilon$-separating number $\#_{\text {sep }}(\mathcal{X}, d, \varepsilon)$ as the maximum $n \geq 1$ such that there exist $x_{1}, \ldots, x_{n} \in \mathcal{X}$ satisfying $d\left(x_{i}, x_{j}\right) \geq \varepsilon$ for all $i \neq j$. For $0<\delta<\varepsilon / 2$

$$
\#_{\text {sep }}(\mathcal{X}, d, \varepsilon) \leq \#(\mathcal{X}, d, \varepsilon) \leq \#_{\text {sep }}(\mathcal{X}, d, \delta) \text {. }
$$

The upper and lower Minkowski dimensions (or box dimensions) of $(\mathcal{X}, d)$ are given by

$$
\begin{aligned}
& \overline{\operatorname{dim}}_{\mathrm{M}}(\mathcal{X}, d)=\limsup _{\varepsilon \rightarrow 0} \frac{\log \#(\mathcal{X}, d, \varepsilon)}{\log (1 / \varepsilon)}, \\
& \underline{\operatorname{dim}}_{\mathrm{M}}(\mathcal{X}, d)=\liminf _{\varepsilon \rightarrow 0} \frac{\log \#(\mathcal{X}, d, \varepsilon)}{\log (1 / \varepsilon)} .
\end{aligned}
$$

Example 2.1. Let $(V,\|\cdot\|)$ be a finite dimensional Banach space and $B_{r}(V)$ the closed $r$-ball around the origin $(r>0)$. Then for $0<\varepsilon<r$

$$
\begin{aligned}
& \operatorname{Widim}_{\varepsilon}\left(B_{r}(V),\|\cdot\|\right)=\operatorname{dim} V, \\
& \#\left(B_{r}(V),\|\cdot\|, \varepsilon\right) \geq(r / \varepsilon)^{\operatorname{dim} V} .
\end{aligned}
$$

(2.2) is due to Gromov [Gro99, §1.1.2]. See [T09, Appendix] for a simple proof. The proof of (2.3) is easy: Take the Lebesgue measure $\mu$ on $V$ normalized by $\mu\left(B_{r}(V)\right)=1$. Let $B_{r}(V)=U_{1} \cup \cdots \cup U_{n}$ with 
$\operatorname{diam} U_{i}<\varepsilon$. Pick $x_{i} \in U_{i}$. Then $B_{r}(V) \subset B_{\varepsilon}\left(x_{1}\right) \cup \cdots \cup B_{\varepsilon}\left(x_{n}\right)\left(B_{\varepsilon}\left(x_{i}\right)\right.$ is the closed $\varepsilon$-ball centered at $\left.x_{i}\right)$. It follows that

$$
1=\mu\left(B_{r}(V)\right) \leq \sum_{i=1}^{n} \mu\left(B_{\varepsilon}\left(x_{i}\right)\right)=n(\varepsilon / r)^{\operatorname{dim} V} .
$$

This shows $n \geq(r / \varepsilon)^{\operatorname{dim} V}$.

Let $(\mathcal{X}, T)$ be a dynamical system with a metric $d$. For $N \geq 1$ we define a new metric on $\mathcal{X}$ by

$$
d_{N}(x, y)=\max _{0 \leq n<N} d\left(T^{n} x, T^{n} y\right)
$$

We define the (topological) mean dimension by

$$
\operatorname{mdim}(\mathcal{X}, T)=\lim _{\varepsilon \rightarrow 0}\left(\lim _{N \rightarrow \infty} \frac{\operatorname{Widim}_{\varepsilon}\left(\mathcal{X}, d_{N}\right)}{N}\right)
$$

The limit always exists because $\operatorname{Widim}_{\varepsilon}\left(\mathcal{X}, d_{N}\right)$ is subadditive in $N$. The value of $\operatorname{mdim}(\mathcal{X}, T)$ is independent of the choice of $d$, namely it becomes a topological invariant of $(\mathcal{X}, T)$. We define the entropy at the resolution $\varepsilon>0$ by

$$
S(\mathcal{X}, T, d, \varepsilon)=\lim _{N \rightarrow \infty} \frac{\log \#\left(\mathcal{X}, d_{N}, \varepsilon\right)}{N},
$$

where the limit exists because $\log \#\left(\mathcal{X}, d_{N}, \varepsilon\right)$ is subadditive in $N$. We define the upper and lower metric mean dimensions by (1.5) in $\$ 1.2$.

The following two theorems were proved in [LW00, Theorem 4.2] and [Lin99, Theorem 4.3] respectively.

\section{Theorem 2.2.}

$$
\operatorname{mdim}(\mathcal{X}, T) \leq \underline{\operatorname{mdim}}_{\mathrm{M}}(\mathcal{X}, T, d) \leq \overline{\operatorname{mdim}}_{\mathrm{M}}(\mathcal{X}, T, d) .
$$

Theorem 2.3. If $(\mathcal{X}, T)$ has the marker property then there exists a metric $d$ on $\mathcal{X}$ compatible with the topology satisfying

$$
\underline{\operatorname{mim}}_{\mathrm{M}}(\mathcal{X}, T, d)=\operatorname{mdim}(\mathcal{X}, T) .
$$

Example 2.4. Let $\sigma:[0,1]^{\mathbb{Z}} \rightarrow[0,1]^{\mathbb{Z}}$ be the shift on the alphabet $[0,1]$ (the unit interval). We define a metric $d$ on it by $d(x, y)=$ $\sum_{n \in \mathbb{Z}} 2^{-|n|}\left|x_{n}-y_{n}\right|$. Then

$$
\operatorname{mdim}\left([0,1]^{\mathbb{Z}}, \sigma\right)=\operatorname{mdim}_{\mathrm{M}}\left([0,1]^{\mathbb{Z}}, \sigma, d\right)=1 .
$$

The only nontrivial point is the lower bound $\operatorname{mdim}\left([0,1]^{\mathbb{Z}}, \sigma\right) \geq 1$, which follows from (2.2); cf. also [LW00, Proposition 3.3]. 
2.2. Mutual information. Here we prepare some basics of mutual information [CT06, Chapter 2]. Throughout this subsection we fix a probability space $(\Omega, \mathbb{P})$ and assume that all random variables are defined on it.

Let $X$ and $Y$ be two random variables taking values in some measurable spaces $\mathcal{X}$ and $\mathcal{Y}$ respectively. We want to define their mutual information $I(X ; Y)$, which measures the amount of information shared by both $X$ and $Y$. If $\mathcal{X}$ and $\mathcal{Y}$ are finite set: 7 , then we set

$$
I(X ; Y)=H(X)+H(Y)-H(X, Y)=H(X)-H(X \mid Y),
$$

where $H(X \mid Y)$ is the conditional entropy of $X$ given $Y$. With the convention that $0 \log (0 / a)=0$ for all $a \geq 0$, we can also write this as

$$
I(X ; Y)=\sum_{x \in \mathcal{X}, y \in \mathcal{Y}} \mathbb{P}(X=x, Y=y) \log \frac{\mathbb{P}(X=x, Y=y)}{\mathbb{P}(X=x) \mathbb{P}(Y=y)}
$$

In general we proceed as follows. Take finite measurable partitions $\mathcal{P}=\left\{P_{1}, \ldots, P_{M}\right\}$ and $\mathcal{Q}=\left\{Q_{1}, \ldots, Q_{N}\right\}$ of $\mathcal{X}$ and $\mathcal{Y}$ respectively. For $x \in \mathcal{X}$ and $y \in \mathcal{Y}$ we set $\mathcal{P}(x)=P_{m}$ and $\mathcal{Q}(y)=Q_{n}$ where $x \in P_{m}$ and $y \in Q_{n}$ Then we can consider the mutual information $I(\mathcal{P} \circ X ; \mathcal{Q} \circ Y)$ defined by (2.4) because $\mathcal{P} \circ X$ and $\mathcal{Q} \circ Y$ take only finitely many values. We define $I(X ; Y)$ as the supremum of $I(\mathcal{P} \circ X ; \mathcal{Q} \circ Y)$ over all finite measurable partitions $\mathcal{P}$ and $\mathcal{Q}$ of $\mathcal{X}$ and $\mathcal{Y}$. This definition is compatible with (2.4) when $\mathcal{X}$ and $\mathcal{Y}$ are finite sets 8 .

We gather properties of mutual information required in the proof of the double variational principle (Theorem 1.1) below. They are not used in $\$ 3$. So readers may postpone to read the rest of this subsection until they come to $\$ 4$.

Lemma 2.5 (Data-Processing inequality). Let $X$ and $Y$ be random variables taking values in measurable spaces $\mathcal{X}$ and $\mathcal{Y}$. If $f: \mathcal{Y} \rightarrow \mathcal{Z}$ is a measurable map, then $I(X ; f(Y)) \leq I(X ; Y)$.

Proof. This immediately follows from the definition. A nontrivial point is that the above definition is compatible with (2.4) for discrete random variables.

Lemma 2.6. Let $\mathcal{X}$ and $\mathcal{Y}$ be finite sets and $\left(X_{n}, Y_{n}\right)$ a sequence of random variables taking values in $\mathcal{X} \times \mathcal{Y}$. If $\left(X_{n}, Y_{n}\right)$ converges to some $(X, Y)$ in law, then $I\left(X_{n} ; Y_{n}\right)$ converges to $I(X ; Y)$.

\footnotetext{
${ }^{7} \mathrm{We}$ always assume that the $\sigma$-algebra of a finite set is the largest one (the set of all subsets).

${ }^{8}$ We can show this by proving the data-processing inequality (Lemma 2.5) for the quantity defined by (2.4) in the case that $\mathcal{X}$ and $\mathcal{Y}$ are finite sets. See [CT06, Section 2.8].
} 
Proof. This follows from (2.4).

Lemma 2.7 (Subadditivity of mutual information). Let $X, Y, Z$ be random variables taking values in finite sets $\mathcal{X}, \mathcal{Y}, \mathcal{Z}$ respectively. Suppose $X$ and $Y$ are conditionally independent given $Z$, namely for every $x \in \mathcal{X}, y \in \mathcal{Y}$ and $z \in \mathcal{Z}$ with $\mathbb{P}(Z=z) \neq 0$ we have

$$
\mathbb{P}(X=x, Y=y \mid Z=z)=\mathbb{P}(X=x \mid Z=z) \mathbb{P}(Y=y \mid Z=z) .
$$

Then $I(X, Y ; Z) \leq I(X ; Z)+I(Y ; Z)$.

Proof. $I(X, Y ; Z)=H(X, Y)-H(X, Y \mid Z)$. From the conditional independence $H(X, Y \mid Z)=H(X \mid Z)+H(Y \mid Z)$. Hence

$$
\begin{aligned}
I(X, Y ; Z) & =H(X, Y)-H(X \mid Z)-H(Y \mid Z) \\
& \leq H(X)+H(Y)-H(X \mid Z)-H(Y \mid Z) \\
& =I(X ; Z)+I(Y ; Z) .
\end{aligned}
$$

Here we have used $H(X, Y) \leq H(X)+H(Y)$.

Let $X$ and $Y$ be random variables taking values in finite sets $\mathcal{X}$ and $\mathcal{Y}$. We set $\mu(x)=\mathbb{P}(X=x)$ and $\nu(y \mid x)=\mathbb{P}(Y=y \mid X=x)$ for $x \in \mathcal{X}$ and $y \in \mathcal{Y}$. (The conditional probability mass function $\nu(y \mid x)$ is defined only for $x \in \mathcal{X}$ with $\mathbb{P}(X=x) \neq 0$.) The mutual information $I(X ; Y)$ is determined by the distribution of $(X, Y)$, which is given by $\mu(x) \nu(y \mid x)$. It will be convenient for us to write $I(X ; Y)$ sometimes as $I(\mu, \nu)$.

Lemma 2.8 (Concavity/convexity of mutual information). In the above setting $I(\mu, \nu)$ is a concave function of $\mu(x)$ for fixed $\nu(y \mid x)$ and a convex function of $\nu(y \mid x)$ for fixed $\mu(x)$. Namely for $0 \leq t \leq 1$

$$
\begin{aligned}
I\left((1-t) \mu_{1}+t \mu_{2}, \nu\right) & \geq(1-t) I\left(\mu_{1}, \nu\right)+t I\left(\mu_{2}, \nu\right), \\
I\left(\mu,(1-t) \nu_{1}+t \nu_{2}\right) & \leq(1-t) I\left(\mu, \nu_{1}\right)+t I\left(\mu, \nu_{2}\right) .
\end{aligned}
$$

Proof. See [CT06, Theorem 2.7.4] for the detailed proof. First we prove the concavity.

$$
I(\mu, \nu)=I(X ; Y)=H(Y)-H(Y \mid X) .
$$

$H(Y)$ is a concave function of $\mu(x)$ for fixed $\nu(y \mid x)$ (since the Shannon entropy is a concave function of distribution) and $H(Y \mid X)$ is a linear function of $\mu(x)$. So $I(\mu, \nu)$ is a concave function of $\mu(x)$.

Next we prove the convexity. From the convexity of $\phi(t):=t \log t$

$$
\phi\left(\frac{a+a^{\prime}}{b+b^{\prime}}\right) \leq \frac{b}{b+b^{\prime}} \phi\left(\frac{a}{b}\right)+\frac{b^{\prime}}{b+b^{\prime}} \phi\left(\frac{a^{\prime}}{b^{\prime}}\right)
$$


for positive $a, a^{\prime}, b, b^{\prime}$. This leads to the log sum inequality:

$$
\left(a+a^{\prime}\right) \log \frac{a+a^{\prime}}{b+b^{\prime}} \leq a \log \frac{a}{b}+a^{\prime} \log \frac{a^{\prime}}{b^{\prime}} .
$$

Set $\sigma_{i}(y)=\sum_{x \in \mathcal{X}} \mu(x) \nu_{i}(y \mid x)$ for $i=1,2$.

$$
\begin{aligned}
I\left(\mu,(1-t) \nu_{1}+t \nu_{2}\right)=\sum_{x, y} & \left\{(1-t) \mu(x) \nu_{1}(y \mid x)+t \mu(x) \nu_{2}(y \mid x)\right\} \\
& \times \log \frac{(1-t) \mu(x) \nu_{1}(y \mid x)+t \mu(x) \nu_{2}(y \mid x)}{(1-t) \mu(x) \sigma_{1}(y)+t \mu(x) \sigma_{2}(y)} .
\end{aligned}
$$

Apply (2.6) to each summand: This is bounded by

$$
\sum_{x, y}(1-t) \mu(x) \nu_{1}(y \mid x) \log \frac{\mu(x) \nu_{1}(y \mid x)}{\mu(x) \sigma_{1}(y)}+\sum_{x, y} t \mu(x) \nu_{2}(y \mid x) \log \frac{\mu(x) \nu_{2}(y \mid x)}{\mu(x) \sigma_{2}(y)}
$$

which is equal to $(1-t) I\left(\mu, \nu_{1}\right)+t I\left(\mu, \nu_{2}\right)$.

We borrow the next lemma from [KD94, Lemma A.1]. This is a duality of convex programming. (See Section 2.5 of [B71], specifically [B71, Theorem 2.5.3], for further information.) Recall that the base of the logarithm is two and the natural logarithm is written as $\ln (\cdot)$.

Lemma 2.9. Let $\mathcal{X}$ and $\mathcal{Y}$ be compact metric spaces and $\rho: \mathcal{X} \times \mathcal{Y} \rightarrow$ $[0, \infty)$ a continuous function. Let $\mu$ be a Borel probability measure on $\mathcal{X}, \varepsilon>0$ and $a \geq 0$ real numbers. Suppose a continuous function 9 $\lambda: \mathcal{X} \rightarrow[0, \infty)$ satisfies

$$
\forall y \in \mathcal{Y}: \quad \int_{\mathcal{X}} \lambda(x) 2^{-a \rho(x, y)} d \mu(x) \leq 1
$$

If $X$ and $Y$ are random variables taking values in $\mathcal{X}$ and $\mathcal{Y}$ respectively and satisfying $\operatorname{Law}(X)=\mu$ and $\mathbb{E} \rho(X, Y)<\varepsilon$ then

$$
I(X ; Y) \geq-a \varepsilon+\int_{\mathcal{X}} \log \lambda(x) d \mu(x) .
$$

Proof. We divide the proof into two steps.

Step 1: Assume $\mathcal{X}$ and $\mathcal{Y}$ are finite sets. Let $\nu=\operatorname{Law}(Y)$ be the distribution of $Y$. We define a function $f(x, y)$ by $\mathbb{P}(X=x, Y=$ $y)=f(x, y) \mathbb{P}(X=x) \mathbb{P}(Y=y)$. (We do not need to define the value

\footnotetext{
${ }^{9}$ The continuity of $\rho$ and $\lambda$ is inessential. But we assume it for simplicity. Indeed in our applications, $\mathcal{X}=\mathcal{Y}, \rho$ is a distance function and $\lambda$ is a constant.
} 
$f(x, y)$ if $\mathbb{P}(X=x) \mathbb{P}(Y=y)=0$.) It follows from (2.5) that

$$
\begin{aligned}
I(X ; Y) & =\sum_{x \in \mathcal{X}, y \in \mathcal{Y}} \mathbb{P}(X=x) \mathbb{P}(Y=y) f(x, y) \log f(x, y) \\
& =\int_{\mathcal{X} \times \mathcal{Y}} f(x, y) \log f(x, y) d \mu(x) d \nu(y) .
\end{aligned}
$$

Set $g(x, y)=\lambda(x) 2^{-a \rho(x, y)}$. The right-hand side of (2.8) is equal to $-a \varepsilon+\int_{\mathcal{X} \times \mathcal{Y}} \log \lambda d \operatorname{Law}(X, Y)=-a \varepsilon+\int_{\mathcal{X} \times \mathcal{Y}} f(x, y) \log \lambda(x) d \mu(x) d \nu(y)$.

Since $-\varepsilon<-\mathbb{E} \rho(X, Y)=-\int_{\mathcal{X} \times \mathcal{Y}} \rho(x, y) f(x, y) d \mu(x) d \nu(y)$, this is less than

$$
\begin{gathered}
\int_{\mathcal{X} \times \mathcal{Y}} f(x, y) \log g(x, y) d \mu(x) d \nu(y) . \\
I(X ; Y)-\int_{\mathcal{X} \times \mathcal{Y}} f(x, y) \log g(x, y) d \mu(x) d \nu(y)=\int_{\mathcal{X} \times \mathcal{Y}} f \log (f / g) d \mu d \nu .
\end{gathered}
$$

As $\ln 2 \cdot \log (1 / u)=\ln (1 / u) \geq 1-u$, we have $\ln 2 \cdot f \log (f / g) \geq f(1-$ $g / f)=f-g$ and hence

$$
\begin{aligned}
\ln 2 \cdot \int_{\mathcal{X} \times \mathcal{Y}} f \log (f / g) d \mu d \nu & \geq \int_{\mathcal{X} \times \mathcal{Y}}(f-g) d \mu d \nu \\
& =1-\int_{\mathcal{Y}}\left(\int_{\mathcal{X}} g(x, y) d \mu(x)\right) d \nu(y) \geq 0 .
\end{aligned}
$$

Here we have used the assumption (2.7) in the last inequality.

Step 2: General case. Let $\delta>0$. Take finite partitions $\mathcal{P}=$ $\left\{P_{1}, \ldots, P_{M}\right\}$ and $\mathcal{Q}=\left\{Q_{1}, \ldots, Q_{N}\right\}$ of $\mathcal{X}$ and $\mathcal{Y}$ respectively. For each $P_{m}$ we take a point $x_{m} \in P_{m}$ satisfying $\lambda\left(x_{m}\right) \geq(1+\delta)^{-1} \sup _{P_{m}} \lambda$. We pick arbitrary $y_{n} \in Q_{n}$ for each $Q_{n}$. We set $\mathcal{X}^{\prime}=\left\{x_{1}, \ldots, x_{M}\right\}$ and $\mathcal{Y}^{\prime}=\left\{y_{1}, \ldots, y_{N}\right\}$ and define maps $\mathcal{P}: \mathcal{X} \rightarrow \mathcal{X}^{\prime}$ and $\mathcal{Q}: \mathcal{Y} \rightarrow \mathcal{Y}^{\prime}$ by $\mathcal{P}\left(P_{m}\right)=\left\{x_{m}\right\}$ and $\mathcal{Q}\left(Q_{n}\right)=\left\{y_{n}\right\}$. Set $X^{\prime}=\mathcal{P} \circ X, Y^{\prime}=\mathcal{Q} \circ Y$ and $\mu^{\prime}=\mathcal{P}_{*} \mu=\operatorname{Law}\left(X^{\prime}\right)$.

From the continuity of $\rho$ and $\lambda$, by taking $\mathcal{P}$ and $\mathcal{Q}$ sufficiently fine, we can assume $\mathbb{E} \rho\left(X^{\prime}, Y^{\prime}\right)<\varepsilon$ and

$$
\forall y \in \mathcal{Y}^{\prime}, \quad \int_{\mathcal{X}^{\prime}} \lambda(x) 2^{-a \rho(x, y)} d \mu^{\prime}(x) \leq 1+\delta .
$$

Then we can apply Step 1 to $X^{\prime}, Y^{\prime}$ and the function $\lambda^{\prime}\left(x_{m}\right):=(1+$ $\delta)^{-1} \lambda\left(x_{m}\right)$. This yields

$$
I\left(X^{\prime} ; Y^{\prime}\right) \geq-a \varepsilon+\int_{\mathcal{X}^{\prime}} \log \lambda^{\prime} d \mu^{\prime}=-a \varepsilon-\log (1+\delta)+\int_{\mathcal{X}^{\prime}} \log \lambda d \mu^{\prime} .
$$


It follows from the choice of $x_{m}$ that

$$
\int_{\mathcal{X}^{\prime}} \log \lambda d \mu^{\prime} \geq-\log (1+\delta)+\int_{\mathcal{X}} \log \lambda d \mu
$$

As $I(X ; Y) \geq I\left(X^{\prime} ; Y^{\prime}\right)$ by the definition of mutual information,

$$
I(X ; Y) \geq-a \varepsilon-2 \log (1+\delta)+\int_{\mathcal{X}} \log \lambda d \mu .
$$

Let $\delta \rightarrow 0$. This shows the statement.

The next lemma is essentially due to [KD94, Proposition 3.2]. This is a key to connect geometric measure theory to rate distortion theory.

Lemma 2.10. Let $\varepsilon$ and $\delta$ be positive numbers with $2 \varepsilon \log (1 / \varepsilon) \leq \delta$. Let $0 \leq \tau \leq \min (\varepsilon / 3, \delta / 2)$ and $s \geq 0$ be real numbers. Let $(\mathcal{X}, d)$ be a compact metric space with a Borel probability measure $\mu$ satisfying

$$
\mu(E) \leq(\tau+\operatorname{diam} E)^{s}, \quad \forall E \subset \mathcal{X} \text { with } \operatorname{diam} E<\delta .
$$

Let $X$ and $Y$ be random variables taking values in $\mathcal{X}$ with $\operatorname{Law}(X)=\mu$ and $\mathbb{E} d(X, Y)<\varepsilon$. Then

$$
I(X ; Y) \geq s \log (1 / \varepsilon)-C(s+1)
$$

where $C$ is a universal positive constant independent of $\varepsilon, \delta, \tau, s,(\mathcal{X}, d), \mu$.

Proof. We apply Lemma 2.9 with $a=s / \varepsilon$. Set $b=a \ln 2$ and estimate $\int_{\mathcal{X}} 2^{-a d(x, y)} d \mu(x)=\int_{\mathcal{X}} e^{-b d(x, y)} d \mu(x)$ for each $y \in \mathcal{X}$ :

$$
\begin{aligned}
\int_{\mathcal{X}} e^{-b d(x, y)} d \mu(x) & =\int_{0}^{1} \mu\left\{x \mid e^{-b d(x, y)} \geq u\right\} d u \\
& =\int_{0}^{\infty} \mu\{x \mid d(x, y) \leq v\} b e^{-b v} d v \quad\left(\text { set } u=e^{-b v}\right) \\
& =\left(\int_{0}^{\tau}+\int_{\tau}^{\delta / 2}+\int_{\delta / 2}^{\infty}\right) \mu\{x \mid d(x, y) \leq v\} b e^{-b v} d v
\end{aligned}
$$

In the last line we have used $\tau \leq \delta / 2$. From (2.9)

$$
\int_{0}^{\tau} \mu\{x \mid d(x, y) \leq v\} b e^{-b v} d v \leq(3 \tau)^{s} \int_{0}^{\infty} b e^{-b v} d v=(3 \tau)^{s} \leq \varepsilon^{s}
$$


where we have used $\tau \leq \varepsilon / 3$.

$$
\begin{aligned}
\int_{\tau}^{\delta / 2} \mu\{x \mid d(x, y) \leq v\} b e^{-b v} d v & \leq \int_{\tau}^{\delta / 2}(\tau+2 v)^{s} b e^{-b v} d v \\
& \leq 3^{s} \int_{\tau}^{\delta / 2} v^{s} b e^{-b v} d v \\
& \leq(3 / b)^{s} \int_{0}^{\infty} t^{s} e^{-t} d t \quad(\text { set } t=b v) \\
& =\varepsilon^{s}(3 \log e)^{s} s^{-s} \Gamma(s+1) .
\end{aligned}
$$

In the last step we have used $b=s \ln 2 / \varepsilon=s /(\varepsilon \log e)$.

$$
\begin{aligned}
\int_{\delta / 2}^{\infty} \mu\{x \mid d(x, y) \leq v\} b e^{-b v} d v & \leq \int_{\delta / 2}^{\infty} b e^{-b v} d v \\
& =e^{-b \delta / 2}=\left(2^{-\delta /(2 \varepsilon)}\right)^{s} \leq \varepsilon^{s} .
\end{aligned}
$$

In the last inequality we have used $2 \varepsilon \log (1 / \varepsilon) \leq \delta$. Summing the above estimates, we get

$$
\int_{\mathcal{X}} 2^{-a d(x, y)} d \mu(x) \leq \varepsilon^{s}\left\{2+(3 \log e)^{s} s^{-s} \Gamma(s+1)\right\} .
$$

Thus the constant function $\lambda(x):=\varepsilon^{-s}\left\{2+(3 \log e)^{s} s^{-s} \Gamma(s+1)\right\}^{-1}$ satisfies

From Lemma 2.9

$$
\forall y \in \mathcal{X}: \quad \int_{\mathcal{X}} \lambda(x) 2^{-a d(x, y)} d \mu(x) \leq 1
$$

$$
\begin{aligned}
I(X ; Y) & \geq-a \varepsilon+\int_{\mathcal{X}} \log \lambda d \mu \\
& =s \log (1 / \varepsilon)-s-\log \left\{2+(3 \log e)^{s} s^{-s} \Gamma(s+1)\right\} .
\end{aligned}
$$

Recalling Stirling's formula $\Gamma(s+1) \sim s^{s} e^{-s} \sqrt{2 \pi s}$, we can find a universal constant $C>0$ satisfying

$$
s+\log \left\{2+(3 \log e)^{s} s^{-s} \Gamma(s+1)\right\} \leq C+C s .
$$

This proves the statement.

2.3. Rate distortion theory. Here we review rate distortion function ([Sh48, Sh59], [CT06, Chapter 10]). The Shannon entropy is the fundamental limit in lossless data compression of discrete random variables and processes. For a stationary stochastic process $X_{1}, X_{2}, \ldots$, its entropy is equal to the minimum expected number of bits per symbol for describing the process. But if random variables $X_{n}$ take continuously many values, the entropy is simply infinite (namely, we cannot describe continuous variables perfectly within finitely many bits). For 
continuous random variables and processes (e.g. audio signals, images, etc.) we have to consider lossy data compression method 10 achieving some distortion constraint. This is the primary object of rate distortion theory. Rate distortion function is the fundamental limit of data compression in this context.

Let $(\mathcal{X}, T)$ be a dynamical system with a metric $d$ and an invariant probability measure $\mu$. For $\varepsilon>0$ we define the rate distortion function $R(d, \mu, \varepsilon)$ as the infimum of

$$
\frac{I(X ; Y)}{N}
$$

where $N>0$ is a natural number, $X$ and $Y=\left(Y_{0}, \ldots, Y_{N-1}\right)$ are random variables defined on some probability space $(\Omega, \mathbb{P})$ such that all $X$ and $Y_{n}$ take values in $\mathcal{X}$ and satisfy

$$
\operatorname{Law}(X)=\mu, \quad \mathbb{E}\left(\frac{1}{N} \sum_{n=0}^{N-1} d\left(T^{n} X, Y_{n}\right)\right)<\varepsilon .
$$

We define the lower and upper rate distortion dimensions by (1.2) in $\$ 1.1$

Remark 2.11. In the above definition of rate distortion function we can assume that $Y$ takes only finitely many values, namely its distribution is supported on a finite set: Take a finite partition $\mathcal{P}$ of $\mathcal{X}$ and pick a point $x_{P} \in P$ for each $P \in \mathcal{P}$. Define $f: \mathcal{X} \rightarrow \mathcal{X}$ by $f(P)=\left\{x_{P}\right\}$ for $P \in \mathcal{P}$ and set $Z=\left(Z_{0}, \ldots, Z_{N-1}\right)=\left(f\left(Y_{0}\right), \ldots, f\left(Y_{N-1}\right)\right)$. If $\mathcal{P}$ is sufficiently fine then

$$
\mathbb{E}\left(\frac{1}{N} \sum_{n=0}^{N-1} d\left(T^{n} X, Z_{n}\right)\right)<\varepsilon .
$$

From the definition of mutual information (or the data-processing inequality; Lemma 2.5),

$$
I(X ; Z) \leq I(X ; Y) .
$$

The random variable $Z$ takes only finitely many values.

The rate distortion function $R(d, \mu, \varepsilon)$ is the minimum rate when we try to quantize the process $\left\{T^{n} X\right\}_{n \in \mathbb{Z}}$ within the average distortion bound by $\varepsilon$ [Gra90, Chapter 11]: For simplicity 11 , suppose $\mu$ is

\footnotetext{
${ }^{10}$ E.g. expanding signals in a wavelet basis, discarding small terms and quantizing the remaining terms.

${ }^{11}$ Although the "operational meaning" of rate distortion function is important for the understanding, we do not use it in the paper. So we do not give a complete explanation. See LDN79, ECG94, Gra90, for the non-ergodic case.
} 
ergodic. For any $\delta>0$, if $N$ is sufficiently large, there exists a map $f=\left(f_{0}, \ldots, f_{N-1}\right): \mathcal{X} \rightarrow \mathcal{X}^{N}$ which has a finite range (i.e. it takes only finitely many values) and satisfies

$$
\frac{\log |f(\mathcal{X})|}{N}<R(d, \mu, \varepsilon)+\delta, \quad \mathbb{E}\left(\frac{1}{N} \sum_{n=0}^{N-1} d\left(T^{n} X, f_{n}(X)\right)\right)<\varepsilon .
$$

Namely we can approximate the process $X, T X, \ldots, T^{N-1} X$ by the quantization $f_{0}(X), f_{1}(X), \ldots, f_{N-1}(X)$ within the average distortion bound by $\varepsilon$. The bits per iterate required for this description is less than $R(d, \mu, \varepsilon)+\delta$.

Example 2.12. Consider the shift $\sigma:[0,1]^{\mathbb{Z}} \rightarrow[0,1]^{\mathbb{Z}}$ with a metric $d(x, y)=\sum_{n \in \mathbb{Z}} 2^{-|n|}\left|x_{n}-y_{n}\right|$ and an invariant probability measure

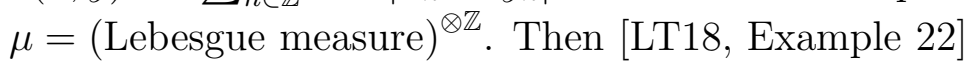

$$
\operatorname{rdim}\left([0,1]^{\mathbb{Z}}, \sigma, d, \mu\right)=1 \text {. }
$$

\section{Mean Hausdorff dimension and the proof of the DOUBLE VARIATIONAL PRINCIPLE}

In this section we introduce the key concept of the paper - mean Hausdorff dimension. We develop various comparison estimates between topological/metric mean dimensions, mean Hausdorff dimension and rate distortion dimension. Some of the proofs are postponed to later sections. We prove the double variational principle (Theorem 1.1) by using these comparison estimates at the end of $\$ 3.2$

3.1. Definition of mean Hausdorff dimension. Let $(\mathcal{X}, d)$ be a compact metric space. For $s \geq 0$ and $\varepsilon>0$ we define $\mathcal{H}_{\varepsilon}^{s}(\mathcal{X}, d)$ as

$$
\inf \left\{\sum_{n=1}^{\infty}\left(\operatorname{diam} E_{n}\right)^{s} \mid \mathcal{X}=\bigcup_{n=1}^{\infty} E_{n} \text { with diam } E_{n}<\varepsilon \text { for all } n \geq 1\right\} \text {. }
$$

Here we use the convention that $0^{0}=1$ and $\operatorname{diam}(\emptyset)^{s}=0$. We also define

$$
\mathcal{H}_{\infty}^{s}(\mathcal{X}, d)=\inf \left\{\sum_{n=1}^{\infty}\left(\operatorname{diam} E_{n}\right)^{s} \mid \mathcal{X}=\bigcup_{n=1}^{\infty} E_{n}\right\}
$$

We set

$$
\operatorname{dim}_{\mathrm{H}}(\mathcal{X}, d, \varepsilon)=\sup \left\{s \geq 0 \mid \mathcal{H}_{\varepsilon}^{s}(\mathcal{X}, d) \geq 1\right\} .
$$

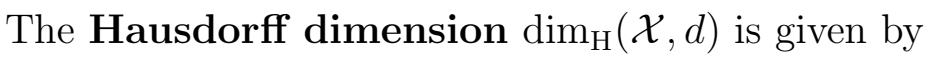

$$
\operatorname{dim}_{\mathrm{H}}(\mathcal{X}, d)=\lim _{\varepsilon \rightarrow 0} \operatorname{dim}_{\mathrm{H}}(\mathcal{X}, d, \varepsilon) .
$$


Let $(\mathcal{X}, T)$ be a dynamical system with a metric $d$. As in $\$ 2.1$ we set $d_{N}(x, y)=\max _{0 \leq n<N} d\left(T^{n} x, T^{n} y\right)$. We define the mean Hausdorff dimension by

$$
\operatorname{mdim}_{\mathrm{H}}(\mathcal{X}, T, d)=\lim _{\varepsilon \rightarrow 0}\left(\limsup _{N \rightarrow \infty} \frac{1}{N} \operatorname{dim}_{\mathrm{H}}\left(\mathcal{X}, d_{N}, \varepsilon\right)\right) .
$$

Remark 3.1. We can also define the lower mean Hausdorff dimension $\underline{\operatorname{mdim}}_{\mathrm{H}}(\mathcal{X}, T, d)$ by replacing $\lim \sup _{N}$ in (3.1) with $\lim \inf _{N}$. But we do not seriously use this concept in the paper.

3.2. Comparison between various dynamical dimensions. The following proposition extends Theorem 2.2 to mean Hausdorff dimension and rate distortion dimension.

Proposition 3.2. Let $(\mathcal{X}, T)$ be a dynamical system with a metric d and an invariant probability measure $\mu$.

$$
\begin{gathered}
\operatorname{mdim}(\mathcal{X}, T) \leq \operatorname{mdim}_{\mathrm{H}}(\mathcal{X}, T, d) \leq \underline{\operatorname{mdim}}_{\mathrm{M}}(\mathcal{X}, T, d) \\
\overline{\operatorname{rdim}}(\mathcal{X}, T, d, \mu) \leq \overline{\operatorname{mdim}}_{\mathrm{M}}(\mathcal{X}, T, d) \\
\underline{\operatorname{rdim}}(\mathcal{X}, T, d, \mu) \leq \underline{\operatorname{mdim}}_{\mathrm{M}}(\mathcal{X}, T, d) .
\end{gathered}
$$

Proof. The nontrivial result is only $\operatorname{mdim}(\mathcal{X}, T) \leq \operatorname{mdim}_{\mathrm{H}}(\mathcal{X}, T, d)$. The rest of the statement is easy. We first prove easy estimates. Let $N \geq 1$ and $\varepsilon>0$. Consider an open cover $\mathcal{X}=U_{1} \cup \cdots \cup U_{n}$ with $\operatorname{diam}\left(U_{i}, d_{N}\right)<\varepsilon$ and $n=\#\left(\mathcal{X}, d_{N}, \varepsilon\right)$.

We have $\mathcal{H}_{\varepsilon}^{s}\left(\mathcal{X}, d_{N}\right) \leq n \varepsilon^{s}$. If $s>\log n / \log (1 / \varepsilon)$ then $\mathcal{H}^{s}\left(\mathcal{X}, d_{N}\right)<$ 1. This shows

$$
\operatorname{dim}_{H}\left(\mathcal{X}, d_{N}, \varepsilon\right) \leq \frac{\log \#\left(\mathcal{X}, d_{N}, \varepsilon\right)}{\log (1 / \varepsilon)}
$$

Divide this by $N$ and take limits with respect to $N$ and then $\varepsilon$. It follows that $\operatorname{mim}_{\mathrm{H}}(\mathcal{X}, T, d) \leq \underline{\operatorname{mdim}}_{\mathrm{M}}(\mathcal{X}, T, d)$.

Next we consider (3.3). Let $X$ be a random variable obeying $\mu$. Choose a point $x_{i}$ from each $U_{i}$. We define $f: \mathcal{X} \rightarrow\left\{x_{1}, \ldots, x_{n}\right\}$ by $f(x)=x_{i}$ where $i$ is the smallest number with $x \in U_{i}$. Set $Y=$ $\left(f(X), T f(X), \ldots, T^{N-1} f(X)\right)$. Since $d\left(T^{k} x, T^{k} f(x)\right)<\varepsilon$ for all $x \in \mathcal{X}$ and $0 \leq k<N$,

$$
\frac{1}{N} \sum_{k=0}^{N-1} d\left(T^{k} X, T^{k} f(X)\right)<\varepsilon .
$$

Since $Y$ takes at most $n=\#\left(\mathcal{X}, d_{N}, \varepsilon\right)$ values,

$$
I(X ; Y) \leq H(Y) \leq \log n .
$$


This shows

$$
R(d, \mu, \varepsilon) \leq \frac{\log \#\left(\mathcal{X}, d_{N}, \varepsilon\right)}{N}
$$

Letting $N \rightarrow \infty$, we get $R(d, \mu, \varepsilon) \leq S(\mathcal{X}, T, d, \varepsilon)$. Divide this by $\log (1 / \varepsilon)$ and take the upper/lower limits with respect to $\varepsilon$. This proves (3.3).

Now we come to the main point; the comparison between mean dimension and mean Hausdorff dimension. We use the idea of the proof of Theorem 2.2 (comparison between topological/metric mean dimensions). We need some preliminary claims. In the sequel we denote by $\nu_{N}$ and $\|\cdot\|_{\infty}$ the standard Lebesgue measure and $\ell^{\infty}$-norm on $\mathbb{R}^{N}$. For $A \subset\{1,2, \ldots, N\}$ we denote by $\pi_{A}: \mathbb{R}^{N} \rightarrow \mathbb{R}^{A}$ the projection to the $A$ coordinates. For $0 \leq n \leq N$ we define $P_{n}$ as the $n$-skeleton of the cube $[0,1]^{N}$, i.e. the set of $x \in[0,1]^{N}$ satisfying $\mid\left\{k \mid x_{k}=0\right.$ or 1$\} \mid \geq N-n$.

Claim 3.3. Let $K \subset[0,1]^{N}$ be a closed subset and $1 \leq n \leq N$.

(1) $\nu_{N}(K) \leq 2^{N} \mathcal{H}_{\infty}^{N}\left(K,\|\cdot\|_{\infty}\right)$.

(2) $\nu_{N}\left(\bigcup_{|A| \geq n} \pi_{A}^{-1}\left(\pi_{A} K\right)\right) \leq 4^{N} \mathcal{H}_{\infty}^{n}\left(K,\|\cdot\|_{\infty}\right)$.

(3) If $\mathcal{H}_{\infty}^{n+1}\left(K,\|\cdot\|_{\infty}\right)<4^{-N}$ then there exists a 1-embedding $f$ : $K \rightarrow P_{n}$, i.e. a continuous map satisfying $f(x) \neq f(y)$ for any $x, y \in K$ with $\|x-y\|_{\infty}=1$.

Proof. (1) Let $K=\bigcup_{k \geq 1} E_{k}$ and set $l_{k}=\operatorname{diam}\left(E_{k},\|\cdot\|_{\infty}\right)$. Take $x_{k} \in$ $E_{k}$. Since $E_{k} \subset x_{k}+\left[-l_{k}, l_{k}\right]^{N}$,

$$
\nu_{N}(K) \leq \sum_{k=1}^{\infty}\left(2 l_{k}\right)^{N}=2^{N} \sum_{k=1}^{\infty} l_{k}^{N}
$$

(2) $\nu_{N}\left(\bigcup_{|A| \geq n} \pi_{A}^{-1}\left(\pi_{A} K\right)\right)$ is bounded by

$$
\sum_{|A| \geq n} \nu_{N}\left(\pi_{A}^{-1}\left(\pi_{A} K\right)\right)=\sum_{|A| \geq n} \nu_{|A|}\left(\pi_{A} K\right)
$$

Apply the above $(1)$ to $\pi_{A} K \subset[0,1]^{A}$ :

$$
\nu_{|A|}\left(\pi_{A} K\right) \leq 2^{|A|} \mathcal{H}_{\infty}^{|A|}\left(\pi_{A} K,\|\cdot\|_{\infty}\right) \leq 2^{N} \mathcal{H}_{\infty}^{|A|}\left(\pi_{A} K,\|\cdot\|_{\infty}\right)
$$


Since $\pi_{A}$ is one-Lipschitz, $\mathcal{H}_{\infty}^{|A|}\left(\pi_{A} K,\|\cdot\|_{\infty}\right) \leq \mathcal{H}_{\infty}^{|A|}\left(K,\|\cdot\|_{\infty}\right)$. Thus

$$
\begin{aligned}
\nu_{N}\left(\bigcup_{|A| \geq n} \pi_{A}^{-1}\left(\pi_{A} K\right)\right) & \leq 2^{N} \sum_{|A| \geq n} \mathcal{H}_{\infty}^{|A|}\left(K,\|\cdot\|_{\infty}\right) \\
& \leq 2^{N} \sum_{|A| \geq n} \mathcal{H}_{\infty}^{n}\left(K,\|\cdot\|_{\infty}\right) \\
& \leq 4^{N} \mathcal{H}_{\infty}^{n}\left(K,\|\cdot\|_{\infty}\right) .
\end{aligned}
$$

(3) If $n=N$ then the statement is trivial. So we assume $n<N$. It follows from the above (2) that

$$
\nu_{N}\left(\bigcup_{|A| \geq n+1} \pi_{A}^{-1}\left(\pi_{A} K\right)\right)<1
$$

In particular we can find $q \in(0,1)^{N}$ outside of $\bigcup_{|A| \geq n+1} \pi_{A}^{-1}\left(\pi_{A} K\right)$. For $1 \leq m \leq N$ we set

$$
C_{m}=P_{m} \cap \bigcup_{|A|=m} \pi_{A}^{-1}\left(\pi_{A}(q)\right)
$$

This is a finite set. (Each facet of $P_{m}$ contains exactly one point of $C_{m}$.) By using the central projection from each point of $C_{m}$, we define a continuous map $g_{m}: P_{m} \backslash C_{m} \rightarrow P_{m-1}$. This map has the following properties:

- $\left\|g_{m}(x)-g_{m}(y)\right\|_{\infty}=1$ for $x, y \in P_{m} \backslash C_{m}$ with $\|x-y\|_{\infty}=1$.

- For $1 \leq l<m$

$$
g_{m}\left(P_{m} \backslash \bigcup_{|A|=l} \pi_{A}^{-1}\left(\pi_{A}(q)\right)\right)=P_{m-1} \backslash \bigcup_{|A|=l} \pi_{A}^{-1}\left(\pi_{A}(q)\right) .
$$

Since $K \cap \bigcup_{|A| \geq n+1} \pi_{A}^{-1}\left(\pi_{A}(q)\right)=\emptyset$, we can define $f=g_{n+1} \circ g_{n+2} \circ \cdots \circ$ $g_{N}: K \rightarrow P_{n}$. If $x, y \in K$ satisfy $\|x-y\|_{\infty}=1$ then $\|f(x)-f(y)\|_{\infty}=$ 1. In particular $f$ is a 1-embedding.

Claim 3.4. Let $N$ be a positive integer and $\varepsilon, \delta, s, \tau, L$ positive numbers with $4^{N} L^{s+\tau} \delta^{\tau}<1$. Let $(K, d)$ be a compact metric space with $\operatorname{dim}_{\mathrm{H}}(K, d, \delta)<s$. Suppose there exists an L-Lipschitz map $\varphi:(K, d) \rightarrow$ $\left([0,1]^{N},\|\cdot\|_{\infty}\right)$ such that if $x, y \in K$ satisfy $\|\varphi(x)-\varphi(y)\|_{\infty}<1$ then $d(x, y)<\varepsilon$. Then $\operatorname{Widim}_{\varepsilon}(K, d) \leq s+\tau$. 
Proof. It follows from $\operatorname{dim}_{\mathrm{H}}(K, d, \delta)<s$ that there exists a covering $K=\bigcup_{n=1}^{\infty} E_{n}$ satisfying $\operatorname{diam} E_{n}<\delta$ and $\sum_{n=1}^{\infty}\left(\operatorname{diam} E_{n}\right)^{s}<1$. Then

$$
\mathcal{H}_{\infty}^{s+\tau}(K, d) \leq \sum_{n=1}^{\infty}\left(\operatorname{diam} E_{n}\right)^{s+\tau}<\delta^{\tau} .
$$

Since $\varphi$ is $L$-Lipschitz,

$$
\mathcal{H}_{\infty}^{s+\tau}\left(\varphi(K),\|\cdot\|_{\infty}\right)<L^{s+\tau} \delta^{\tau}<4^{-N}
$$

Hence $\mathcal{H}_{\infty}^{\lfloor s+\tau\rfloor+1}\left(\varphi(K),\|\cdot\|_{\infty}\right)<4^{-N}$. Apply Claim 3.3 (3) to $\varphi(K)$ : There exists a 1-embedding $f: \varphi(K) \rightarrow P_{\lfloor s+\tau\rfloor}$. Then $f \circ \varphi: K \rightarrow$ $P_{\lfloor s+\tau\rfloor}$ becomes an $\varepsilon$-embedding. The skeleton $P_{\lfloor s+\tau\rfloor}$ admits a structure of a $\lfloor s+\tau\rfloor$-dimensional simplicial complex. So $\operatorname{Widim}_{\varepsilon}(K, d) \leq\lfloor s+$ $\tau\rfloor$.

We start the proof of $\operatorname{mdim}(\mathcal{X}, T) \leq \operatorname{mdim}_{\mathrm{H}}(\mathcal{X}, T, d)$. We can assume $\operatorname{mdim}_{\mathrm{H}}(\mathcal{X}, T, d)<\infty$. We take $\tau>0$ and $s>\operatorname{mdim}_{\mathrm{H}}(\mathcal{X}, T, d)$. Let $\varepsilon>0$.

Claim 3.5. There exist a positive number $L$, a positive integer $M$ and an L-Lipschitz map $\varphi:(\mathcal{X}, d) \rightarrow\left([0,1]^{M},\|\cdot\|_{\infty}\right)$ such that if $x, y \in \mathcal{X}$ satisfy $\|\varphi(x)-\varphi(y)\|_{\infty}<1$ then $d(x, y)<\varepsilon$.

Proof. Choose a Lipschitz function $\psi: \mathbb{R} \rightarrow[0,1]$ satisfying $\psi(t)=1$ for $t \leq \varepsilon / 4$ and $\psi(t)=0$ for $t \geq \varepsilon / 2$. Take an $\varepsilon / 4$-spanning subset $\left\{x_{1}, \ldots, x_{M}\right\} \subset \mathcal{X}$, i.e. so that for any $x \in \mathcal{X}$ there exists $x_{i}$ with $d\left(x, x_{i}\right)<\varepsilon / 4$. We define $\varphi: \mathcal{X} \rightarrow[0,1]^{M}$ by

$$
\varphi(x)=\left(\psi\left(d\left(x, x_{1}\right)\right), \ldots, \psi\left(d\left(x, x_{M}\right)\right)\right) .
$$

For $N \geq 1$ we define an $L$-Lipschitz map

$$
\varphi_{N}:\left(\mathcal{X}, d_{N}\right) \rightarrow\left(\left([0,1]^{M}\right)^{N},\|\cdot\|_{\infty}\right)
$$

by $\varphi_{N}(x)=\left(\varphi(x), \varphi(T x), \ldots, \varphi\left(T^{N-1} x\right)\right)$. This has the property that if $x, y \in \mathcal{X}$ satisfy $\left\|\varphi_{N}(x)-\varphi_{N}(y)\right\|_{\infty}<1$ then $d_{N}(x, y)<\varepsilon$.

Choose a sufficiently small $\delta>0$ satisfying $4^{M} L^{s+\tau} \delta^{\tau}<1$. It follows from $12 \operatorname{mdim}_{\mathrm{H}}(\mathcal{X}, T, d)<s$ that there exists $N_{1}<N_{2}<N_{3}<\cdots \rightarrow \infty$ satisfying $\operatorname{dim}_{\mathrm{H}}\left(\mathcal{X}, d_{N_{k}}, \delta\right)<s N_{k}$. Note

$$
4^{M N_{k}} L^{s N_{k}+\tau N_{k}} \delta^{\tau N_{k}}=\left(4^{M} L^{s+\tau} \delta^{\tau}\right)^{N_{k}}<1 .
$$

\footnotetext{
${ }^{12}$ Indeed here we use only $\underline{\operatorname{mim}}_{\mathrm{H}}(\mathcal{X}, T, d)<s$.
} 
Then we can apply Claim 3.4 to the space $\left(\mathcal{X}, d_{N_{k}}\right)$ and the map $\varphi_{N_{k}}$ with the parameters $M N_{k}, \varepsilon, \delta, s N_{k}, \tau N_{k}, L$. This provides

$$
\operatorname{Widim}_{\varepsilon}\left(\mathcal{X}, d_{N_{k}}\right) \leq s N_{k}+\tau N_{k}
$$

and hence

$$
\lim _{N \rightarrow \infty} \frac{1}{N} \operatorname{Widim}_{\varepsilon}\left(\mathcal{X}, d_{N}\right) \leq s+\tau .
$$

The right-hand side is independent of $\varepsilon$. Thus $\operatorname{mdim}(\mathcal{X}, T) \leq s+\tau$. Let $\tau \rightarrow 0$ and $s \rightarrow \operatorname{mdim}_{\mathrm{H}}(\mathcal{X}, T, d)$. This proves the statement.

Remark 3.6. The above proof actually shows

$$
\operatorname{mdim}(\mathcal{X}, T) \leq \underline{\operatorname{mdim}}_{\mathrm{H}}(\mathcal{X}, T, d),
$$

where the right-hand side is the lower mean Hausdorff dimension (Remark 3.1).

Example 3.7. Let $\sigma:[0,1]^{\mathbb{Z}} \rightarrow[0,1]^{\mathbb{Z}}$ be the shift on the alphabet $[0,1]$ with a metric $d(x, y)=\sum_{n \in \mathbb{Z}} 2^{-|n|}\left|x_{n}-y_{n}\right|$ as in Example 2.4. Then $\operatorname{mdim}_{\mathrm{H}}\left([0,1]^{\mathbb{Z}}, \sigma, d\right)=1$ because

$$
\begin{aligned}
1 & =\operatorname{mdim}\left([0,1]^{\mathbb{Z}}, \sigma\right) \\
& \leq \operatorname{mdim}_{\mathrm{H}}\left([0,1]^{\mathbb{Z}}, \sigma, d\right) \leq \operatorname{mdim}_{\mathrm{M}}\left([0,1]^{\mathbb{Z}}, \sigma, d\right)=1 .
\end{aligned}
$$

The next two theorems are the most crucial ingredients of the proof of the double variational principle. Their proofs are postponed to later sections. Before stating the results we need to introduce a concept expressing some regularity of metrics:

Definition 3.8. Let $(\mathcal{X}, d)$ be a compact metric space. It is said to have the tame growth of covering numbers if for every $\delta>0$

$$
\lim _{\varepsilon \rightarrow 0} \varepsilon^{\delta} \log \#(\mathcal{X}, d, \varepsilon)=0 .
$$

Notice that this is purely a condition on metrics and does not involve dynamics.

Example 3.9. (1) If $\mathcal{X}$ is a compact subset of a finite dimensional Banach space $(V,\|\cdot\|)$, then $(\mathcal{X},\|\cdot\|)$ has the tame growth of covering numbers because $\#(\mathcal{X},\|\cdot\|, \varepsilon)=O\left(\varepsilon^{-\operatorname{dim} V}\right)$.

(2) If a compact metric space $(K, \rho)$ has the tame growth of covering numbers, then the following metric $d$ on the shift space $K^{\mathbb{Z}}$ also has the tame growth of covering numbers:

$$
d(x, y)=\sum_{n \in \mathbb{Z}} 2^{-|n|} \rho\left(x_{n}, y_{n}\right) .
$$

(3) It follows from (1) and (2) that the metric $d(x, y)=\sum_{n \in \mathbb{Z}} 2^{-|n|} \mid x_{n}-$ $y_{n} \mid$ on $[0,1]^{\mathbb{Z}}$ has the tame growth of covering numbers. 
The next lemma shows that the tame growth of covering numbers is a fairly mild condition.

Lemma 3.10. Let $(\mathcal{X}, d)$ be a compact metric space. There exists a metric $d^{\prime}$ on $\mathcal{X}$ such that $d^{\prime}(x, y) \leq d(x, y)$ and $\left(\mathcal{X}, d^{\prime}\right)$ has the tame growth of covering numbers. In particular every compact metrizable space admits a metric having the tame growth of covering numbers.

Proof. We can assume $\operatorname{diam}(\mathcal{X}, d) \leq 1$. Let $K=[0,1]^{\mathbb{N}}$ be the onesided infinite product of the unit interval. We define a metric $\rho$ on it by $\rho(x, y)=\sum_{i \geq 1} 2^{-i}\left|x_{i}-y_{i}\right|$. As in Example 3.9, $(K, \rho)$ has the tame growth of covering numbers. Take a countable dense subset $\left\{x_{i}\right\}_{i=1}^{\infty} \subset$ $\mathcal{X}$. We define $f: \mathcal{X} \rightarrow K$ by $f(x)=\left(d\left(x, x_{i}\right)\right)_{i=1}^{\infty} \cdot f$ is a topological embedding and it is one-Lipschitz:

$$
\rho(f(x), f(y))=\sum_{i=1}^{\infty} 2^{-i}\left|d\left(x, x_{i}\right)-d\left(y, x_{i}\right)\right| \leq \sum_{i=1}^{\infty} 2^{-i} d(x, y)=d(x, y) .
$$

The metric $d^{\prime}(x, y):=\rho(f(x), f(y))$ satisfies the requirements.

Recall that we have denoted by $\mathscr{M}^{T}(\mathcal{X})$ the set of all $T$-invariant Borel probability measures on $\mathcal{X}$.

Theorem 3.11 (Existence of nice measures). Let $(\mathcal{X}, T)$ be a dynamical system with a metric $d$ such that $(\mathcal{X}, d)$ has the tame growth of covering numbers. Then

$$
\operatorname{mdim}_{\mathrm{H}}(\mathcal{X}, T, d) \leq \sup _{\mu \in \mathscr{M}^{T}(\mathcal{X})} \underline{\operatorname{rdim}}(\mathcal{X}, T, d, \mu) .
$$

Theorem 3.12 (Existence of nice metrics). If a dynamical system $(\mathcal{X}, T)$ has the marker property, then there exists a metric $d$ on $\mathcal{X}$ compatible with the topology such that

$$
\overline{\operatorname{mdim}}_{\mathrm{M}}(\mathcal{X}, T, d)=\operatorname{mdim}(\mathcal{X}, T) .
$$

The inequalities $\operatorname{mdim}(\mathcal{X}, T) \leq \underline{\operatorname{mdim}}_{\mathrm{M}}(\mathcal{X}, T, d) \leq \overline{\operatorname{mdim}}_{\mathrm{M}}(\mathcal{X}, T, d)$ always hold true. So $\operatorname{mdim}(\mathcal{X}, T)=\overline{\operatorname{mdim}}_{\mathrm{M}}(\mathcal{X}, T, d)$ implies that $\operatorname{mdim}_{\mathrm{M}}(\mathcal{X}, T, d)$ exists and is equal to $\operatorname{mdim}(\mathcal{X}, T)$.

Corollary 3.13. Let $(\mathcal{X}, T)$ be a dynamical system with a metric d. Then

$$
\operatorname{mdim}(\mathcal{X}, T) \leq \sup _{\mu \in \mathscr{M}^{T}(\mathcal{X})} \underline{\operatorname{rdim}}(\mathcal{X}, T, d, \mu) .
$$

Proof. Notice that if $d$ has the tame growth of covering numbers then the statement immediately follows from Proposition 3.2 and Theorem 3.11. Hence the problem is how to reduce the general case to this case. 
Let $d^{\prime}$ be a metric given by Lemma 3.10. It has the tame growth of covering numbers. So for any $\varepsilon>0$ there exists an invariant probability measure $\mu$ on $\mathcal{X}$ satisfying

$$
\operatorname{mdim}(\mathcal{X}, \sigma) \leq \underline{\operatorname{rdim}}\left(\mathcal{X}, T, d^{\prime}, \mu\right)+\varepsilon
$$

Since $d^{\prime} \leq d$

$$
\underline{\operatorname{rdim}}\left(\mathcal{X}, T, d^{\prime}, \mu\right) \leq \underline{\operatorname{rdim}}(\mathcal{X}, T, d, \mu) .
$$

Since $\varepsilon>0$ is arbitrary, this proves the claim.

Corollary 3.14. If a dynamical system $(\mathcal{X}, T)$ has the marker property, then there exists a metric $d$ on $\mathcal{X}$ such that all the following quantities are equal to each other:

$$
\begin{aligned}
& \operatorname{mdim}(\mathcal{X}, T), \quad \operatorname{mdim}_{\mathrm{H}}(\mathcal{X}, T, d), \\
& \operatorname{mim}_{\mathrm{M}}(\mathcal{X}, T, d), \quad \underline{\operatorname{mim}_{\mathrm{M}}}(\mathcal{X}, T, d), \\
& \sup _{\mu \in \mathscr{M}^{T}(\mathcal{X})} \overline{\operatorname{rdim}}(\mathcal{X}, T, d, \mu), \quad \sup _{\mu \in \mathscr{M}^{T}(\mathcal{X})} \underline{\operatorname{dim}}(\mathcal{X}, T, d, \mu) .
\end{aligned}
$$

Proof. All the quantities in (3.4) are bounded between mdim $(\mathcal{X}, T)$ and $\overline{\operatorname{mdim}}_{\mathrm{M}}(\mathcal{X}, T, d)$ by Proposition 3.2 and Corollary 3.13. Take a metric $d$ given in Theorem 3.12. Then $\operatorname{mdim}(\mathcal{X}, T)=\overline{\operatorname{mdim}}_{\mathrm{M}}(\mathcal{X}, T, d)$ and hence all the quantities in (3.4) coincide with each other.

Now we can prove the double variational principle (Theorem 1.1).

Proof of Theorem 1.1. Let $(\mathcal{X}, T)$ be a dynamical system having the marker property. From Corollary 3.13

$$
\begin{aligned}
\operatorname{mdim}(\mathcal{X}, T) & \leq \inf _{d \in \mathscr{D}(\mathcal{X})} \sup _{\mu \in \mathscr{M}^{T}(\mathcal{X})} \underline{\operatorname{rdim}}(\mathcal{X}, T, d, \mu) \\
& \leq \inf _{d \in \mathscr{D}(\mathcal{X})} \sup _{\mu \in \mathscr{M}^{T}(\mathcal{X})} \overline{\operatorname{rdim}}(\mathcal{X}, T, d, \mu) .
\end{aligned}
$$

On the other hand we already know (Corollary 3.14) that there exists $d \in \mathscr{D}(\mathcal{X})$ satisfying

$$
\operatorname{mdim}(\mathcal{X}, T)=\sup _{\mu \in \mathscr{M}^{T}(\mathcal{X})} \overline{\operatorname{rdim}}(\mathcal{X}, T, d, \mu)=\sup _{\mu \in \mathscr{M}^{T}(\mathcal{X})} \underline{\operatorname{rdim}}(\mathcal{X}, T, d, \mu) .
$$

Remark 3.15. It follows from Proposition 3.2 and Theorem 3.11 that for a metric $d$ having the tame growth of covering numbers

$$
\begin{aligned}
& \operatorname{mdim}_{\mathrm{H}}(\mathcal{X}, T, d) \leq \sup _{\mu \in \mathscr{M}^{T}(\mathcal{X})} \overline{\operatorname{rdim}}(\mathcal{X}, T, d, \mu) \leq \overline{\operatorname{mdim}}_{\mathrm{M}}(\mathcal{X}, T, d), \\
& \operatorname{mdim}_{\mathrm{H}}(\mathcal{X}, T, d) \leq \sup _{\mu \in \mathscr{M}^{T}(\mathcal{X})} \underline{\operatorname{rdim}}(\mathcal{X}, T, d, \mu) \leq \underline{\operatorname{mim}}_{\mathrm{M}}(\mathcal{X}, T, d) .
\end{aligned}
$$


Hence we have a sufficient criterion (under the assumption of the tame growth of covering numbers) for the equality (1.8) in Problem 1.3. If mean Hausdorff dimension is equal to metric mean dimension, then they also coincide with the supremum of rate distortion dimensions.

\section{Proof of Theorem 3.11: Geometric measure theory And MISIUREWICZ'S TECHNIQUE}

We prove Theorem 3.11 in this section. The proof is a combination of geometric measure theory and the rate distortion theory version of Misiurewicz's technique [Mis76, LT18].

4.1. Geometric measure theory around Frostman's lemma. The purpose of this subsection is to prepare some basics of geometric measure theory around Frostman's lemma. Frostman's lemma is a fundamental result in geometric measure theory. It states that a Borel subset $A \subset \mathbb{R}^{n}$ has positive (possibly infinite) $s$-dimensional Hausdorff measure if and only if there exists a nonzero Radon measure $\mu$ on $\mathbb{R}^{n}$ supported on $A$ and satisfying $\mu\left(B_{r}(x)\right) \leq r^{s}$ for all $x \in \mathbb{R}^{n}$ and $r>0$ (see [Mat95, 8.8 Theorem]). We need a generalization of this result to compact metric spaces, which is due to Howroyd [H95]. Our presentation follows the book of Mattila [Mat95, Sections 8.14-8.17].

Let $(\mathcal{X}, d)$ be a compact metric space. For $\delta>0$ and $s \geq 0$ we define $\lambda_{\delta}^{s}(\mathcal{X}, d)$ as

$$
\inf \sum_{n=1}^{\infty} c_{n}\left(\operatorname{diam} E_{n}\right)^{s}
$$

where the infimum is taken over all countable families $\left\{\left(E_{n}, c_{n}\right)\right\}$ such that $0<c_{n}<\infty, E_{n} \subset \mathcal{X}$ with $\operatorname{diam} E_{n}<\delta$ and

$$
\forall x \in \mathcal{X}: \sum_{n=1}^{\infty} c_{n} 1_{E_{n}}(x) \geq 1 .
$$

Obviously $\lambda_{\delta}^{s}(\mathcal{X}, d) \leq \mathcal{H}_{\delta}^{s}(\mathcal{X}, d)$.

\section{Lemma 4.1.}

$$
\mathcal{H}_{6 \delta}^{s}(\mathcal{X}, d) \leq 6^{s} \lambda_{\delta}^{s}(\mathcal{X}, d)
$$

Proof. The proof is essentially the same as [Mat95, 8.16 Lemma], but the above statement is a bit different 13 (at least formally) from [Mat95, 8.16 Lemma]. So we include a proof.

\footnotetext{
${ }^{13} \mathrm{An}$ important point for us is that the statement is valid for each fixed $\delta$ (not only the limits of $\delta \rightarrow 0$ ).
} 
Claim 4.2. Let $a_{1}, \ldots, a_{N}$ and $m$ be positive integers. Let $B_{n}^{\circ}=$ $B_{r_{n}}^{\circ}\left(x_{n}\right)(1 \leq n \leq N)$ be open balls in $\mathcal{X}$ of radius $r_{n}<\delta$. If

$$
\mathcal{X}=\left\{\sum_{n=1}^{N} a_{n} 1_{B_{n}^{\circ}} \geq m\right\}
$$

then

$$
\mathcal{H}_{6 \delta}^{s}(\mathcal{X}, d) \leq m^{-1} 6^{s} \sum_{n=1}^{N} a_{n} r_{n}^{s}
$$

Proof. The induction on $m$ : If $m=1$ then $\mathcal{X}=\bigcup_{n=1}^{N} B_{n}^{\circ}$ and $\operatorname{diam} B_{n}^{\circ}<$ $2 \delta$. Hence

$$
\mathcal{H}_{2 \delta}^{s}(\mathcal{X}, d) \leq \sum_{n=1}^{N}\left(\operatorname{diam} B_{n}^{\circ}\right)^{s} \leq 2^{s} \sum_{n=1}^{N} r_{n}^{s} .
$$

Suppose $m \geq 2$. By Finite Vitali's covering lemma (see EW11, Lemma 2.27]) there exists a disjoint family $\mathcal{B} \subset\left\{B_{1}^{\circ}, \ldots, B_{N}^{\circ}\right\}$ satisfying $\mathcal{X}=$ $\bigcup_{B_{n}^{\circ} \in \mathcal{B}} 3 B_{n}^{\circ}$ where $3 B_{n}^{\circ}:=B_{3 r_{n}}^{\circ}\left(x_{n}\right)$. We have $\operatorname{diam}\left(3 B_{n}^{\circ}\right) \leq 6 r_{n}<6 \delta$ and hence

$$
\mathcal{H}_{6 \delta}^{s}(\mathcal{X}, d) \leq 6^{s} \sum_{B_{n}^{\circ} \in \mathcal{B}} r_{n}^{s}
$$

We set

$$
a_{n}^{\prime}= \begin{cases}a_{n} & \text { if } B_{n}^{\circ} \notin \mathcal{B} \\ a_{n}-1 & \text { if } B_{n}^{\circ} \in \mathcal{B} .\end{cases}
$$

Since $\mathcal{B}$ is a disjoint family, we have $\sum a_{n}^{\prime} 1_{B_{n}^{\circ}}(x) \geq m-1$ for all $x \in \mathcal{X}$. By the induction hypothesis,

$$
(m-1) \mathcal{H}_{6 \delta}^{s}(\mathcal{X}, d) \leq 6^{s} \sum_{n=1}^{N} a_{n}^{\prime} r_{n}^{s} .
$$

Thus

$$
m \mathcal{H}_{6 \delta}^{s}(\mathcal{X}, d) \leq 6^{s} \sum_{n=1}^{N} a_{n}^{\prime} r_{n}^{s}+6^{s} \sum_{B_{n}^{\circ} \in \mathcal{B}} r_{n}^{s}=6^{s} \sum_{n=1}^{N} a_{n} r_{n}^{s}
$$

Let $0<c_{n}<\infty, E_{n} \subset \mathcal{X}$ such that $\operatorname{diam} E_{n}<\delta$ and $\sum_{n=1}^{\infty} c_{n} 1_{E_{n}}(x) \geq$ 1 for all $x \in \mathcal{X}$. Let $\varepsilon>0$ and $0<t<1$. We choose diam $E_{n}<r_{n}<\delta$ satisfying

$$
\sum_{n=1}^{\infty} c_{n} r_{n}^{s}<\varepsilon+\sum_{n=1}^{\infty} c_{n}\left(\operatorname{diam} E_{n}\right)^{s}
$$


We pick $x_{n} \in E_{n}$. The open balls $B_{n}^{\circ}:=B_{r_{n}}^{\circ}\left(x_{n}\right)$ contain $E_{n}$ and hence

$$
\mathcal{X}=\bigcup_{N=1}^{\infty}\left\{\sum_{n=1}^{N} c_{n} 1_{B_{n}^{\circ}}>t\right\} \text {. }
$$

Each set $\{\cdots\}$ here is open. Since $\mathcal{X}$ is compact, we can find $N$ such that $\mathcal{X}=\left\{\sum_{n=1}^{N} c_{n} 1_{B_{n}^{\circ}}>t\right\}$. We choose rational numbers $0<$ $b_{n} \leq c_{n}$ so that $\mathcal{X}=\left\{\sum_{n=1}^{N} b_{n} 1_{B_{n}^{\circ}}>t\right\}$. Take a positive integer $p$ such that all $a_{n}:=p b_{n}$ become integers. Set $m=\lceil p t\rceil$. Then $\mathcal{X}=\left\{\sum_{n=1}^{N} a_{n} 1_{B_{n}^{\circ}} \geq m\right\}$. By Claim 4.2

$$
\mathcal{H}_{6 \delta}^{s}(\mathcal{X}, d) \leq m^{-1} 6^{s} \sum_{n=1}^{N} a_{n} r_{n}^{s} \leq p m^{-1} 6^{s} \sum_{n=1}^{N} b_{n} r_{n}^{s} \leq p m^{-1} 6^{s} \sum_{n=1}^{N} c_{n} r_{n}^{s} .
$$

It follows from $m \geq p t$ and (4.1) that

$$
\mathcal{H}_{6 \delta}^{s}(\mathcal{X}, d) \leq t^{-1} 6^{s}\left(\varepsilon+\sum_{n=1}^{\infty} c_{n}\left(\operatorname{diam} E_{n}\right)^{s}\right) .
$$

Let $\varepsilon \rightarrow 0$ and $t \rightarrow 1$. This proves the statement.

Lemma 4.3. There exists a Borel measure $\mu$ on $\mathcal{X}$ satisfying $\mu(X)=$ $\lambda_{\delta}^{s}(\mathcal{X}, d)$ and

$$
\mu(E) \leq(\operatorname{diam} E)^{s} \quad \text { for all } E \subset \mathcal{X} \text { with } \operatorname{diam} E<\delta .
$$

Sketch of the proof. See [Mat95, 8.17 Theorem] for the details. We define a sublinear functional $p(f)$ for continuous functions $f: \mathcal{X} \rightarrow \mathbb{R}$ by

$$
p(f)=\inf \sum_{n=1}^{\infty} c_{n}\left(\operatorname{diam}\left(E_{n}\right)\right)^{s}
$$

where the infimum is taken over all countable families $\left\{\left(E_{n}, c_{n}\right)\right\}$ such that $0<c_{n}<\infty, E_{n} \subset \mathcal{X}$ with $\operatorname{diam} E_{n}<\delta$ and

$$
\forall x \in \mathcal{X}: \sum_{n=1}^{\infty} c_{n} 1_{E_{n}}(x) \geq f(x) .
$$

We have $p(1)=\lambda_{\delta}(\mathcal{X}, d)$. By using the Hahn-Banach theorem, we can find a linear functional $L$ defined on the space of continuous functions in $\mathcal{X}$ such that $L(1)=p(1)$ and for any continuous function $f$ on $\mathcal{X}$

$$
-p(-f) \leq L(f) \leq p(f) \text {. }
$$

If $f \geq 0$ then $L(f) \geq-p(-f)=0$. So $L$ is a positive functional. It follows from the Riesz representation theorem that there exists a Borel 
measure $\mu$ on $\mathcal{X}$ satisfying $L(f)=\int_{\mathcal{X}} f d \mu$. We can easily check that $\mu$ satisfies the statement.

Corollary 4.4. Let $0<c<1$. We can choose $\delta_{0}=\delta_{0}(c) \in(0,1)$ independent of $(\mathcal{X}, d)$ so that for any $0<\delta \leq \delta_{0}$ there exists a Borel probability measure $\mu$ on $\mathcal{X}$ satisfying

$$
\mu(E) \leq(\operatorname{diam} E)^{c \operatorname{dim}_{\mathrm{H}}(\mathcal{X}, d, \delta)} \quad \text { for all } E \subset \mathcal{X} \text { with } \operatorname{diam} E<\delta / 6 .
$$

Proof. Take $0<\delta_{0}<1$ satisfying

$$
\left(\frac{1}{\delta_{0}}\right)^{\frac{1-c}{2 c}} \geq 6
$$

Let $0<\delta \leq \delta_{0}$. If $\operatorname{dim}_{\mathrm{H}}(\mathcal{X}, d, \delta)=0$ then the statement is trivial (the delta measure satisfies the claim; recall that we promised $0^{0}=1$ ). So we assume $\operatorname{dim}_{\mathrm{H}}(\mathcal{X}, d, \delta)>0$. From Lemma 4.3 it is enough to prove $\lambda_{\delta / 6}^{s}(\mathcal{X}, d) \geq 1$ for $s:=c \operatorname{dim}_{\mathrm{H}}(\mathcal{X}, d, \delta)$.

Set $t=\frac{1+c}{2} \operatorname{dim}_{\mathrm{H}}(\mathcal{X}, d, \delta)$. We have $t-s=\frac{1-c}{2 c} s$ and hence

$$
\begin{aligned}
\mathcal{H}_{\delta}^{s}(\mathcal{X}, d) & \geq\left(\frac{1}{\delta}\right)^{t-s} \mathcal{H}_{\delta}^{t}(\mathcal{X}, d) \\
& \geq\left(\frac{1}{\delta}\right)^{t-s} \quad\left(\text { by } t<\operatorname{dim}_{\mathrm{H}}(\mathcal{X}, d, \delta)\right) \\
& =\left(\frac{1}{\delta}\right)^{\frac{1-c}{2 c} s} \geq 6^{s} .
\end{aligned}
$$

Then $\lambda_{\delta / 6}^{s}(\mathcal{X}, d) \geq 1$ by Lemma 4.1 .

4.2. $L^{1}$-mean Hausdorff dimension and the condition of tame growth of covering numbers. We need a modification of mean Hausdorff dimension. Let $(\mathcal{X}, T)$ be a dynamical system with a metric $d$. For $N \geq 1$ we define a new metric $\bar{d}_{N}$ on $\mathcal{X}$ by

$$
\bar{d}_{N}(x, y)=\frac{1}{N} \sum_{n=0}^{N-1} d\left(T^{n} x, T^{n} y\right) .
$$

This metric is more closely connected to the distortion condition

$$
\mathbb{E}\left(\frac{1}{N} \sum_{n=0}^{N-1} d\left(T^{n} X, Y_{n}\right)\right)<\varepsilon
$$

in the definition of rate distortion function (see \$2.3) than $d_{N}(x, y)=$ $\max _{0 \leq n<N} d\left(T^{n} x, T^{n} y\right)$. The next lemma is a manifestation of this connection. (This will be used only in $\$ 6$, But it is conceptually a toy model of the proof of Theorem 3.11, ) 
Lemma 4.5. Let $\mu$ be a $T$-invariant probability measure on $\mathcal{X}$. Suppose that there exist $s \geq 0$ and $\delta>0$ such that for any $N \geq 1$

$$
\mu(E) \leq\left(\operatorname{diam}\left(E, \bar{d}_{N}\right)\right)^{s N} \quad \text { for all } E \subset \mathcal{X} \text { with } \operatorname{diam}\left(E, \bar{d}_{N}\right)<\delta \text {. }
$$

Then $\underline{\operatorname{rdim}}(\mathcal{X}, T, d, \mu) \geq s$.

Proof. Define $\Delta: \mathcal{X} \rightarrow \mathcal{X}^{N}$ by $\Delta(x)=\left(x, T x, \ldots, T^{N-1} x\right)$. We define a metric $\bar{d}_{N}$ on $\mathcal{X}^{N}$ by

$$
\bar{d}_{N}\left(\left(x_{0}, \ldots, x_{N-1}\right),\left(y_{0}, \ldots, y_{N-1}\right)\right)=\frac{1}{N} \sum_{n=0}^{N-1} d\left(x_{n}, y_{n}\right) .
$$

The push-forward measure $\Delta_{*} \mu$ on $\mathcal{X}^{N}$ satisfies

$$
\Delta_{*} \mu(E) \leq\left(\operatorname{diam}\left(E, \bar{d}_{N}\right)\right)^{s N} \quad \text { for all } E \subset \mathcal{X}^{N} \text { with } \operatorname{diam}\left(E, \bar{d}_{N}\right)<\delta \text {. }
$$

Let $\varepsilon>0$ with $2 \varepsilon \log (1 / \varepsilon) \leq \delta$. Let $X$ and $Y=\left(Y_{0}, \ldots, Y_{N-1}\right)$ be random variables such that all $X$ and $Y_{n}$ take values in $\mathcal{X}$ and satisfy

$$
\operatorname{Law} X=\mu, \quad \mathbb{E}\left(\frac{1}{N} \sum_{n=0}^{N-1} d\left(T^{n} X, Y_{n}\right)\right)<\varepsilon .
$$

This condition is equivalent to Law $\Delta(X)=\Delta_{*} \mu$ and $\mathbb{E} \bar{d}_{N}(\Delta(X), Y)<$ $\varepsilon$. So we apply Lemma 2.10 to $(\Delta(X), Y)$ and get

$$
\frac{I(X ; Y)}{N}=\frac{I(\Delta(X) ; Y)}{N} \geq s \log (1 / \varepsilon)-C(s+1),
$$

where $C>0$ is a universal constant. Therefore

$$
\begin{gathered}
R(d, \mu, \varepsilon) \geq s \log (1 / \varepsilon)-C(s+1), \\
\underline{\operatorname{rdim}}(\mathcal{X}, T, d, \mu)=\liminf _{\varepsilon \rightarrow 0} \frac{R(d, \mu, \varepsilon)}{\log (1 / \varepsilon)} \geq s .
\end{gathered}
$$

For a dynamical system $(\mathcal{X}, T)$ with a metric $d$, we define the $L^{1}$ mean Hausdorff dimension by

$$
\operatorname{mdim}_{\mathrm{H}, L^{1}}(\mathcal{X}, T, d)=\lim _{\varepsilon \rightarrow 0}\left(\limsup _{N \rightarrow \infty} \frac{\operatorname{dim}_{\mathrm{H}}\left(\mathcal{X}, \bar{d}_{N}, \varepsilon\right)}{N}\right) .
$$

Since $\bar{d}_{N} \leq d_{N}$, this always satisfies

$$
\operatorname{mdim}_{\mathrm{H}, L^{1}}(\mathcal{X}, T, d) \leq \operatorname{mdim}_{\mathrm{H}}(\mathcal{X}, T, d) .
$$

These two quantities actually coincide under the tame growth of covering numbers condition (this is the only place where we use the tame growth of covering numbers in the proof of Theorem 3.11): 
Lemma 4.6. If $(\mathcal{X}, d)$ has the tame growth of covering numbers, then $\operatorname{mdim}_{\mathrm{H}, L^{1}}(\mathcal{X}, T, d)=\operatorname{mdim}_{\mathrm{H}}(\mathcal{X}, T, d)$.

Proof. We assume $\operatorname{mdim}_{\mathrm{H}, L^{1}}(\mathcal{X}, T, d)<\infty$ and prove $\operatorname{mdim}_{\mathrm{H}}(\mathcal{X}, T, d) \leq$ $\operatorname{mdim}_{\mathrm{H}, L^{1}}(\mathcal{X}, T, d)$. We set $[N]=\{0,1,2, \ldots, N-1\}$ and define a metric $d_{A}$ on $\mathcal{X}$ for $A \subset[N]$ by $d_{A}(x, y)=\max _{a \in A} d\left(T^{a} x, T^{a} y\right)$. In particular $d_{N}=d_{[N]}$.

For $\tau>0$ we set $M(\tau)=\#(\mathcal{X}, d, \tau)$. We can find a covering $\mathcal{X}=$ $W_{1}^{\tau} \cup \cdots \cup W_{M(\tau)}^{\tau}$ with $\operatorname{diam}\left(W_{m}^{\tau}, d\right)<\tau$ for all $1 \leq m \leq M(\tau)$. Take any $0<\delta<1 / 2$ and $s>\operatorname{mdim}_{\mathrm{H}, L^{1}}(\mathcal{X}, T, d)$. By the tame growth of covering numbers, we can choose $0<\varepsilon_{0}<1$ satisfying

- $\tau^{\delta} \log M(\tau)<1$ for $0<\tau<\varepsilon_{0}$.

- $4 \cdot 2^{s /(1-2 \delta)} \cdot \varepsilon_{0}^{\delta s /(1-2 \delta)}<1$.

Let $0<\varepsilon<\varepsilon_{0}$. Let $N$ be a sufficiently large number. From $\operatorname{mdim}_{\mathrm{H}, L^{1}}(\mathcal{X}, T, d)<s$ we can find a covering $\mathcal{X}=\bigcup_{n=1}^{\infty} E_{n}$ satisfy$\operatorname{ing} \tau_{n}:=\operatorname{diam}\left(E_{n}, \bar{d}_{N}\right)<\varepsilon$ for all $n$ and

$$
\sum_{n=1}^{\infty} \tau_{n}^{s N}<1 .
$$

Set $L_{n}=\left(1 / \tau_{n}\right)^{\delta}$. Pick a point $x_{n}$ from each $E_{n}$. Every point $x \in E_{n}$ satisfies $\bar{d}_{N}\left(x, x_{n}\right) \leq \tau_{n}$ and hence

$$
\left|\left\{k \in[N] \mid d\left(T^{k} x, T^{k} x_{n}\right) \geq L_{n} \tau_{n}\right\}\right| \leq \frac{N}{L_{n}} .
$$

Thus there exists $A \subset[N]$ (depending on $x \in E_{n}$ ) satisfying $|A| \leq$ $N / L_{n}$ and $d_{[N] \backslash A}\left(x, x_{n}\right)<L_{n} \tau_{n}$. This implies

$$
E_{n} \subset \bigcup_{A \subset[N],|A| \leq N / L_{n}} B_{L_{n} \tau_{n}}^{\circ}\left(x_{n}, d_{[N] \backslash A}\right) .
$$

Here $B_{L_{n} \tau_{n}}^{\circ}\left(x_{n}, d_{[N] \backslash A}\right)$ is the open ball of radius $L_{n} \tau_{n}$ with respect to $d_{[N] \backslash A}$ around $x_{n}$, which for $A=\left\{a_{1}, \ldots, a_{r}\right\}$ we can write as

$$
\bigcup_{1 \leq i_{1}, \ldots, i_{r} \leq M\left(\tau_{n}\right)} B_{L_{n} \tau_{n}}^{\circ}\left(x_{n}, d_{[N] \backslash A}\right) \cap T^{-a_{1}} W_{i_{1}}^{\tau_{n}} \cap \cdots \cap T^{-a_{r}} W_{i_{r}}^{\tau_{n}} .
$$

Therefore $\mathcal{X}$ can be written as a union of

$$
B_{L_{n} \tau_{n}}^{\circ}\left(x_{n}, d_{[N] \backslash A}\right) \cap T^{-a_{1}} W_{i_{1}}^{\tau_{n}} \cap \cdots \cap T^{-a_{r}} W_{i_{r}}^{\tau_{n}},
$$

where $n \geq 1, A=\left\{a_{1}, \ldots, a_{r}\right\} \subset[N]$ with $r \leq N / L_{n}$ and $1 \leq$ $i_{1}, \ldots, i_{r} \leq M\left(\tau_{n}\right)$. The diameter of (4.2) with respect to $d_{N}$ is bounded 
by $2 L_{n} \tau_{n}=2 \tau_{n}^{1-\delta}<2 \varepsilon^{1-\delta}$. Thus

$$
\mathcal{H}_{2 \varepsilon^{1-\delta}}^{s N /(1-2 \delta)}\left(\mathcal{X}, d_{N}\right) \leq \sum_{n=1}^{\infty} 2^{N} M\left(\tau_{n}\right)^{N / L_{n}}\left(2 \tau_{n}^{1-\delta}\right)^{s N /(1-2 \delta)} .
$$

Here $2^{N}$ comes from the choice of $A \subset[N]$.

$$
2^{N} M\left(\tau_{n}\right)^{N / L_{n}}\left(2 \tau_{n}^{1-\delta}\right)^{s N /(1-2 \delta)}=\left\{2^{1+\frac{s}{1-2 \delta}} M\left(\tau_{n}\right)^{\tau_{n}^{\delta}} \tau_{n}^{\frac{s \delta}{1-2 \delta}}\right\}^{N} \tau_{n}^{s N} .
$$

Recall $\tau_{n}<\varepsilon<\varepsilon_{0}$ and the choice of $\varepsilon_{0}$ above. We have

$$
2^{1+\frac{s}{1-2 \delta}} M\left(\tau_{n}\right)^{\tau_{n}^{\delta}} \tau_{n}^{\frac{s \delta}{1-2 \delta}}<4 \cdot 2^{\frac{s}{1-2 \delta}} \varepsilon_{0}^{\frac{s \delta}{1-2 \delta}}<1 .
$$

Therefore

$$
\mathcal{H}_{2 \varepsilon^{1-\delta}}^{s N /(1-2 \delta)}\left(\mathcal{X}, d_{N}\right)<\sum_{n=1}^{\infty} \tau_{n}^{s N}<1
$$

So we get

$$
\operatorname{dim}_{\mathrm{H}}\left(\mathcal{X}, d_{N}, 2 \varepsilon^{1-\delta}\right) \leq \frac{s N}{1-2 \delta} .
$$

Since this holds for any sufficiently large $N$,

$$
\limsup _{N \rightarrow \infty}\left(\frac{1}{N} \operatorname{dim}_{\mathrm{H}}\left(\mathcal{X}, d_{N}, 2 \varepsilon^{1-\delta}\right)\right) \leq \frac{s}{1-2 \delta} .
$$

Here $0<\varepsilon<\varepsilon_{0}$ is arbitrary. Thus

$$
\operatorname{mim}_{\mathrm{H}}(\mathcal{X}, T, d) \leq \frac{s}{1-2 \delta}
$$

Letting $\delta \rightarrow 0$ and $s \rightarrow \operatorname{mdim}_{\mathrm{H}, L^{1}}(\mathcal{X}, T, d)$, we get the statement.

Remark 4.7. The same argument also proves that the lower mean Hausdorff dimension $\underline{\operatorname{mdim}}_{\mathrm{H}}(\mathcal{X}, T, d)$ (see Remark 3.1) coincides with

$$
\lim _{\varepsilon \rightarrow 0}\left(\liminf _{N \rightarrow \infty} \frac{\operatorname{dim}_{\mathrm{H}}\left(\mathcal{X}, \bar{d}_{N}, \varepsilon\right)}{N}\right)
$$

if $(\mathcal{X}, d)$ has the tame growth of covering numbers.

Remark 4.8. In [LT18, Lemmas 25 and 26] we showed a similar result for metric mean dimension. In [LT18, Lemma 25] we proved the following statement: Let $(\mathcal{X}, T)$ be a dynamical system with a metric $d$. For any integer $N \geq 1$ and real numbers $\varepsilon>0$ and $L>1$, we have

$$
\log \#\left(\mathcal{X}, \bar{d}_{N}, \varepsilon\right) \geq \log \#\left(\mathcal{X}, d_{N}, 2 L \varepsilon\right)-N-\frac{N}{L} \log \#(\mathcal{X}, d, \varepsilon) .
$$

This will be used in $\$ 6$. 
4.3. Proof of Theorem 3.11. Theorem 3.11 follows from Lemma 4.6 and the next theorem.

Theorem 4.9. For any dynamical system $(\mathcal{X}, T)$ with a metric $d$

$$
\operatorname{mdim}_{\mathrm{H}, L^{1}}(\mathcal{X}, T, d) \leq \sup _{\mu \in \mathscr{M}^{T}(\mathcal{X})} \underline{\operatorname{rdim}}(\mathcal{X}, T, d, \mu) .
$$

Notice that here we do not assume the condition of the tame growth of covering numbers.

We use the following elementary lemma.

Lemma 4.10. Let $A$ be a finite set and $\left\{\mu_{n}\right\}$ a sequence of probability measures on $A$. Suppose $\mu_{n}$ converges to some $\mu$ in the weak* topology ${ }^{14}$. Then there exists a sequence of probability measures $\pi_{n}$ on $A \times A$ such that

- $\pi_{n}$ is a coupling between $\mu_{n}$ and $\mu$, namely its first and second marginals are equal to $\mu_{n}$ and $\mu$ respectively.

- $\pi_{n}$ converges to $(\mathrm{id} \times \mathrm{id})_{*} \mu$ in the weak $k^{*}$ topology, namely

$$
\pi_{n}(a, b) \rightarrow\left\{\begin{array}{ll}
\mu(a) & (\text { if } a=b) \\
0 & (\text { if } a \neq b)
\end{array} .\right.
$$

Proof. This follows from a much more general fact on optimal transport that the Wasserstein distance metrizes the weak* topology Vil09, Theorem 6.9]. See [LT18, Appendix] for an elementary self-contained proof.

As in the proof of Lemma 4.5, we extend the definition of $\bar{d}_{n}$. For $x=\left(x_{0}, \ldots, x_{n-1}\right)$ and $y=\left(y_{0}, \ldots, y_{n-1}\right)$ in $\mathcal{X}^{n}$ we set

$$
\bar{d}_{n}(x, y)=\frac{1}{n} \sum_{k=0}^{n-1} d\left(x_{k}, y_{k}\right) .
$$

Proof of Theorem 4.9. We can assume $\operatorname{mdim}_{\mathrm{H}, L^{1}}(\mathcal{X}, T, d)>0$. Let $0<$ $s<\operatorname{mdim}_{\mathrm{H}, L^{1}}(\mathcal{X}, T, d)$. We will prove that there exists $\mu \in \mathscr{M}^{T}(\mathcal{X})$ satisfying $\underline{\operatorname{rdim}}(\mathcal{X}, T, d, \mu) \geq s$.

Take $0<c<1$ with $c \cdot \operatorname{mdim}_{\mathrm{H}, L^{1}}(\mathcal{X}, T, d)>s$ and let $\delta_{0}=\delta_{0}(c) \in$ $(0,1)$ be the constant given by Corollary 4.4. There exist $0<\delta<\delta_{0}$ and $n_{1}<n_{2}<n_{3}<\ldots$ satisfying $c \cdot \operatorname{dim}_{\mathrm{H}}\left(\mathcal{X}, \bar{d}_{n_{k}}, \delta\right)>s n_{k}$. By Corollary 4.4 we can find Borel probability measures $\nu_{k}$ on $\mathcal{X}$ satisfying

$$
\nu_{k}(E) \leq\left(\operatorname{diam}\left(E, \bar{d}_{n_{k}}\right)\right)^{s n_{k}}, \quad \forall E \subset \mathcal{X} \text { with } \operatorname{diam}\left(E, \bar{d}_{n_{k}}\right)<\frac{\delta}{6}
$$

\footnotetext{
${ }^{14}$ Since we assume that $A$ is a finite set, this just means that $\mu_{n}(x) \rightarrow \mu(x)$ at each $x \in A$
} 
Set

$$
\mu_{k}=\frac{1}{n_{k}} \sum_{n=0}^{n_{k}-1} T_{*}^{n} \nu_{k}
$$

By choosing a subsequence (also denoted by $\mu_{k}$ ), we can assume that $\mu_{k}$ converges to some $T$-invariant probability measure $\mu$ on $\mathcal{X}$ in the weak* topology. We will show $\underline{\operatorname{rdim}}(\mathcal{X}, T, d, \mu) \geq s$.

Let $\varepsilon$ be any positive number with $2 \varepsilon \log (1 / \varepsilon) \leq \delta / 10$. We would like to prove an estimate such as

$$
R(d, \mu, \varepsilon) \geq s \log (1 / \varepsilon)+\text { small error terms. }
$$

For this purpose, let $X$ and $Y=\left(Y_{0}, \ldots, Y_{m-1}\right)$ be coupled random variables such that $X, Y_{0}, \ldots, Y_{m-1}$ take values in $\mathcal{X}$ and satisfy

$$
\operatorname{Law} X=\mu, \quad \mathbb{E}\left(\frac{1}{m} \sum_{j=0}^{m-1} d\left(T^{j} X, Y_{j}\right)\right)<\varepsilon .
$$

We need to show $I(X ; Y) \geq s m \log (1 / \varepsilon)+$ small error terms. As we remarked in Remark 2.11, we can assume that $Y$ takes only finitely many values. Let $\mathcal{Y} \subset \mathcal{X}^{m}$ be the set of possible values of $Y$.

Idea of the proof. Here we roughly explain the idea of the proof, assuming $m=1$. That is to say, we give a lower bound on $I(X ; Y)$ if Law $X=\mu$ and $Y$ is any $\mathcal{X}$-valued random variable coupled to it with $\mathbb{E} d(X, Y)<\varepsilon$.

Since $\mu_{k} \rightarrow \mu$, we can find random variables $X(k)$ coupled to $X$ such that $X(k)$ take values in $\mathcal{X}$ with $\operatorname{Law} X(k)=\mu_{k}$ and

$$
\mathbb{E} d(X, X(k)) \rightarrow 0, \quad I(X(k) ; Y) \rightarrow I(X ; Y) .
$$

(Here we have ignored a technical problem. The above convergence $I(X(k) ; Y) \rightarrow I(X ; Y)$ essentially follows from Lemma 2.6. But Lemma 2.6 is valid only if the underlying space is a finite set. We will address this issue by introducing an appropriate finite partition $\mathcal{P}$ below. At the moment we pretend that $X$ and $X(k)$ take values in a finite set.)

Using this coupling between $X(k)$ and $Y$, we will construct a new coupling between $Z(k)=\left(X^{\prime}(k), T X^{\prime}(k), \ldots, T^{n_{k}-1} X^{\prime}(k)\right)$ with $X^{\prime}(k)$ a random variable taking values in $\mathcal{X}$ and obeying $\nu_{k}$, and a new random variable $W(k)=\left(W(k)_{0}, \ldots, W(k)_{n_{k}-1}\right)$ so that

$$
\mathbb{E} \bar{d}_{n_{k}}(Z(k), W(k))<\varepsilon
$$

and $I(Z(k) ; W(k))$ is bounded from above using $I(X ; Y)$. As we can bound $I(Z(k) ; W(k))$ from bellow using Lemma 2.10 this will give us the desired lower bound on $I(X ; Y)$. 
We define the random variable $W(k)=\left(W(k)_{0}, \ldots, W(k)_{n_{k}-1}\right)$, coupled to $Z(k)$ and taking values in $\mathcal{X}^{n_{k}}$, as follows. Let $\rho_{k}(y \mid x)=$ $\mathbb{P}(Y=y \mid X(k)=x)$. Then the law of $W(k)=\left(W(k)_{0}, \ldots, W(k)_{n_{k}-1}\right)$ is determined by requiring that $W(k)_{0}, \ldots, W(k)_{n_{k}-1}$ are conditionally independent given $Z(k)$ and

$$
\mathbb{P}\left(W(k)_{n}=y \mid T^{n} X^{\prime}(k)=x\right)=\rho_{k}(y \mid x) .
$$

By (4.4) we have that

$$
\mathbb{E} \bar{d}_{n_{k}}(Z(k), W(k))=\mathbb{E} d(X(k), Y) \rightarrow \mathbb{E} d(X, Y)<\varepsilon,
$$

so that indeed $\mathbb{E} \bar{d}_{n_{k}}(Z(k), W(k))<\varepsilon$ for large $k$.

Since $W(k)_{0}, \ldots, W(k)_{n_{k}-1}$ are conditionally independent given $Z(k)$, we use the subadditivity of mutual information (Lemma 2.7) and get

$$
I(Z(k) ; W(k)) \leq \sum_{n=0}^{n_{k}-1} I\left(Z(k) ; W(k)_{n}\right)=\sum_{n=0}^{n_{k}-1} I\left(T^{n} X^{\prime}(k) ; W(k)_{n}\right) .
$$

By definition of $W(k)_{n}$, we have $I\left(T^{n} X^{\prime}(k) ; W(k)_{n}\right)=I\left(T_{*}^{n} \nu_{k}, \rho_{k}\right)$ in the notation of Lemma 2.8, Recall $\mu_{k}=\left(1 / n_{k}\right) \sum_{n=0}^{n_{k}-1} T_{*}^{n} \nu_{k}$. By using the concavity property of mutual information given in Lemma 2.8 it follows that

$$
\frac{1}{n_{k}} \sum_{n=0}^{n_{k}-1} I\left(T_{*}^{n} \nu_{k}, \rho_{k}\right) \leq I\left(\mu_{k}, \rho_{k}\right)=I(X(k) ; Y) .
$$

Thus we get

$$
I(Z(k) ; W(k)) \leq n_{k} I(X(k) ; Y)
$$

Since Law $X^{\prime}(k)=\nu_{k}$, it follows from (4.3) and Lemma 2.10 that

$$
I(Z(k) ; W(k)) \geq s n_{k} \log (1 / \varepsilon)+\text { small error terms, }
$$

hence by (4.5) we see that

$$
I(X(k) ; Y) \geq s \log (1 / \varepsilon)+\text { small error terms. }
$$

As $I(X(k) ; Y) \rightarrow I(X ; Y)$ by (4.4)

$$
I(X ; Y) \geq s \log (1 / \varepsilon)+\text { small error terms. }
$$

This is what we want to prove.

Now we return to the proof. Recall our situation: The random variables $X$ and $Y=\left(Y_{0}, \ldots, Y_{m-1}\right)$ take values in $\mathcal{X}$ and a finite set $\mathcal{Y} \subset \mathcal{X}^{m}$ respectively. They satisfy Law $X=\mu$ and

$$
\frac{1}{m} \mathbb{E}\left(\sum_{j=0}^{m-1} d\left(T^{j} X, Y_{j}\right)\right)<\varepsilon
$$


We want to estimate $I(X ; Y)$ from below.

We choose a positive number $\tau$ satisfying

$$
\tau \leq \min \left(\frac{\varepsilon}{3}, \frac{\delta}{20}\right), \quad \frac{\tau}{2}+\mathbb{E}\left(\frac{1}{m} \sum_{j=0}^{m-1} d\left(T^{j} X, Y_{j}\right)\right)<\varepsilon
$$

We take a finite partition $\mathcal{P}=\left\{P_{1}, \ldots, P_{L}\right\}$ of $\mathcal{X}$ such that for all $1 \leq l \leq L$

$$
\operatorname{diam}\left(P_{l}, d\right)<\frac{\tau}{2}, \quad \mu\left(\partial P_{l}\right)=0
$$

Pick a point $p_{l}$ from each $P_{l}$ and set $A=\left\{p_{1}, \ldots, p_{L}\right\}$. We define a map $\mathcal{P}: \mathcal{X} \rightarrow A$ by $\mathcal{P}\left(P_{l}\right)=\left\{p_{l}\right\}$. For $n \geq 1$ we define a map $\mathcal{P}^{n}: \mathcal{X} \rightarrow A^{n}$ by $\mathcal{P}^{n}(x)=\left(\mathcal{P}(x), \mathcal{P}(T x), \ldots, \mathcal{P}\left(T^{n-1} x\right)\right)$.

Claim 4.11. $\mathcal{P}_{*}^{n_{k}} \nu_{k}(E) \leq\left(\tau+\operatorname{diam}\left(E, \bar{d}_{n_{k}}\right)\right)^{s n_{k}}$ for all $E \subset A^{n_{k}}$ with $\operatorname{diam}\left(E, \bar{d}_{n_{k}}\right)<\delta / 10$.

Proof. We have $\mathcal{P}_{*}^{n_{k}} \nu_{k}(E)=\nu_{k}\left(\left(\mathcal{P}^{n_{k}}\right)^{-1} E\right)$. Since $\tau \leq \delta / 20$

$$
\operatorname{diam}\left(\left(\mathcal{P}^{n_{k}}\right)^{-1} E, \bar{d}_{n_{k}}\right)<\tau+\operatorname{diam}\left(E, \bar{d}_{n_{k}}\right)<\frac{\delta}{6}
$$

Then by (4.3)

$$
\begin{aligned}
\nu_{k}\left(\left(\mathcal{P}^{n_{k}}\right)^{-1} E\right) & \leq\left(\operatorname{diam}\left(\left(\mathcal{P}^{n_{k}}\right)^{-1} E, \bar{d}_{n_{k}}\right)\right)^{s n_{k}} \\
& <\left(\tau+\operatorname{diam}\left(E, \bar{d}_{n_{k}}\right)\right)^{s n_{k}} .
\end{aligned}
$$

It follows from $\mu_{k} \rightarrow \mu$ and $\mu\left(\partial P_{l}\right)=0$ that $\mathcal{P}_{*}^{m} \mu_{k} \rightarrow \mathcal{P}_{*}^{m} \mu$ as $k \rightarrow \infty$. Then by Lemma 4.10 there exists a sequence of couplings $\pi_{k}$ between $\mathcal{P}_{*}^{m} \mu_{k}$ and $\mathcal{P}_{*}^{m} \mu$ converging to $(\mathrm{id} \times \mathrm{id})_{*} \mathcal{P}_{*}^{m} \mu$. We take a random variable $X(k)$ coupled to $\mathcal{P}^{m}(X)$ such that $X(k)$ takes values in $A^{m}$ with Law $\left(X(k), \mathcal{P}^{m}(X)\right)=\pi_{k}$. (In particular $X(k)$ obeys $\mathcal{P}_{*}^{m} \mu_{k}$.) This satisfies

$$
\mathbb{E} \bar{d}_{m}\left(X(k), \mathcal{P}^{m}(X)\right) \rightarrow 0, \quad I(X(k) ; Y) \rightarrow I\left(\mathcal{P}^{m}(X) ; Y\right)
$$


The latter condition 15 follows from Lemma 2.6. Since $\operatorname{diam}\left(\mathcal{P}_{l}, d\right)<\tau / 2$,

$$
\begin{aligned}
\mathbb{E} \bar{d}_{m}(X(k), Y) & <\mathbb{E} \bar{d}_{m}\left(X(k), \mathcal{P}^{m}(X)\right)+\frac{\tau}{2}+\mathbb{E}\left(\frac{1}{m} \sum_{j=0}^{m-1} d\left(T^{j} X, Y_{j}\right)\right) \\
& \rightarrow \frac{\tau}{2}+\mathbb{E}\left(\frac{1}{m} \sum_{j=0}^{m-1} d\left(T^{j} X, Y_{j}\right)\right)<\varepsilon \quad \text { by (4.6). }
\end{aligned}
$$

For $x=\left(x_{0}, \ldots, x_{n-1}\right) \in \mathcal{X}^{n}$ and $0 \leq a \leq b<n$ we denote $x_{a}^{b}=$ $\left(x_{a}, x_{a+1}, \ldots, x_{b}\right)$. For $x, y \in \mathcal{X}^{m}$ with $\mathcal{P}_{*}^{m} \mu_{k}(x)>0$ we consider a conditional probability mass function

$$
\rho_{k}(y \mid x)=\mathbb{P}(Y=y \mid X(k)=x) .
$$

Fix a point $a \in \mathcal{X}$. Let $n_{k}=m q+r$ with $m \leq r<2 m$. For $x, y \in \mathcal{X}^{n_{k}}$ and $0 \leq j<m$ we define a conditional probability mass function

$$
\sigma_{k, j}(y \mid x)=\prod_{i=0}^{q-1} \rho_{k}\left(y_{j+i m}^{j+i m+m-1} \mid x_{j+i m}^{j+i m+m-1}\right) \cdot \prod_{n \in[0, j) \cup\left[m q+j, n_{k}\right)} \delta_{a}\left(y_{n}\right) .
$$

Here $\delta_{a}(\cdot)$ is the delta probability measure at $a$ on $\mathcal{X}$. We set

$$
\sigma_{k}(y \mid x)=\frac{\sigma_{k, 0}(y \mid x)+\sigma_{k, 1}(y \mid x)+\cdots+\sigma_{k, m-1}(y \mid x)}{m} .
$$

This is defined for $x \in \mathcal{X}^{n_{k}}$ with $\mathcal{P}_{*}^{n_{k}} \nu_{k}(x)>0$.

Let $X^{\prime}(k)$ be a random variable taking values in $\mathcal{X}$ and obeying $\nu_{k}$. We set $Z(k)=\mathcal{P}^{n_{k}}\left(X^{\prime}(k)\right)$. We take a random variable $W(k)$ coupled to $Z(k)$ and taking values in $\mathcal{X}^{n_{k}}$ with

$$
\mathbb{P}(W(k)=y \mid Z(k)=x)=\sigma_{k}(y \mid x) .
$$

For $0 \leq j<m$ we also take a random variable $W(k, j)$ coupled to $Z(k)$ and taking values in $\mathcal{X}^{n_{k}}$ with

$$
\mathbb{P}(W(k, j)=y \mid Z(k)=x)=\sigma_{k, j}(y \mid x) .
$$

Claim 4.12. $\mathbb{E} \bar{d}_{n_{k}}(Z(k), W(k))<\varepsilon$ for large $k$.

Proof.

$$
\mathbb{E} \bar{d}_{n_{k}}(Z(k), W(k))=\frac{1}{m} \sum_{j=0}^{m-1} \mathbb{E} \bar{d}_{n_{k}}(Z(k), W(k, j)) .
$$

\footnotetext{
${ }^{15}$ The coupling between $X(k)$ and $Y$ is given by the probability mass function

$$
\sum_{x^{\prime} \in A^{m}} \pi_{k}\left(x, x^{\prime}\right) \mathbb{P}\left(Y=y \mid \mathcal{P}^{m}(X)=x^{\prime}\right),
$$
}

which converges to $\mathbb{P}\left(\mathcal{P}^{m}(X)=x, Y=y\right)$. 
$\bar{d}_{n_{k}}(Z(k), W(k, j))$ is bounded by

$$
\frac{r \cdot \operatorname{diam}(\mathcal{X}, d)}{n_{k}}+\frac{m}{n_{k}} \sum_{i=0}^{q-1} \bar{d}_{m}\left(\mathcal{P}^{m}\left(T^{j+i m} X^{\prime}(k)\right), W(k, j)_{j+i m}^{j+i m+m-1}\right) .
$$

$\mathbb{E} \bar{d}_{m}\left(\mathcal{P}^{m}\left(T^{j+i m} X^{\prime}(k)\right), W(k, j)_{j+i m}^{j+i m+m-1}\right)$ is equal to

$$
\sum_{x, y \in \mathcal{X}^{m}} \bar{d}_{m}(x, y) \rho_{k}(y \mid x) \mathcal{P}_{*}^{m} T_{*}^{j+i m} \nu_{k}(x) .
$$

Therefore $\mathbb{E} \bar{d}_{n_{k}}(Z(k), W(k))$ is bounded by

$$
\begin{aligned}
& \frac{r \cdot \operatorname{diam}(\mathcal{X}, d)}{n_{k}}+\sum_{x, y \in \mathcal{X}^{m}} \bar{d}_{m}(x, y) \rho_{k}(y \mid x)\left(\frac{1}{n_{k}} \sum_{\substack{0 \leq i<q \\
0 \leq j<m}} \mathcal{P}_{*}^{m} T_{*}^{j+i m} \nu_{k}(x)\right) \\
\leq & \frac{r \cdot \operatorname{diam}(\mathcal{X}, d)}{n_{k}}+\sum_{x, y \in \mathcal{X}^{m}} \bar{d}_{m}(x, y) \rho_{k}(y \mid x)\left(\frac{1}{n_{k}} \sum_{n=0}^{n_{k}-1} \mathcal{P}_{*}^{m} T_{*}^{n} \nu_{k}(x)\right) \\
= & \frac{r \cdot \operatorname{diam}(\mathcal{X}, d)}{n_{k}}+\sum_{x, y \in \mathcal{X}^{m}} \bar{d}_{m}(x, y) \rho_{k}(y \mid x) \mathcal{P}_{*}^{m} \mu_{k}(x) \\
= & \frac{r \cdot \operatorname{diam}(\mathcal{X}, d)}{n_{k}}+\mathbb{E} \bar{d}_{m}(X(k), Y) .
\end{aligned}
$$

From $r \leq 2 m$ and (4.8), this is less than $\varepsilon$ for large $k$.

\section{Claim 4.13.}

$$
\frac{1}{n_{k}} I(Z(k) ; W(k)) \leq \frac{1}{m} I(X(k) ; Y)
$$

Proof. By the convexity of mutual information (Lemma 2.8)

$$
I(Z(k) ; W(k)) \leq \frac{1}{m} \sum_{j=0}^{m-1} I(Z(k) ; W(k, j)) .
$$

By the subadditivity of mutual information under conditional independence (Lemma 2.7)

$$
I(Z(k) ; W(k, j)) \leq \sum_{i=0}^{q-1} I\left(Z(k) ; W(k, j)_{j+i m}^{j+i m+m-1}\right) .
$$

The term $I\left(Z(k) ; W(k, j)_{j+i m}^{j+i m+m-1}\right)$ is equal to

$$
I\left(\mathcal{P}^{m}\left(T^{j+i m} X^{\prime}(k)\right) ; W(k, j)_{j+i m}^{j+i m+m-1}\right)=I\left(\mathcal{P}_{*}^{m} T_{*}^{j+i m} \nu_{k}, \rho_{k}\right) .
$$


Hence

$$
\begin{aligned}
\frac{m}{n_{k}} I(Z(k) ; W(k)) \leq & \frac{1}{n_{k}} \sum_{\substack{0 \leq j<m \\
0 \leq i<q}} I\left(\mathcal{P}_{*}^{m}\left(T_{*}^{j+i m} \nu_{k}\right), \rho_{k}\right) \\
\leq & \frac{1}{n_{k}} \sum_{n=0}^{n_{k}-1} I\left(\mathcal{P}_{*}^{m} T_{*}^{n} \nu_{k}, \rho_{k}\right) \\
\leq & I\left(\frac{1}{n_{k}} \sum_{n=0}^{n_{k}-1} \mathcal{P}_{*}^{m} T_{*}^{n} \nu_{k}, \rho_{k}\right) \\
& \quad \text { by the concavity in Lemma 2.8 } \\
= & I\left(\mathcal{P}_{*}^{m} \mu_{k}, \rho_{k}\right) \\
= & I(X(k) ; Y) .
\end{aligned}
$$

Recall $2 \varepsilon \log (1 / \varepsilon) \leq \delta / 10, \tau \leq \min (\varepsilon / 3, \delta / 20)$ and $\operatorname{Law} Z(k)=$ $\mathcal{P}_{*}^{n_{k}} \nu_{k}$. We apply Lemma 2.10 with Claims 4.11 and 4.12 to $(Z(k), W(k))$ :

$$
I(Z(k) ; W(k)) \geq s n_{k} \log (1 / \varepsilon)-C\left(s n_{k}+1\right) \quad \text { for large } k .
$$

Here $C$ is a universal positive constant. From Claim 4.13

$$
\frac{1}{m} I(X(k) ; Y) \geq s \log (1 / \varepsilon)-C\left(s+\frac{1}{n_{k}}\right) .
$$

We know $I(X(k) ; Y) \rightarrow I\left(\mathcal{P}^{m}(X) ; Y\right)$ as $k \rightarrow \infty$ in (4.7). Hence

$$
\frac{1}{m} I\left(\mathcal{P}^{m}(X) ; Y\right) \geq s \log (1 / \varepsilon)-C s .
$$

By the data-processing inequality (Lemma 2.5)

$$
\frac{1}{m} I(X ; Y) \geq \frac{1}{m} I\left(\mathcal{P}^{m}(X) ; Y\right) \geq s \log (1 / \varepsilon)-C s .
$$

Therefore for any positive number $\varepsilon$ with $2 \varepsilon \log (1 / \varepsilon) \leq \delta / 10$

$$
R(d, \mu, \varepsilon) \geq s \log (1 / \varepsilon)-C s .
$$

Thus

$$
\underline{\operatorname{rdim}}(\mathcal{X}, T, d, \mu)=\liminf _{\varepsilon \rightarrow 0} \frac{R(d, \mu, \varepsilon)}{\log (1 / \varepsilon)} \geq s
$$

\section{Proof of Theorem 3.12}

The purpose of this section is to prove Theorem 3.12. The proof does not involve rate distortion theory, and in particular it is independent of $\$ 4$. 
5.1. Background: Pontrjagin-Schnirelmann's theorem. Theorems 2.3 and 3.12 look quite similar. Theorem 2.3 claims the existence of $d$ satisfying $\underline{\operatorname{mim}}_{\mathrm{M}}(\mathcal{X}, T, d)=\operatorname{mdim}(\mathcal{X}, T)$ wheres Theorem 3.12 claims the existence of $d$ satisfying $\overline{\operatorname{mdim}}_{\mathrm{M}}(\mathcal{X}, T, d)=\operatorname{mdim}(\mathcal{X}, T)$. But indeed the upper case (Theorem 3.12 ) is substantially subtler. The difficulty is already visible in a classical, non-dynamical setting. Let $\mathcal{X}$ be a compact metrizable space. Pontrjagin-Schnirelmann PS32] proved

Theorem 5.1. There exists a metric $d$ on $\mathcal{X}$ satisfying $\overline{\operatorname{dim}}_{\mathrm{M}}(\mathcal{X}, d)=$ $\operatorname{dim} \mathcal{X}$.

Compare this statement with

Theorem 5.2. There exists a metric $d$ on $\mathcal{X}$ satisfying $\underline{\operatorname{dim}}_{\mathrm{M}}(\mathcal{X}, d)=$ $\operatorname{dim} \mathcal{X}$.

They look similar. But their natures are different. A (now) standard approach to Theorem 5.2 is to use the Baire category theorem as follows. Fix an arbitrary metric $d$ on $\mathcal{X}$ and consider an infinite dimensional Banach space $(V,\|\cdot\|)$. We denote by $C(\mathcal{X}, V)$ the space of continuous maps from $\mathcal{X}$ to $V$ endowed with the norm topology. For each $n \geq 1$ we consider $A_{n} \subset C(\mathcal{X}, V)$ consisting of $f: \mathcal{X} \rightarrow V$ such that $f$ is a $(1 / n)$-embedding with respect to $d$ and satisfies

$$
\exists \varepsilon<1 / n: \frac{\log \#(f(\mathcal{X}),\|\cdot\|, \varepsilon)}{\log (1 / \varepsilon)}<\operatorname{dim} \mathcal{X}+\frac{1}{n} .
$$

It is not hard to show that $A_{n}$ are open and dense 17 . Then $\bigcap_{n=1}^{\infty} A_{n}$ is a residual (i.e. dense and $G_{\delta}$ ) subset of $C(\mathcal{X}, V)$ (in particular, nonempty). On the other hand this is equal to

$$
\left\{f \in C(\mathcal{X}, V) \mid f \text { is an embedding and } \underline{\operatorname{dim}}_{M}(f(\mathcal{X}),\|\cdot\|)=\operatorname{dim} \mathcal{X}\right\} .
$$

Pick $f$ in this set. Then the metric $\|f(x)-f(y)\|(x, y \in \mathcal{X})$ has the lower Minkowski dimension equal to $\operatorname{dim} \mathcal{X}$. This proves Theorem 5.2 .

\footnotetext{
${ }^{16}$ Indeed we cannot find this statement in PS32]. The main theorem of their paper states that $\operatorname{dim} \mathcal{X}$ is equal to the infimum of $\underline{\operatorname{dim}}_{\mathrm{M}}(\mathcal{X}, d)$ over $d \in \mathscr{D}(\mathcal{X})$. But their argument actually proves Theorem 5.1.

17 "Open" is easy. To show "dense", take arbitrary $f \in C(\mathcal{X}, V)$ and $\delta>0$. Choose $0<\varepsilon<1 / n$ such that $d(x, y)<\varepsilon$ implies $\|f(x)-f(y)\|<\delta$. There exists an $\varepsilon$-embedding $\pi: \mathcal{X} \rightarrow P$ in a simplicial complex $P$ of dimension $\leq \operatorname{dim} \mathcal{X}$. From Lemma 5.3 (2) and (3) in $\$ 5.2$ we can find a linear embedding $g: P \rightarrow V$ with $\|g(\pi(x))-f(x)\|<\delta$. From Lemma $5.3(1), \log \#\left(g(P),\|\cdot\|, \varepsilon^{\prime}\right) / \log \left(1 / \varepsilon^{\prime}\right)$ is less than $\operatorname{dim} \mathcal{X}+1 / n$ for sufficiently small $\varepsilon^{\prime}$. This shows $g \circ \pi \in A_{n}$. So $A_{n}$ is dense. Therefore the main point of the proof of Theorem 5.2 is a "polyhedral approximation". The basic idea of the proof of Theorem 5.1 is also a polyhedral approximation, but in a much more accurate way. See $\$ 5.3$.
} 
Let's try a similar approach to Theorem 5.1. It is natural to consider (5.1)

$$
\left\{f \in C(\mathcal{X}, V) \mid f \text { is an embedding and } \overline{\operatorname{dim}}_{\mathrm{M}}(f(\mathcal{X}),\|\cdot\|)=\operatorname{dim} \mathcal{X}\right\} .
$$

One might hope that this is also a residual subset of $C(\mathcal{X}, V)$. But this does not hold true. We can prove that if $\mathcal{X}$ is an infinite set then

$$
\left\{f \in C(\mathcal{X}, V) \mid \overline{\operatorname{dim}}_{\mathrm{M}}(f(\mathcal{X}),\|\cdot\|)=\infty\right\}
$$

is a residual subset of $C(\mathcal{X}, V)$. This implies that the set (5.1) is never residual in a nontrivial situation (namely if $\mathcal{X}$ is infinite and $\operatorname{dim} \mathcal{X}<\infty)$. It is a very thin set. So it is more delicate to find an element in (5.1). We note that the set (5.1) is however dense; see $\$ 5.3$.

5.2. Preparations on combinatorial topology. As we promised in 92.1. all simplicial complexes are assumed to have only finitely many vertices.

Let $P$ be a simplicial complex. We denote the set of vertices of $P$ by $\operatorname{Ver}(P)$. For $v \in \operatorname{Ver}(P)$ we define the open star $O_{P}(v)$ as the union of the open simplices of $P$ one of whose vertex is $v$. (We declare that $\{v\}$ itself is an open simplex.) The open star $O_{P}(v)$ is an open neighborhood of $v$ and $\left\{O_{P}(v)\right\}_{v \in \operatorname{Ver}(P)}$ is an open cover of $P$. When vertices $v_{0}, \ldots, v_{n}$ span a simplex $\Delta$ in $P$, we set $O_{P}(\Delta)=\bigcup_{i=0}^{n} O_{P}\left(v_{i}\right)$.

Let $P$ and $Q$ be simplicial complexes. A map $f: P \rightarrow Q$ is said to be simplicial if it satisfies the following two conditions:

- For every simplex $\Delta \subset P, f(\Delta)$ is a simplex in $Q$. (In particular $f(v) \in \operatorname{Ver}(Q)$ for every $v \in \operatorname{Ver}(P)$.)

- If $v_{0}, \ldots, v_{n} \in \operatorname{Ver}(P)$ span a simplex in $P$ then

$$
f\left(\sum_{i=0}^{n} \lambda_{i} v_{i}\right)=\sum_{i=0}^{n} \lambda_{i} f\left(v_{i}\right),
$$

where $0 \leq \lambda_{i} \leq 1$ and $\sum_{i=0}^{n} \lambda_{i}=1$.

Let $V$ be a vector space over real numbers. A map $f: P \rightarrow V$ is said to be linear if for any $v_{0}, \ldots, v_{n} \in \operatorname{Ver}(P)$ spanning a simplex in $P$

$$
f\left(\sum_{i=0}^{n} \lambda_{i} v_{i}\right)=\sum_{i=0}^{n} \lambda_{i} f\left(v_{i}\right),
$$

where $0 \leq \lambda_{i} \leq 1$ and $\sum_{i=0}^{n} \lambda_{i}=1$. We denote the space of linear maps $f: P \rightarrow V$ by $\operatorname{Hom}(P, V)$.

Lemma 5.3. Let $(V,\|\cdot\|)$ be a Banach space and $P$ a simplicial complex. 
(1) If $f: P \rightarrow V$ is a linear map with $\operatorname{diam} f(P) \leq 2$, then for any $0<\varepsilon \leq 1$

$$
\#(f(P),\|\cdot\|, \varepsilon) \leq C(P) \cdot(1 / \varepsilon)^{\operatorname{dim} P},
$$

where $C(P)$ is a positive constant depending only on $\operatorname{dim} P$ and the number of simplices of $P$.

(2) Suppose $V$ is infinite dimensional. Then the set

$$
\{f \in \operatorname{Hom}(P, V) \mid f \text { is injective }\}
$$

is dense in $\operatorname{Hom}(P, V)$. (Indeed it is also open; but we do not need this.) Here $\operatorname{Hom}(P, V) \cong V^{\operatorname{Ver}(P)}$ is endowed with the product topology.

(3) Let $(\mathcal{X}, d)$ be a compact metric space and $\varepsilon, \delta>0$. Let $\pi: \mathcal{X} \rightarrow$ $P$ be a continuous map satisfying diam $\pi^{-1}\left(O_{P}(v)\right)<\varepsilon$ for all $v \in \operatorname{Ver}(P)$. Let $f: \mathcal{X} \rightarrow V$ be a continuous map such that

$$
d(x, y)<\varepsilon \Longrightarrow\|f(x)-f(y)\|<\delta .
$$

Then there exists a linear map $g: P \rightarrow V$ satisfying

$$
\|f(x)-g(\pi(x))\|<\delta \quad(x \in \mathcal{X}) .
$$

Moreover if $f(\mathcal{X}) \subset B_{1}^{\circ}(V)$ (the open unit ball) then it can be chosen so that $g(P) \subset B_{1}^{\circ}(V)$.

Proof. (1) We can assume that $P$ is a simplex (we denote its vertices by $\left.v_{0}, \ldots, v_{n}\right)$ and that $f\left(v_{0}\right)=0$ and $\left\|f\left(v_{i}\right)\right\| \leq 2$. Set

$$
\Delta=\left\{\left(\lambda_{1}, \ldots, \lambda_{n}\right) \in[0,1]^{n} \mid \lambda_{1}+\cdots+\lambda_{n} \leq 1\right\} .
$$

Then $f(P)$ is covered by the $(\varepsilon / 3)$-open balls

$$
B_{\varepsilon / 3}^{\circ}\left(\lambda_{1} f\left(v_{1}\right)+\cdots+\lambda_{n} f\left(v_{n}\right)\right)
$$

where

$$
\left(\lambda_{1}, \ldots, \lambda_{n}\right) \in \Delta \cap\left(\frac{\varepsilon}{6 n} \mathbb{Z}\right)^{n} .
$$

(2) Let $\operatorname{Ver}(P)=\left\{v_{0}, \ldots, v_{n}\right\}$. The set (5.2) contains

$$
\left\{f \in \operatorname{Hom}(P, V) \mid f\left(v_{0}\right), \ldots, f\left(v_{n}\right) \text { are affinely independent }\right\},
$$

which is dense because $V$ is infinite dimensional.

(3) Let $v \in \operatorname{Ver}(P)$. Pick $x_{v} \in \pi^{-1}\left(O_{P}(v)\right)$ and set $g(v)=f\left(x_{v}\right)$. If $\pi^{-1}\left(O_{P}(v)\right)=\emptyset$ then $g(v)$ may be an arbitrary point in $B_{1}^{\circ}(V)$. We extend $g$ to a linear map $g: P \rightarrow V$. Let $x \in \mathcal{X}$ and $\Delta^{\circ}$ be the open simplex of $P$ containing $\pi(x)$. Let $v_{0}, \ldots, v_{n}$ be the vertices of $\Delta^{\circ}$. Then $\pi(x)=\sum_{i=0}^{n} \lambda_{i} v_{i}$ with $0<\lambda_{i} \leq 1$ and $\sum_{i=0}^{n} \lambda_{i}=1$. $g(\pi(x))=\sum_{i=0}^{n} \lambda_{i} f\left(x_{v_{i}}\right)$. 
Since $\pi(x) \in O_{P}\left(v_{i}\right), x \in \pi^{-1}\left(O_{P}\left(v_{i}\right)\right)$ and hence $d\left(x, x_{v_{i}}\right)<\varepsilon$. Then $\left\|f(x)-f\left(x_{v_{i}}\right)\right\|<\delta$. It follows that

$$
\|f(x)-g(\pi(x))\| \leq \sum_{i=0}^{n} \lambda_{i}\left\|f(x)-f\left(x_{v_{i}}\right)\right\|<\delta .
$$

If $f(\mathcal{X}) \subset B_{1}^{\circ}(V)$ then $g(v) \in B_{1}^{\circ}(V)$ for all $v \in \operatorname{Ver}(P)$ and hence $g(P) \subset B_{1}^{\circ}(V)$.

Let $f: \mathcal{X} \rightarrow P$ be a continuous map from a topological space $\mathcal{X}$ to a simplicial complex $P$. It is said to be essential if for any $v_{0}, \ldots, v_{n} \in$ $\operatorname{Ver}(P)$ spanning a simplex in $P$

$$
f^{-1}\left(O_{P}\left(v_{0}\right) \cap \cdots \cap O_{P}\left(v_{n}\right)\right) \neq \emptyset .
$$

Lemma 5.4. Let $f: \mathcal{X} \rightarrow P$ be a continuous map from a topological space $\mathcal{X}$ to a simplicial complex $P$. There exists a subcomplex $P^{\prime} \subset P$ such that $f(\mathcal{X}) \subset P^{\prime}$ and $f: \mathcal{X} \rightarrow P^{\prime}$ is essential.

Proof. It is easy to check that $f: \mathcal{X} \rightarrow P$ is essential if and only if $f(\mathcal{X}) \not \subset P^{\prime}$ for any proper subcomplex $P^{\prime} \subset P$. Then the statement is trivial; just take the minimal subcomplex $P^{\prime} \subset P$ containing $f(\mathcal{X})$.

The next lemma is one of the central ingredients of the proof Theorem 3.12. It has a spirit similar to Lemma 5.3 (3), though its statement is rather technical (see Corollary 5.7 for a simplified version). Its rough idea is as follows: Let $\mathcal{X}$ be a topological space and $P, Q$ simplicial complexes. Let $\pi: \mathcal{X} \rightarrow P$ and $q: \mathcal{X} \rightarrow Q$ be continuous maps. We would like to formulate a condition which guarantees the existence of a simplicial map $h: P \rightarrow Q$ such that $h \circ \pi$ is approximately equal to $q$.

For two open covers $\mathcal{U}$ and $\mathcal{V}$ of $\mathcal{X}$, we denote by $\mathcal{U} \prec \mathcal{V}$ if $\mathcal{V}$ is a refinement of $\mathcal{U}$, namely for every $V \in \mathcal{V}$ there exists $U \in \mathcal{U}$ satisfying $V \subset U$.

Lemma 5.5. Let $\mathcal{X}$ be a topological space and $P, Q$ simplicial complexes. Let $\pi: \mathcal{X} \rightarrow P$ and $q_{i}: \mathcal{X} \rightarrow Q, 1 \leq i \leq N$, be continuous maps. We suppose that $\pi: \mathcal{X} \rightarrow P$ is essential and satisfies

$$
\left\{q_{i}^{-1}\left(O_{Q}(w)\right)\right\}_{w \in \operatorname{Ver}(Q)} \prec\left\{\pi^{-1}\left(O_{P}(v)\right)\right\}_{v \in \operatorname{Ver}(P)}
$$

for every $1 \leq i \leq N$. (Here both sides are open covers of $\mathcal{X}$.) Then there exist simplicial maps $h_{i}: P \rightarrow Q, 1 \leq i \leq N$, such that

(1) For every $1 \leq i \leq N$ and $x \in \mathcal{X}$ the two points $q_{i}(x)$ and $h_{i} \circ \pi(x)$ belong to the same simplex of $Q$.

(2) Let $1 \leq i \leq N$. Let $\Delta \subset P$ be a simplex and $Q^{\prime} \subset Q$ a subcomplex. If $\pi^{-1}\left(O_{P}(\Delta)\right) \subset q_{i}^{-1}\left(Q^{\prime}\right)$ then $h_{i}(\Delta) \subset Q^{\prime}$. 
(3) For $1 \leq i<j \leq N$ and a simplex $\Delta \subset P$, if $q_{i}=q_{j}$ on $\pi^{-1}\left(O_{P}(\Delta)\right)$ then $h_{i}=h_{j}$ on $\Delta$.

Proof. Let $v \in \operatorname{Ver}(P)$. We can choose $h_{i}(v) \in \operatorname{Ver}(Q), 1 \leq i \leq N$, satisfying

- $\pi^{-1}\left(O_{P}(v)\right) \subset q_{i}^{-1}\left(O_{Q}\left(h_{i}(v)\right)\right)$.

- If $q_{i}=q_{j}$ on $\pi^{-1}\left(O_{P}(v)\right)$ then $h_{i}(v)=h_{j}(v)$.

Suppose $v_{0}, \ldots, v_{n} \in \operatorname{Ver}(P)$ span a simplex in $P$. Since $\pi$ is essential,

$$
\begin{aligned}
\emptyset & \neq \pi^{-1}\left(O_{P}\left(v_{0}\right) \cap \cdots \cap O_{P}\left(v_{n}\right)\right) \\
& \subset q_{i}^{-1}\left(O_{Q}\left(h_{i}\left(v_{0}\right)\right) \cap \cdots \cap O_{Q}\left(h_{i}\left(v_{n}\right)\right)\right) .
\end{aligned}
$$

In particular $O_{Q}\left(h_{i}\left(v_{0}\right)\right) \cap \cdots \cap O_{Q}\left(h_{i}\left(v_{n}\right)\right) \neq \emptyset$. So $h_{i}\left(v_{0}\right), \ldots, h_{i}\left(v_{n}\right)$ span a simplex in $Q$. This implies that we can extend $h_{i}$ to a simplicial map from $P$ to $Q$. The condition (3) immediately follows from the choices of $h_{i}(v)$.

For the proof of (1), take $x \in \mathcal{X}$ and let $v_{0}, \ldots, v_{n} \in \operatorname{Ver}(P)$ be the vertices of the open simplex of $P$ containing $\pi(x)$. Then $h_{i}(\pi(x))$ belongs to the simplex spanned by $h_{i}\left(v_{0}\right), \ldots, h_{i}\left(v_{n}\right)$. On the other hand

$$
\begin{aligned}
x & \in \pi^{-1}\left(O_{P}\left(v_{0}\right) \cap \cdots \cap O_{P}\left(v_{n}\right)\right) \\
& \subset q_{i}^{-1}\left(O_{Q}\left(h_{i}\left(v_{0}\right)\right) \cap \cdots \cap O_{Q}\left(h_{i}\left(v_{n}\right)\right)\right) .
\end{aligned}
$$

Hence $q_{i}(x) \in O_{Q}\left(h_{i}\left(v_{0}\right)\right) \cap \cdots \cap O_{Q}\left(h_{i}\left(v_{n}\right)\right)$ and there exists a simplex $\Delta \subset Q$ containing $q_{i}(x)$ and $h_{i}\left(v_{0}\right), \ldots, h_{i}\left(v_{n}\right)$. Then $\Delta$ contains both $q_{i}(x)$ and $h_{i}(\pi(x))$.

For the proof of (2), take a subcomplex $Q^{\prime} \subset Q$. Then

\section{Claim 5.6.}

Let $v \in \operatorname{Ver}(P)$ and $1 \leq i \leq N$. If $\pi^{-1}\left(O_{P}(v)\right) \subset q_{i}^{-1}\left(Q^{\prime}\right)$ then $h_{i}(v) \in Q^{\prime}$ and $\pi^{-1}\left(O_{P}(v)\right) \subset q_{i}^{-1}\left(O_{Q^{\prime}}\left(h_{i}(v)\right)\right)$.

Proof.

$$
q_{i}\left(\pi^{-1}\left(O_{P}(v)\right)\right) \subset Q^{\prime} \cap O_{Q}\left(h_{i}(v)\right) .
$$

If $h_{i}(v) \notin Q^{\prime}$ then the right-hand side is empty. So $h_{i}(v) \in Q^{\prime}$ and hence $Q^{\prime} \cap O_{Q}\left(h_{i}(v)\right)=O_{Q^{\prime}}\left(h_{i}(v)\right)$.

Let $\Delta \subset P$ be a simplex with vertices $v_{0}, \ldots, v_{n}$. If $\pi^{-1}\left(O_{P}(\Delta)\right) \subset$ $q_{i}^{-1}\left(Q^{\prime}\right)$ then $h_{i}\left(v_{0}\right), \ldots, h_{i}\left(v_{n}\right) \in Q^{\prime}$ and (since $\pi$ is essential)

$$
\begin{aligned}
& \emptyset \neq \pi^{-1}\left(O_{P}\left(v_{0}\right) \cap \cdots \cap O_{P}\left(v_{n}\right)\right) \\
& \quad \subset q_{i}^{-1}\left(O_{Q^{\prime}}\left(h_{i}\left(v_{0}\right)\right) \cap \cdots \cap O_{Q^{\prime}}\left(h_{i}\left(v_{n}\right)\right)\right) .
\end{aligned}
$$

In particular $O_{Q^{\prime}}\left(h_{i}\left(v_{0}\right)\right) \cap \cdots \cap O_{Q^{\prime}}\left(h_{i}\left(v_{n}\right)\right) \neq \emptyset$. So $h_{i}\left(v_{0}\right), \ldots, h_{i}\left(v_{n}\right)$ span a simplex in $Q^{\prime}$. Then $h_{i}(\Delta) \subset Q^{\prime}$. 
Letting $N=1$ in Lemma 5.5, we get the following corollary. This is used in $\$ 5.3$.

Corollary 5.7. Let $\mathcal{X}$ be a topological space and $P, Q$ simplicial complexes. Let $\pi: \mathcal{X} \rightarrow P$ and $q: \mathcal{X} \rightarrow Q$ be continuous maps. If $\pi$ is essential and

$$
\left\{q^{-1}\left(O_{Q}(w)\right)\right\}_{w \in \operatorname{Ver}(Q)} \prec\left\{\pi^{-1}\left(O_{P}(v)\right)\right\}_{v \in \operatorname{Ver}(P)},
$$

then there exists a simplicial map $h: P \rightarrow Q$ such that for every $x \in \mathcal{X}$ the two points $q(x)$ and $h(\pi(x))$ belong to the same simplex in $Q$.

We need to introduce a notation for Lebesgue number. Let $(\mathcal{X}, d)$ be a compact metric space and $\mathcal{U}$ its open cover. We denote the Lebesgue number of $\mathcal{U}$ by $L N(\mathcal{X}, d, \mathcal{U})$, namely it is the supremum of $\varepsilon>0$ such that if a subset $A \subset \mathcal{X}$ satisfies $\operatorname{diam} A<\varepsilon$ then there exists $U \in \mathcal{U}$ containing $A$.

5.3. Warmup: the proof of Pontrjagin-Schnirelmann's theorem. Here we prove Pontrjagin-Schnirelmann's theorem (Theorem 5.1) by using the preparations of $\$ 5.2$. This is a toy model of the proof of Theorem 3.12. (This subsection is logically independent of the proof of Theorem 3.12.) Our proof of Theorem 5.1 roughly follows the line of ideas of [PS32]. Our purpose here is to help readers to get acquainted with how to use lemmas in the previous subsection. Theorem 5.1 follows from

Theorem 5.8. Let $(V,\|\cdot\|)$ be an infinite dimensional Banach space and $\mathcal{X}$ a compact metrizable space. For a dense subset of $f$ in $C(\mathcal{X}, V)$ (the space of continuous maps from $\mathcal{X}$ to $V$ endowed with the norm topology), $f$ is a topological embedding and satisfies

$$
\overline{\operatorname{dim}}_{\mathrm{M}}(f(\mathcal{X}),\|\cdot\|)=\operatorname{dim} \mathcal{X} .
$$

Proof. We can assume that $D:=\operatorname{dim} \mathcal{X}<\infty$. Fix a metric $d$ on $\mathcal{X}$ and take arbitrary $f \in C(\mathcal{X}, V)$ and $\eta>0$. We want to construct a topological embedding $f^{\prime}: \mathcal{X} \rightarrow V$ satisfying $\left\|f(x)-f^{\prime}(x)\right\|<\eta$ and $\overline{\operatorname{dim}}_{\mathrm{M}}\left(f^{\prime}(\mathcal{X}),\|\cdot\|\right)=D$. (It is enough to prove $\overline{\operatorname{dim}}_{\mathrm{M}}(f(\mathcal{X}),\|\cdot\|) \leq D$ because Minkowski dimension always dominates topological dimension.) We may assume that $f(\mathcal{X})$ is contained in the open unit ball $B_{1}^{\circ}(V)$. We will inductively construct the following data $(n \geq 1)$ :

Data 5.9. (1) Positive numbers $\varepsilon_{n}$ and $\delta_{n}$ with $\varepsilon_{n+1}<\varepsilon_{n} / 2, \delta_{n+1}<$ $\delta_{n} / 2$ and $\delta_{1}<\eta / 2$.

(2) A $(1 / n)$-embedding $\pi_{n}:(\mathcal{X}, d) \rightarrow P_{n}$ such that $P_{n}$ is a simplicial complex of dimension $\leq D$. 
(3) A linear embedding $g_{n}: P_{n} \rightarrow B_{1}^{\circ}(V)$.

We assume the following conditions:

Condition 5.10.

$$
\#\left(g_{n}\left(P_{n}\right),\|\cdot\|, \varepsilon\right)< \begin{cases}\left(\frac{2}{\varepsilon}\right)^{D+\frac{1}{n-1}} & \left(\varepsilon<\varepsilon_{n-1}\right) \\ \left(\frac{1}{\varepsilon}\right)^{D+\frac{1}{n}} & \left(\varepsilon<\varepsilon_{n}\right) .\end{cases}
$$

Here the former condition is empty for $n=1$.

(2) Set $f_{n}=g_{n} \circ \pi_{n}: \mathcal{X} \rightarrow V$. If a continuous map $f^{\prime}: \mathcal{X} \rightarrow V$ satisfies $\left\|f^{\prime}(x)-f_{n}(x)\right\|<\delta_{n}$ then $f^{\prime}$ is a $(1 / n)$-embedding with respect to $d$.

$$
\begin{gathered}
\left\|f_{1}(x)-f(x)\right\|<\frac{\eta}{2}, \\
\left\|f_{n}(x)-f_{n+1}(x)\right\|<\min \left(\frac{\varepsilon_{n}}{8}, \frac{\delta_{n}}{2}\right) .
\end{gathered}
$$

Suppose we have constructed the above data. Then we can define $f^{\prime} \in C(\mathcal{X}, V)$ by $f^{\prime}(x)=\lim _{n \rightarrow \infty} f_{n}(x)$. It follows from Condition 5.10 (3) that $\left\|f^{\prime}(x)-f_{n}(x)\right\|<\delta_{n}$ for all $n \geq 1$. So by Condition 5.10 (2) $f^{\prime}$ is a $(1 / n)$-embedding for all $n \geq 1$, which implies that $f^{\prime}$ is a topological embedding. It also satisfies $\left\|f^{\prime}(x)-f(x)\right\|<\eta$.

We want to prove $\overline{\operatorname{dim}}_{\mathrm{M}}\left(f^{\prime}(\mathcal{X}),\|\cdot\|\right) \leq D$. Let $0<\varepsilon<\varepsilon_{1}$. Take $n>1$ with $\varepsilon_{n} \leq \varepsilon<\varepsilon_{n-1}$. It follows from Condition 5.10 (3) that $\left\|f^{\prime}(x)-f_{n}(x)\right\|<\varepsilon_{n} / 4$. Hence

$$
\begin{aligned}
\#\left(f^{\prime}(\mathcal{X}),\|\cdot\|, \varepsilon\right) & \leq \#\left(f_{n}(\mathcal{X}),\|\cdot\|, \varepsilon-\frac{\varepsilon_{n}}{2}\right) \\
& \leq \#\left(g_{n}\left(P_{n}\right),\|\cdot\|, \varepsilon-\frac{\varepsilon_{n}}{2}\right) \quad\left(\text { by } f_{n}(\mathcal{X}) \subset g_{n}\left(P_{n}\right)\right) \\
& \left.<\left(\frac{2}{\varepsilon-\frac{\varepsilon_{n}}{2}}\right)^{D+\frac{1}{n-1}} \quad \text { (by Condition 5.10 }(1)\right) \\
& \leq\left(\frac{4}{\varepsilon}\right)^{D+\frac{1}{n-1}} \quad\left(\text { by } \varepsilon \geq \varepsilon_{n}\right) .
\end{aligned}
$$

Since $n \rightarrow \infty$ as $\varepsilon \rightarrow 0$, this shows $\overline{\operatorname{dim}}_{\mathrm{M}}\left(f^{\prime}(\mathcal{X}),\|\cdot\|\right) \leq D$.

Now we start to construct the data. We choose $0<\tau_{1}<1$ so that

$$
d(x, y)<\tau_{1} \Longrightarrow\|f(x)-f(y)\|<\eta / 2 .
$$

Let $\pi_{1}:(\mathcal{X}, d) \rightarrow P_{1}$ be a $\tau_{1}$-embedding in a simplicial complex $P_{1}$ with $\operatorname{dim} P_{1} \leq D$. (Since $D=\operatorname{dim} \mathcal{X}=\lim _{\varepsilon \rightarrow 0} \operatorname{Widim}_{\varepsilon}(\mathcal{X}, d)$, we can find such a map.) By subdividing $P_{1}$ sufficiently fine, we can assume $\operatorname{diam} \pi_{1}^{-1}\left(O_{P_{1}}(v)\right)<\tau_{1}$ for all $v \in \operatorname{Ver}\left(P_{1}\right)$. Then by Lemma 5.3 (3) we 
can find a linear map $\tilde{g}_{1}: P_{1} \rightarrow B_{1}^{\circ}(V)$ satisfying $\left\|\tilde{g}_{1}\left(\pi_{1}(x)\right)-f(x)\right\|<$ $\eta / 2$. Since linear embeddings are dense in $\operatorname{Hom}\left(P_{1}, V\right)$ (Lemma 5.3 $(2)$ ), we can also find a linear embedding $g_{1}: P_{1} \rightarrow B_{1}^{\circ}(V)$ satisfying $\left\|g_{1}\left(\pi_{1}(x)\right)-f(x)\right\|<\eta / 2$. By Lemma [5.3 (1), we can find $\varepsilon_{1}>0$ satisfying Condition 5.10 (1). The map $f_{1}=g_{1} \circ \pi_{1}$ is a 1-embedding and "1-embedding" is an open condition. So there exists $\delta_{1}>0$ such that Condition 5.10 (2) holds true. This finishes the construction for $n=1$.

Suppose that we have already done the construction for the $n$-th step. We try to construct the data for the $(n+1)$-th step. We subdivide $P_{n}$ sufficiently fine so that every simplex $\Delta \subset P_{n}$ satisfies $\operatorname{diam}\left(g_{n}(\Delta),\|\cdot\|\right)<\min \left(\varepsilon_{n} / 8, \delta_{n} / 2\right)$. We take $0<\tau_{n+1}<1 /(n+1)$ with

$$
\tau_{n+1}<L N\left(\mathcal{X}, d,\left\{\pi_{n}^{-1}\left(O_{P_{n}}(v)\right)\right\}_{v \in \operatorname{Ver}\left(P_{n}\right)}\right) .
$$

Take a $\tau_{n+1}$-embedding $\pi_{n+1}:(\mathcal{X}, d) \rightarrow P_{n+1}$ with a simplicial complex $P_{n+1}$ of dimension $\leq D$. By subdividing $P_{n+1}$ sufficiently fine, we can assume $\operatorname{diam}\left(\pi_{n+1}^{-1}\left(O_{P_{n+1}}(v)\right)\right)<\tau_{n+1}$ for all $v \in \operatorname{Ver}\left(P_{n+1}\right)$. Moreover, by replacing $P_{n+1}$ with a subcomplex (if necessarily), we can assume that $\pi_{n+1}: \mathcal{X} \rightarrow P_{n+1}$ is essential (Lemma 5.4). The open cover $\left\{\pi_{n+1}^{-1}\left(O_{P_{n+1}}(v)\right)\right\}_{v \in \operatorname{Ver}\left(P_{n+1}\right)}$ of $\mathcal{X}$ becomes a refinement of $\left\{\pi_{n}^{-1}\left(O_{P_{n}}(v)\right)\right\}_{v \in \operatorname{Ver}\left(P_{n}\right)}$ because of the Lebesgue number condition above. Then by applying Corollary 5.7 to $\pi_{n+1}: \mathcal{X} \rightarrow P_{n+1}$ and $\pi_{n}: \mathcal{X} \rightarrow P_{n}$ (with $P=P_{n+1}$ and $Q=P_{n}$ ), we can find a simplicial map $h: P_{n+1} \rightarrow P_{n}$ such that for every $x \in \mathcal{X}$ the two points $\pi_{n}(x)$ and $h\left(\pi_{n+1}(x)\right)$ belong to the same simplex of $P_{n}$. Set $\tilde{g}_{n+1}=g_{n} \circ h$ : $P_{n+1} \rightarrow B_{1}^{\circ}(V)$. This satisfies (recall $f_{n}=g_{n} \circ \pi_{n}$ )

$$
\left\|\tilde{g}_{n+1}\left(\pi_{n+1}(x)\right)-f_{n}(x)\right\|<\min \left(\frac{\varepsilon_{n}}{8}, \frac{\delta_{n}}{2}\right) .
$$

Since $\tilde{g}_{n+1}\left(P_{n+1}\right) \subset g_{n}\left(P_{n}\right)$, the induction hypothesis implies

$$
\#\left(\tilde{g}_{n+1}\left(P_{n+1}\right),\|\cdot\|, \varepsilon\right)<\left(\frac{1}{\varepsilon}\right)^{D+\frac{1}{n}} \quad\left(\varepsilon<\varepsilon_{n}\right) .
$$

By Lemma 5.3 (1), there exists $0<\varepsilon_{n+1}<\varepsilon_{n} / 2$ such that for all linear maps $g: P_{n+1} \rightarrow V$ with $g\left(P_{n+1}\right) \subset B_{1}^{\circ}(V)$

$$
\#\left(g\left(P_{n+1}\right),\|\cdot\|, \varepsilon\right)<\left(\frac{1}{\varepsilon}\right)^{D+\frac{1}{n+1}} \quad\left(\varepsilon<\varepsilon_{n+1}\right) .
$$


We slightly perturb $\tilde{g}_{n+1}$ by Lemma 5.3 (2): There exists a linear embedding $g_{n+1}: P_{n+1} \rightarrow B_{1}^{\circ}(V)$ such that

$$
\begin{gathered}
\left\|g_{n+1}\left(\pi_{n+1}(x)\right)-f_{n}(x)\right\|<\min \left(\frac{\varepsilon_{n}}{8}, \frac{\delta_{n}}{2}\right), \\
\left\|g_{n+1}(u)-\tilde{g}_{n+1}(u)\right\|<\frac{\varepsilon_{n+1}}{4} \quad\left(u \in P_{n+1}\right) .
\end{gathered}
$$

By the choice of $\varepsilon_{n+1}$ we have

$$
\#\left(g_{n+1}\left(P_{n+1}\right),\|\cdot\|, \varepsilon\right)<\left(\frac{1}{\varepsilon}\right)^{D+\frac{1}{n+1}} \quad\left(\varepsilon<\varepsilon_{n+1}\right) .
$$

For $\varepsilon_{n+1} \leq \varepsilon<\varepsilon_{n}$

$$
\begin{aligned}
\#\left(g_{n+1}\left(P_{n+1}\right),\|\cdot\|, \varepsilon\right) & \leq \#\left(\tilde{g}_{n+1}\left(P_{n+1}\right),\|\cdot\|, \varepsilon-\frac{\varepsilon_{n+1}}{2}\right) \quad(\text { by (15.4) }) \\
& \leq \#\left(\tilde{g}_{n+1}\left(P_{n+1}\right),\|\cdot\|, \frac{\varepsilon}{2}\right) \quad\left(\text { by } \varepsilon-\frac{\varepsilon_{n+1}}{2} \geq \frac{\varepsilon}{2}\right) \\
& <\left(\frac{2}{\varepsilon}\right)^{D+\frac{1}{n}} \quad(\text { by }(\underline{5.3})) .
\end{aligned}
$$

$f_{n+1}=g_{n+1} \circ \pi_{n+1}$ is a $1 /(n+1)$-embedding. So we can find $0<$ $\delta_{n+1}<\delta_{n} / 2$ satisfying Condition 5.10 (2). This has completed the construction for the $(n+1)$-th step.

5.4. Dynamical tiling construction. Here we review a construction introduced in [GLT16]. Let $(\mathcal{X}, T)$ be a dynamical system and $\varphi: \mathcal{X} \rightarrow$ $[0,1]$ a continuous function. For $x \in \mathcal{X}$ we consider

$$
\left\{\left(a, \frac{1}{\varphi\left(T^{a} x\right)}\right) \mid a \in \mathbb{Z} \text { with } \varphi\left(T^{a} x\right)>0\right\} \subset \mathbb{R}^{2} .
$$

We assume that this is nonempty for every $x \in \mathcal{X}$. (Namely, for every $x \in \mathcal{X}$, there exists $a \in \mathbb{Z}$ with $\varphi\left(T^{a} x\right)>0$.) Let $\mathbb{R}^{2}=\bigcup_{a \in \mathbb{Z}} V_{\varphi}(x, a)$ be the associated Voronoi diagram, namely $V_{\varphi}(x, a)$ is the set of $u \in \mathbb{R}^{2}$ satisfying

$$
\left|u-\left(a, \frac{1}{\varphi\left(T^{a} x\right)}\right)\right| \leq\left|u-\left(b, \frac{1}{\varphi\left(T^{b} x\right)}\right)\right|
$$

for any $b \in \mathbb{Z}$ with $\varphi\left(T^{b} x\right)>0$. This is a convex subset of the plane. We set

$$
I_{\varphi}(x, a)=V_{\varphi}(x, a) \cap(\mathbb{R} \times\{0\}) .
$$

See Figure 5.1. If $\varphi\left(T^{a} x\right)=0$ then $V_{\varphi}(x, a)=I_{\varphi}(x, a)=\emptyset$. 


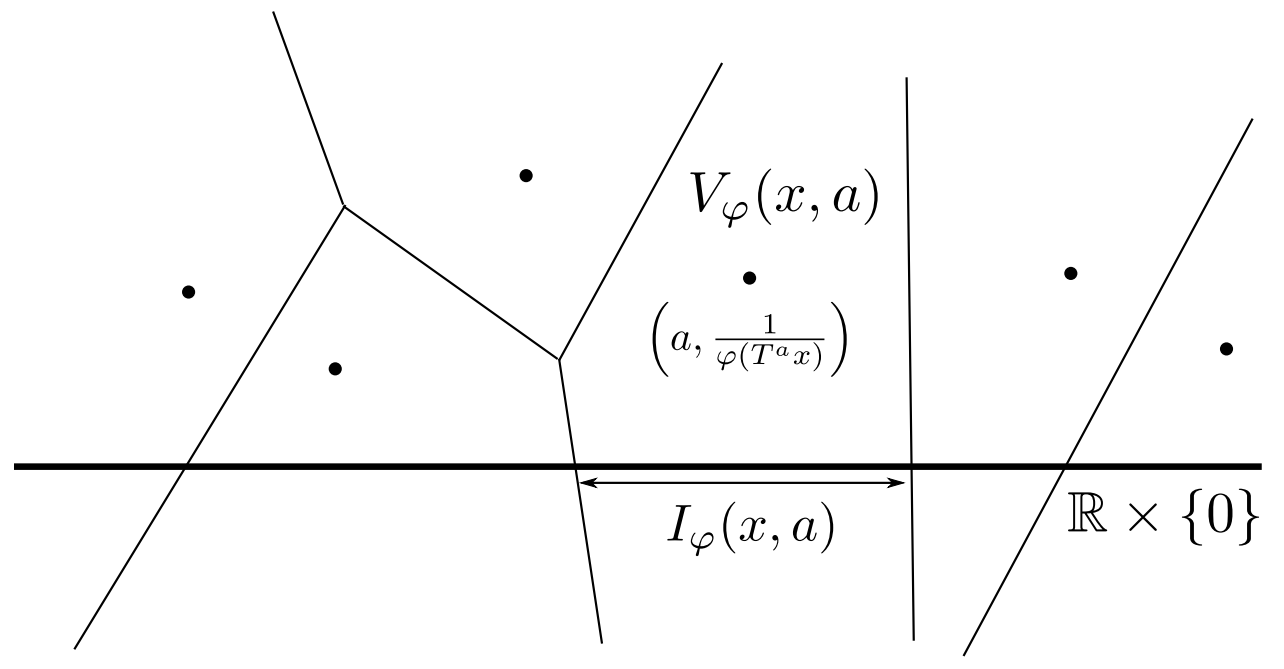

Figure 5.1. Dynamical tiling construction

We naturally identify $\mathbb{R} \times\{0\}$ with $\mathbb{R}$. Then this construction gives a decomposition of $\mathbb{R}$ :

$$
\mathbb{R}=\bigcup_{a \in \mathbb{Z}} I_{\varphi}(x, a)
$$

$I_{\varphi}(x, a)$ are closed intervals. We set

$$
\partial_{\varphi}(x)=\bigcup_{a \in \mathbb{Z}} \partial I_{\varphi}(x, a) \subset \mathbb{R}
$$

where $\partial I_{\varphi}(x, a)$ is the boundary of the interval $I_{\varphi}(x, a)$ (e.g. $\partial[0,1]=$ $\{0,1\})$. This construction is equivariant, namely

$$
I_{\varphi}\left(T^{n} x, a\right)=-n+I_{\varphi}(x, a+n), \quad \partial_{\varphi}\left(T^{n} x\right)=-n+\partial_{\varphi}(x) .
$$

Lemma 5.11. Suppose $(\mathcal{X}, T)$ has the marker property. Then for any $\varepsilon>0$ we can find a continuous function $\varphi: \mathcal{X} \rightarrow[0,1]$ such that (5.5) is nonempty for every $x \in \mathcal{X}$ and that it satisfies the following conditions.

(1) There exists $M>0$ such that $I_{\varphi}(x, a) \subset(a-M, a+M)$ for all $x \in \mathcal{X}$ and $a \in \mathbb{Z}$ (in particular, all $I_{\varphi}(x, a)$ are finite length intervals).

$$
\lim _{R \rightarrow \infty} \frac{\sup _{x \in \mathcal{X}}\left|\partial_{\varphi}(x) \cap[0, R]\right|}{R}<\varepsilon .
$$

Here $\left|\partial_{\varphi}(x) \cap[0, R]\right|$ is the cardinality of $\partial_{\varphi}(x) \cap[0, R]$. Notice that the above (1) implies that $\partial_{\varphi}(x)$ is a discrete set in the real line. 
(3) The intervals $I_{\varphi}(x, a)$ continuously depend on $x \in \mathcal{X}$ : i.e. if $x_{k} \rightarrow x$ in $\mathcal{X}$ and $I_{\varphi}(x, a)$ has positive length then $I_{\varphi}\left(x_{k}, a\right)$ converges to $I_{\varphi}(x, a)$ in the Hausdorff topology, and if $I_{\varphi}(x, a)=$ $\emptyset$ then for all $k$ large enough $I_{\varphi}\left(x_{k}, a\right)$ is also empty.

Proof. Take $N>1 / \varepsilon$. From the marker property, there exists an open set $U \subset \mathcal{X}$ such that $U \cap T^{-n} U=\emptyset$ for $1 \leq n \leq N$ and $\mathcal{X}=\bigcup_{n \in \mathbb{Z}} T^{-n} U$. We can find $M>N$ and a compact subset $K \subset U$ with $\mathcal{X}=\bigcup_{n=0}^{M-1} T^{-n} K$. Let $\varphi: \mathcal{X} \rightarrow[0,1]$ be a continuous function such that $\varphi=1$ on $K$ and $\operatorname{supp} \varphi \subset U$.

Let $x \in \mathcal{X}$ and consider

$$
\Lambda_{x}=\left\{a \in \mathbb{Z} \mid \varphi\left(T^{a} x\right)>0\right\}, \quad \Lambda_{x}^{\prime}=\left\{a \in \mathbb{Z} \mid \varphi\left(T^{a} x\right)=1\right\} .
$$

Any gap of $\Lambda_{x}$ (i.e. the difference between two successive numbers in $\Lambda_{x}$ ) is larger than $N$, and any gap of $\Lambda_{x}^{\prime}$ is smaller than or equal to $M$. For $a \in \Lambda_{x}^{\prime}$ the interval $I_{\varphi}(x, a)$ contains $a$ as an interior point.

Let $a \in \Lambda_{x}$. There exist $s, t \in \Lambda_{x}^{\prime}$ such that $s<a<t$ and $a-s, t-a \leq$ $M$. Then $I_{\varphi}(x, a) \subset(s, t) \subset(a-M, a+M)$. The continuity of $I_{\varphi}(x, a)$ is an immediate consequence of the definition. The condition (2) follows from

$$
\lim _{R \rightarrow \infty} \frac{\sup _{x \in \mathcal{X}}\left|\partial_{\varphi}(x) \cap[0, R]\right|}{R} \leq \lim _{R \rightarrow \infty} \frac{\sup _{x \in \mathcal{X}}\left|\Lambda_{x} \cap[0, R]\right|}{R} \leq \frac{1}{N}<\varepsilon .
$$

5.5. Proof of Theorem 3.12. Let $(V,\|\cdot\|)$ be an infinite dimensional Banach space and $(\mathcal{X}, T)$ a dynamical system. As in $\$ 5.3$ we denote by $C(\mathcal{X}, V)$ the space of continuous maps from $\mathcal{X}$ to $V$ endowed with the norm topology. Theorem 3.12 follows from

Theorem 5.12. Suppose $(\mathcal{X}, T)$ has the marker property. For a dense subset of $f \in C(\mathcal{X}, V), f$ is a topological embedding and satisfies

$$
\overline{\operatorname{mdim}}_{\mathrm{M}}\left(\mathcal{X}, T, f^{*}\|\cdot\|\right)=\operatorname{mdim}(\mathcal{X}, T) .
$$

Here $f^{*}\|\cdot\|$ is the metric $\|f(x)-f(y)\|(x, y \in \mathcal{X})$.

Proof. We can assume $D:=\operatorname{mdim}(\mathcal{X}, T)<\infty$. Fix a metric $d$ on $\mathcal{X}$ and take an arbitrary $f \in C(\mathcal{X}, V)$ and $\eta>0$. We want to construct a topological embedding $f^{\prime}: \mathcal{X} \rightarrow V$ satisfying $\left\|f(x)-f^{\prime}(x)\right\|<\eta$ for all $x \in \mathcal{X}$ and $\overline{\operatorname{mdim}}_{\mathrm{M}}\left(\mathcal{X}, T,\left(f^{\prime}\right)^{*}\|\cdot\|\right)=D$. (It is enough to show $\overline{\operatorname{mdim}}_{\mathrm{M}}\left(\mathcal{X}, T,\left(f^{\prime}\right)^{*}\|\cdot\|\right) \leq D$ because the reverse inequality is always true by Theorem 2.2$)$. We may assume that $f(\mathcal{X})$ is contained in the open unit ball $B_{1}^{\circ}(V)$. 
We prepare some notations. For a natural number $N$ we set $[N]=$ $\{0,1,2, \ldots, N-1\}$. We define a norm $\|\cdot\|_{N}$ on $V^{N}$ (the $N$-th power of $V)$ by

$$
\left\|\left(x_{0}, x_{1}, \ldots, x_{N-1}\right)\right\|_{N}=\max \left(\left\|x_{0}\right\|,\left\|x_{1}\right\|, \ldots,\left\|x_{N-1}\right\|\right) .
$$

For a simplicial complex $P$ we define the number $A(P)$ as the minimum of $A \geq 1$ such that for any linear map $g: P \rightarrow B_{1}(V)$ we have $\#(g(P),\|\cdot\|, \varepsilon) \leq(1 / \varepsilon)^{A}$ for all $0<\varepsilon \leq 1 / 2$. (Such a number always exists by Lemma $5.3(1)$.) For simplicial complexes $P$ and $Q$, we denote their join by $P * Q$, namely it is the quotient of $[0,1] \times P \times Q$ by the equivalence relation

$$
(0, p, q) \sim\left(0, p, q^{\prime}\right), \quad(1, p, q) \sim\left(1, p^{\prime}, q\right), \quad\left(p, p^{\prime} \in P, q, q^{\prime} \in Q\right) .
$$

We denote the equivalence class of $(t, p, q)$ by $(1-t) p \oplus t q$. We identify $P$ and $Q$ with $\{(0, p, *) \mid p \in P\}$ and $\{(1, *, q) \mid q \in Q\}$ in $P * Q$ respectively. If $g: P \rightarrow B_{1}(V)$ and $g^{\prime}: Q \rightarrow B_{1}(V)$ we define the map $g * g^{\prime}: P * Q \rightarrow$ $B_{1}(V)$ by sending $(1-t) p \oplus t q$ to $(1-t) g(p)+t g^{\prime}(q)$.

For a continuous map $f^{\prime}: \mathcal{X} \rightarrow V$ and $I \subset \mathbb{R}$ we define $\Phi_{f^{\prime}, I}: \mathcal{X} \rightarrow$ $V^{I \cap \mathbb{Z}}$ by

$$
\Phi_{f^{\prime}, I}(x)=\left(f^{\prime}\left(T^{a} x\right)\right)_{a \in I \cap \mathbb{Z}} .
$$

For a natural number $R$ we set $\Phi_{f^{\prime}, R}:=\Phi_{f^{\prime},[0, R)}: \mathcal{X} \rightarrow V^{R}$. We fix a continuous function $\alpha: \mathbb{R} \rightarrow[0,1]$ such that $\alpha(t)=1$ for $t \leq 1 / 2$ and $\alpha(t)=0$ for $t \geq 3 / 4$.

We will inductively construct the following data for $n \geq 1$.

Data 5.13. (1) $1 / 2>\varepsilon_{1}>\varepsilon_{2}>\cdots>0$ with $\varepsilon_{n+1}<\varepsilon_{n} / 2$ and $\eta / 2>\delta_{1}>\delta_{2}>\cdots>0$ with $\delta_{n+1}<\delta_{n} / 2$.

(2) A natural number $N_{n}$.

(3) A continuous function $\varphi_{n}: \mathcal{X} \rightarrow[0,1]$ such that for every $x \in \mathcal{X}$ there exists $a \in \mathbb{Z}$ with $\varphi_{n}\left(T^{a} x\right)>0$. We apply the dynamical tiling construction of $\$ 5.4$ to this function and get the decomposition $\mathbb{R}=\bigcup_{a \in \mathbb{Z}} I_{\varphi_{n}}(x, a)$ for each $x \in \mathcal{X}$.

(4) A $(1 / n)$-embedding $\pi_{n}:\left(\mathcal{X}, d_{N_{n}}\right) \rightarrow P_{n}$ with a simplicial complex $P_{n}$ of dimension less than $\left(D+\frac{1}{n}\right) N_{n}$.

(5) A $(1 / n)$-embedding $\pi_{n}^{\prime}:(\mathcal{X}, d) \rightarrow Q_{n}$ with a simplicial complex $Q_{n}$.

(6) For each $\lambda \in\left[N_{n}\right]$, a linear embedding $g_{n, \lambda}: P_{n} \rightarrow B_{1}^{\circ}(V)$.

(7) A linear embedding $g_{n}^{\prime}: Q_{n} \rightarrow B_{1}^{\circ}(V)$.

We assume the following conditions. 
Condition 5.14. (1) For each $\lambda \in\left[N_{n}\right]$, the join $g_{n, \lambda} * g_{n}^{\prime}: P_{n} *$ $Q_{n} \rightarrow B_{1}^{\circ}(V)$ is a linear embedding. For $\lambda_{1} \neq \lambda_{2}$

$$
g_{n, \lambda_{1}} * g_{n}^{\prime}\left(P_{n} * Q_{n}\right) \cap g_{n, \lambda_{2}} * g_{n}^{\prime}\left(P_{n} * Q_{n}\right)=g_{n}^{\prime}\left(Q_{n}\right) .
$$

(2) Set $g_{n}=\left(g_{n, 0}, g_{n, 1}, \ldots, g_{n, N_{n}-1}\right): P_{n} \rightarrow V^{N_{n}}$. Then

$$
\#\left(g_{n}\left(P_{n}\right),\|\cdot\|_{N_{n}}, \varepsilon\right)<\left\{\begin{array}{ll}
4^{N_{n}}\left(\frac{2}{\varepsilon}\right)^{\left(D+\frac{2}{n-1}\right) N_{n}} & \left(0<\varepsilon<\varepsilon_{n-1}\right) \\
\left(\frac{1}{\varepsilon}\right)^{\left(D+\frac{1}{n}\right) N_{n}} & \left(0<\varepsilon<\varepsilon_{n}\right)
\end{array} .\right.
$$

Here the former condition is empty for $n=1$.

(3) There exists $M_{n}>0$ such that $I_{\varphi_{n}}(x, a) \subset\left(a-M_{n}, a+M_{n}\right)$ for all $x \in \mathcal{X}$ and $a \in \mathbb{Z}$. The sets $\partial_{\varphi_{n}}(x)$ are discrete in $\mathbb{R}$ and satisfy

$$
\lim _{R \rightarrow \infty} \frac{\sup _{x \in \mathcal{X}}\left|\partial_{\varphi_{n}}(x) \cap[0, R]\right|}{R}<\frac{1}{2 n N_{n}^{2} \cdot A\left(P_{n} * Q_{n}\right)} .
$$

(4) We define a continuous map $f_{n}: \mathcal{X} \rightarrow B_{1}^{\circ}(V)$ as follows: Let $x \in \mathcal{X}$ and take $a \in \mathbb{Z}$ with $0 \in I_{\varphi_{n}}(x, a)$. Take $b \in \mathbb{Z}$ such that $b \equiv a\left(\bmod N_{n}\right)$ and $0 \in b+\left[N_{n}\right]$. Set

$$
\begin{aligned}
f_{n}(x)= & \left\{1-\alpha\left(\operatorname{dist}\left(0, \partial_{\varphi_{n}}(x)\right)\right)\right\} g_{n,-b}\left(\pi_{n}\left(T^{b} x\right)\right) \\
& +\alpha\left(\operatorname{dist}\left(0, \partial_{\varphi_{n}}(x)\right)\right) g_{n}^{\prime}\left(\pi_{n}^{\prime}(x)\right),
\end{aligned}
$$

where $\operatorname{dist}\left(0, \partial_{\varphi_{n}}(x)\right):=\min _{t \in \partial_{\varphi_{n}}(x)}|t|$. Then we assume that if a continuous map $f^{\prime}: \mathcal{X} \rightarrow V$ satisfies $\left\|f^{\prime}(x)-f_{n}(x)\right\|<\delta_{n}$ for all $x \in \mathcal{X}$ then it is a $(1 / n)$-embedding with respect to $d$.

(5) For all $x \in \mathcal{X}$

$$
\begin{aligned}
\left\|f(x)-f_{1}(x)\right\| & <\frac{\eta}{2} \\
\left\|f_{n}(x)-f_{n+1}(x)\right\| & <\min \left(\frac{\varepsilon_{n}}{8}, \frac{\delta_{n}}{2}\right) .
\end{aligned}
$$

Suppose we have constructed the above data. Then we can define $f^{\prime} \in C(\mathcal{X}, V)$ by $f^{\prime}(x)=\lim _{n \rightarrow \infty} f_{n}(x)$. It satisfies $\left\|f^{\prime}(x)-f(x)\right\|<\eta$ and $\left\|f^{\prime}(x)-f_{n}(x)\right\|<\min \left(\varepsilon_{n} / 4, \delta_{n}\right)$ for all $n \geq 1$. From Condition $5.14(4), f^{\prime}$ is a $(1 / n)$-embedding for all $n \geq 1$. So it is a topological embedding. Set $d^{\prime}(x, y)=\left\|f^{\prime}(x)-f^{\prime}(y)\right\|$. We want to show $\overline{\operatorname{mdim}}_{\mathrm{M}}\left(\mathcal{X}, T, d^{\prime}\right) \leq D$. Notice that $\overline{\operatorname{mdim}}_{\mathrm{M}}\left(\mathcal{X}, T, d^{\prime}\right)$ is equal to

$$
\limsup _{\varepsilon \rightarrow 0}\left\{\lim _{R \rightarrow \infty}\left(\frac{\log \#\left(\Phi_{f^{\prime}, R}(\mathcal{X}),\|\cdot\|_{R}, \varepsilon\right)}{R}\right) / \log (1 / \varepsilon)\right\} .
$$


Claim 5.15. Let $0<\varepsilon<\varepsilon_{n-1}(n \geq 2)$. For sufficiently large natural numbers $R$

$$
\#\left(\Phi_{f_{n}, R}(\mathcal{X}),\|\cdot\|_{R}, \varepsilon\right)<2^{4 R}\left(\frac{2}{\varepsilon}\right)^{\left(D+\frac{2}{n-1}\right) R+\frac{R}{n}} .
$$

Proof. In this proof $n \geq 2$ is fixed and $R$ is a sufficiently large natural number. Let $x \in \mathcal{X}$. We call $J=\left\{b, b+1, \ldots, b+N_{n}-1\right\}(b \in \mathbb{Z})$ good for $x$ if there is $a \in \mathbb{Z}$ such that $b \equiv a\left(\bmod N_{n}\right)$ and $\left(b-1, b+N_{n}\right) \subset$ $I_{\varphi_{n}}(x, a)$. If $J$ is good for $x$ then $\Phi_{f_{n}, J}(x)$ is contained in $g_{n}\left(P_{n}\right)$ in $V^{N_{n}}$. We denote by $\mathcal{J}_{x}$ the union of $J \subset[R]$ which are good for $x$. The number of possibilities of $\mathcal{J}_{x}$ (when $x \in \mathcal{X}$ varies) is bounded by $2^{R}$. Then \# $\left(\Phi_{f_{n}, R}(\mathcal{X}),\|\cdot\|_{R}, \varepsilon\right)$ is bounded by

$$
2^{R} \cdot \underbrace{\#\left(g_{n}\left(P_{n}\right),\|\cdot\|_{N_{n}}, \varepsilon\right)^{\frac{R}{N_{n}}}}_{\text {contribution over } \mathcal{J}_{x}} \cdot \underbrace{\#\left(f_{n}(\mathcal{X}),\|\cdot\|, \varepsilon\right)^{2 N_{n} \sup _{x \in \mathcal{X}}\left|\partial_{\varphi_{n}}(x) \cap[0, R]\right|+2 N_{n}}}_{\text {contribution over }[0, R) \backslash \mathcal{J}_{x}} .
$$

Here " $+2 N_{n}$ " is the edge effect. $f_{n}(\mathcal{X})$ is contained in the union of $g_{n, \lambda} * g_{n}^{\prime}\left(P_{n} * Q_{n}\right)$ over $\lambda \in\left[N_{n}\right]$. So

$$
\#\left(f_{n}(\mathcal{X}),\|\cdot\|, \varepsilon\right) \leq N_{n}\left(\frac{1}{\varepsilon}\right)^{A\left(P_{n} * Q_{n}\right)} .
$$

Using Condition $5.14(2)$ and (3), we get the statement of the claim.

We now return to the proof of Theorem 5.12 .

Let $0<\varepsilon<\varepsilon_{1}$. Take $n>1$ with $\varepsilon_{n} \leq \varepsilon<\varepsilon_{n-1}$. Recall that $\left\|f^{\prime}(x)-f_{n}(x)\right\|<\varepsilon_{n} / 4$. Hence

$$
\begin{aligned}
\#\left(\Phi_{f^{\prime}, R}(\mathcal{X}),\|\cdot\|_{R}, \varepsilon\right) & \leq \#\left(\Phi_{f_{n}, R}(\mathcal{X}),\|\cdot\|_{R}, \varepsilon-\frac{\varepsilon_{n}}{2}\right) \\
& \leq \#\left(\Phi_{f_{n}, R}(\mathcal{X}),\|\cdot\|_{R}, \frac{\varepsilon}{2}\right) .
\end{aligned}
$$

From Claim 5.15,

$$
\lim _{R \rightarrow \infty} \frac{\log \#\left(\Phi_{f^{\prime}, R}(\mathcal{X}),\|\cdot\|_{R}, \varepsilon\right)}{R} \leq 4+\left(D+\frac{2}{n-1}+\frac{1}{n}\right) \log (4 / \varepsilon) .
$$

Notice that $n \rightarrow \infty$ as $\varepsilon \rightarrow 0$. Using (5.6) we get $\overline{\operatorname{mdim}}_{\mathrm{M}}\left(\mathcal{X}, T, d^{\prime}\right) \leq D$.

Induction: Step 1. Now we start to construct the data. Take $0<\tau_{1}<1$ such that

$$
d(x, y)<\tau_{1} \Longrightarrow\|f(x)-f(y)\|<\frac{\eta}{2} .
$$

From the definition of mean dimension, we can find $N_{1}>0$ and $\tau_{1}$ embeddings $\pi_{1}:\left(\mathcal{X}, d_{N_{1}}\right) \rightarrow P_{1}$ and $\pi_{1}^{\prime}:(\mathcal{X}, d) \rightarrow Q_{1}$ such that $P_{1}$ and 
$Q_{1}$ are simplicial complexes with $\operatorname{dim} P_{1}<N_{1}(D+1)$. By subdividing $P_{1}$ and $Q_{1}$, we can assume that

$$
\operatorname{diam}\left(\pi_{1}^{-1}\left(O_{P_{1}}(v)\right), d_{N_{1}}\right)<\tau_{1}, \quad \operatorname{diam}\left(\left(\pi_{1}^{\prime}\right)^{-1}\left(O_{Q_{1}}(w)\right), d\right)<\tau_{1}
$$

for all $v \in \operatorname{Ver}\left(P_{1}\right)$ and $w \in \operatorname{Ver}\left(Q_{1}\right)$. By Lemma 5.3 (3), we can find linear maps $\tilde{g}_{1, \lambda}: P_{1} \rightarrow B_{1}^{\circ}(V)$ for $\lambda \in\left[N_{1}\right]$ and $\tilde{g}_{1}^{\prime}: Q_{1} \rightarrow B_{1}^{\circ}(V)$ satisfying

$$
\left\|f\left(T^{\lambda} x\right)-\tilde{g}_{1, \lambda}\left(\pi_{1}(x)\right)\right\|<\frac{\eta}{2}, \quad\left\|f(x)-\tilde{g}_{1}^{\prime}\left(\pi_{1}^{\prime}(x)\right)\right\|<\frac{\eta}{2}
$$

for all $x \in \mathcal{X}$. By Lemma $5.3(2)$, we can replace $\tilde{g}_{1, \lambda}$ and $\tilde{g}_{1}^{\prime}$ with linear embeddings $g_{1, \lambda}: P_{1} \rightarrow B_{1}^{\circ}(V)$ and $g_{1}^{\prime}: Q_{1} \rightarrow B_{1}^{\circ}(V)$ satisfying Condition 5.14 (1) and

$$
\left\|f\left(T^{\lambda} x\right)-g_{1, \lambda}\left(\pi_{1}(x)\right)\right\|<\frac{\eta}{2}, \quad\left\|f(x)-g_{1}^{\prime}\left(\pi_{1}^{\prime}(x)\right)\right\|<\frac{\eta}{2} .
$$

By Lemma 5.3 (1), we can find $0<\varepsilon_{1}<1 / 2$ satisfying Condition 5.14 (2).

By Lemma 5.11, we can take a continuous function $\varphi_{1}: \mathcal{X} \rightarrow[0,1]$ satisfying Condition 5.14 (3). By Condition 5.14 (1) (which has been already established for $n=1$ ), the map $f_{1}: \mathcal{X} \rightarrow V$ becomes a 1embedding. It also satisfies Condition 5.14 (5) by (5.7). Since "1embedding" is an open condition, we can find $0<\delta_{1}<\eta / 2$ satisfying Condition 5.14 (4). The first step of the induction has been completed.

Induction: Step $n \Rightarrow$ Step $n+1$. Next we suppose that we have constructed the date for $n$. We will construct the date for $n+1$.

We subdivide $P_{n} * Q_{n}$ sufficiently fine so that

$$
\operatorname{diam}\left(g_{n, \lambda} * g_{n}^{\prime}(\Delta),\|\cdot\|\right)<\min \left(\frac{\varepsilon_{n}}{8}, \frac{\delta_{n}}{2}\right)
$$

for any simplex $\Delta \subset P_{n} * Q_{n}$ and $\lambda \in\left[N_{n}\right]$.

We define a continuous map $q_{n}: \mathcal{X} \rightarrow P_{n} * Q_{n}$ as follows. Let $x \in \mathcal{X}$ and take $a \in \mathbb{Z}$ with $0 \in I_{\varphi_{n}}(x, a)$. We take $b \in \mathbb{Z}$ such that $b \equiv a\left(\bmod N_{n}\right)$ and $0 \in b+\left[N_{n}\right]$. Set

$$
q_{n}(x)=\left\{1-\alpha\left(\operatorname{dist}\left(0, \partial_{\varphi_{n}}(x)\right)\right)\right\} \pi_{n}\left(T^{b} x\right) \oplus \alpha\left(\operatorname{dist}\left(0, \partial_{\varphi_{n}}(x)\right)\right) \pi_{n}^{\prime}(x) .
$$

We take $0<\tau_{n+1}<1 /(n+1)$ such that

- If $d(x, y)<\tau_{n+1}$ then $\left\|f_{n}(x)-f_{n}(y)\right\|<\min \left(\varepsilon_{n} / 8, \delta_{n} / 2\right)$ and

$$
\left|\operatorname{dist}\left(0, \partial_{\varphi_{n}}(x)\right)-\operatorname{dist}\left(0, \partial_{\varphi_{n}}(y)\right)\right|<\frac{1}{4} \text {. }
$$

- If $d(x, y)<\tau_{n+1}$ and $(-1 / 4,1 / 4) \subset I_{\varphi_{n}}(x, a)$ then $I_{\varphi_{n}}(y, a)$ contains 0 as an interior point. 
- $\tau_{n+1}$ is smaller than the Lebesgue number of the open cover $\left\{q_{n}^{-1}\left(O_{P_{n} * Q_{n}}(v)\right)\right\}_{v \in \operatorname{Ver}\left(P_{n} * Q_{n}\right)}$ :

$$
\tau_{n+1}<L N\left(\mathcal{X}, d,\left\{q_{n}^{-1}\left(O_{P_{n} * Q_{n}}(v)\right)\right\}_{v \in \operatorname{Ver}\left(P_{n} * Q_{n}\right)}\right) .
$$

Take a $\tau_{n+1}$-embedding $\pi_{n+1}^{\prime}:(\mathcal{X}, d) \rightarrow Q_{n+1}$ with a simplicial complex $Q_{n+1}$. We can assume $\operatorname{diam}\left(\left(\pi_{n+1}^{\prime}\right)^{-1}\left(O_{Q_{n+1}}(w)\right), d\right)<\tau_{n+1}$ for every $w \in \operatorname{Ver}\left(Q_{n+1}\right)$. By Lemma $5.3(3)$, we can take a linear map $\tilde{g}_{n+1}^{\prime}: Q_{n+1} \rightarrow B_{1}^{\circ}(V)$ satisfying

$$
\left\|\tilde{g}_{n+1}^{\prime}\left(\pi_{n+1}^{\prime}(x)\right)-f_{n}(x)\right\|<\min \left(\frac{\varepsilon_{n}}{8}, \frac{\delta_{n}}{2}\right) .
$$

We can find $N_{n+1}>N_{n}$ such that

- There exists a $\tau_{n+1}$-embedding $\pi_{n+1}:\left(\mathcal{X}, d_{N_{n+1}}\right) \rightarrow P_{n+1}$ with a simplicial complex $P_{n+1}$ of dimension less than $N_{n+1}\left(D+\frac{1}{n+1}\right)$.

$$
1+\sup _{x \in \mathcal{X}}\left|\partial_{\varphi_{n}}(x) \cap\left[0, N_{n+1}\right]\right|<\frac{N_{n+1}}{2 n N_{n}^{2} \cdot A\left(P_{n} * Q_{n}\right)} .
$$

Here we have used Condition $5.14(3) 18$ for $\varphi_{n}$.

By subdividing $P_{n+1}$ sufficiently fine, we assume that for any two simplexes $\Delta, \Delta^{\prime} \subset P_{n+1}$ with $\Delta \cap \Delta^{\prime} \neq \emptyset$

$$
\operatorname{diam}\left(\pi_{n+1}^{-1}\left(O_{P_{n+1}}(\Delta)\right) \cup \pi_{n+1}^{-1}\left(O_{P_{n+1}}\left(\Delta^{\prime}\right)\right), d_{N_{n+1}}\right)<\tau_{n+1} .
$$

Moreover by Lemma 5.4 we can assume that $\pi_{n+1}: \mathcal{X} \rightarrow P_{n+1}$ is essential.

We apply Lemma 5.5 (with $P=P_{n+1}, Q=P_{n} * Q_{n}, N=N_{n+1}$, and $Q^{\prime}=P_{n}$ or $\left.Q_{n}\right)$ to continuous maps $\pi_{n+1}: \mathcal{X} \rightarrow P_{n+1}$ and $q_{n} \circ T^{\lambda}: \mathcal{X} \rightarrow$ $P_{n} * Q_{n}, \lambda \in\left[N_{n+1}\right]$. (The assumption of Lemma 5.5 is satisfied because of the above Lebesgue number condition.) Then we get simplicial maps $h_{\lambda}: P_{n+1} \rightarrow P_{n} * Q_{n}, \lambda \in\left[N_{n+1}\right]$, so that

- For every $\lambda \in\left[N_{n+1}\right]$ and $x \in \mathcal{X}$, the two points $h_{\lambda}\left(\pi_{n+1}(x)\right)$ and $q_{n}\left(T^{\lambda} x\right)$ belong to the same simplex of $P_{n} * Q_{n}$.

- Let $\Delta \subset P_{n+1}$ be a simplex and $\lambda \in\left[N_{n+1}\right]$. If $\pi_{n+1}^{-1}\left(O_{P_{n+1}}(\Delta)\right) \subset$ $T^{-\lambda} q_{n}^{-1}\left(P_{n}\right)$ then $h_{\lambda}(\Delta) \subset P_{n}$. Similarly, if $\pi_{n+1}^{-1}\left(O_{P_{n+1}}(\Delta)\right) \subset$ $T^{-\lambda} q_{n}^{-1}\left(Q_{n}\right)$, then $h_{\lambda}(\Delta) \subset Q_{n}$.

- For $\lambda, \lambda^{\prime} \in\left[N_{n+1}\right]$ and a simplex $\Delta \subset P_{n+1}$, if $q_{n} \circ T^{\lambda}=q_{n} \circ T^{\lambda^{\prime}}$ on $\pi_{n+1}^{-1}\left(O_{P_{n+1}}(\Delta)\right)$ then $h_{\lambda}=h_{\lambda^{\prime}}$ on $\Delta$.

\footnotetext{
${ }^{18}$ Here is a technical point. The number $A\left(P_{n} * Q_{n}\right)$ is defined by using the simplicial complex structure of $P_{n} * Q_{n}$. We use the natural simplicial complex structure of the join $P_{n} * Q_{n}$ here, not its subdivision introduced in (5.8).
} 
Let $u \in P_{n+1}$, and let $\Delta \subset P_{n+1}$ be a simplex containing $u$. Since $\pi_{n+1}: \mathcal{X} \rightarrow P_{n+1}$ is essential, there exists $x \in \pi_{n+1}^{-1}\left(O_{P_{n+1}}(\Delta)\right)$. Let $\lambda \in\left[N_{n+1}\right]$. We take $a, b \in \mathbb{Z}$ such that $\lambda \in I_{\varphi_{n}}(x, a), b \equiv a\left(\bmod N_{n}\right)$ and $\lambda \in b+\left[N_{n}\right]$. We set

$$
\tilde{g}_{n+1, \lambda}(u)=g_{n, \lambda-b} * g_{n}^{\prime}\left(h_{\lambda}(u)\right) \in B_{1}^{\circ}(V) .
$$

We will check that this is independent of the choices of $x$ and $a$; see Claim 5.16 below. In this setting we have $f_{n}\left(T^{\lambda} x\right)=g_{n, \lambda-b} *$ $g_{n}^{\prime}\left(q_{n}\left(T^{\lambda} x\right)\right)$. It follows from (5.8) and the first condition of $h_{\lambda}$ that

$$
\left\|\tilde{g}_{n+1, \lambda}\left(\pi_{n+1}(x)\right)-f_{n}\left(T^{\lambda} x\right)\right\|<\min \left(\frac{\varepsilon_{n}}{8}, \frac{\delta_{n}}{2}\right) .
$$

Claim 5.16. The map $\tilde{g}_{n+1, \lambda}: P_{n+1} \rightarrow V$ is a linear map.

Proof. The point is that the above definition of $\tilde{g}_{n+1, \lambda}(u)$ is independent of the choices of $x$ and $a$. Let $\Delta^{\prime} \subset P_{n+1}$ be another simplex containing $u$ and pick $x^{\prime} \in \pi_{n+1}^{-1}\left(O_{P_{n+1}}\left(\Delta^{\prime}\right)\right)$. We take $a^{\prime}, b^{\prime} \in \mathbb{Z}$ such that $\lambda \in$ $I_{\varphi_{n}}\left(x^{\prime}, a^{\prime}\right), b^{\prime} \equiv a^{\prime}\left(\bmod N_{n}\right)$ and $\lambda \in b^{\prime}+\left[N_{n}\right]$.

Case 1: Suppose $\operatorname{dist}\left(\lambda, \partial_{\partial_{n}}(x)\right)>1 / 4$. We have $d\left(T^{\lambda} x, T^{\lambda} x^{\prime}\right)<$ $\tau_{n+1}$ by (5.11). From the second condition of the choice of $\tau_{n+1}$, we have $a=a^{\prime}$ and $b=b^{\prime}$. Hence

$$
g_{n, \lambda-b} * g_{n}^{\prime}\left(h_{\lambda}(u)\right)=g_{n, \lambda-b^{\prime}} * g_{n}^{\prime}\left(h_{\lambda}(u)\right) .
$$

Case 2: Suppose $\operatorname{dist}\left(\lambda, \partial_{\varphi_{n}}(x)\right) \leq 1 / 4$. Take an arbitrary $y \in$ $\pi_{n+1}^{-1}\left(O_{P_{n+1}}(\Delta)\right) \cup \pi_{n+1}^{-1}\left(O_{P_{n+1}}\left(\Delta^{\prime}\right)\right)$. We have $d\left(T^{\lambda} x, T^{\lambda} y\right)<\tau_{n+1}$ by (5.11) and hence dist $\left(\lambda, \partial_{\varphi_{n}}(y)\right)<1 / 2$ by the condition (5.9) of the choice of $\tau_{n+1}$. Then $q_{n}\left(T^{\lambda} y\right)=\pi_{n}^{\prime}\left(T^{\lambda} y\right) \in Q_{n}$. Hence $\pi_{n+1}^{-1}\left(O_{P_{n+1}}(\Delta)\right) \cup$ $\pi_{n+1}^{-1}\left(O_{P_{n+1}}\left(\Delta^{\prime}\right)\right) \subset T^{-\lambda} q_{n}^{-1}\left(Q_{n}\right)$. So $h_{\lambda}(\Delta) \cup h_{\lambda}\left(\Delta^{\prime}\right) \subset Q_{n}$ by the second condition of $h_{\lambda}$. This implies

$$
g_{n, \lambda-b} * g_{n}^{\prime}\left(h_{\lambda}(u)\right)=g_{n}^{\prime}\left(h_{\lambda}(u)\right)=g_{n, \lambda-b^{\prime}} * g_{n}^{\prime}\left(h_{\lambda}(u)\right) .
$$

Claim 5.17. Set $\tilde{g}_{n+1}(u)=\left(\tilde{g}_{n+1,0}(u), \tilde{g}_{n+1,1}(u), \ldots, \tilde{g}_{n+1, N_{n+1}-1}(u)\right)$. Then for $0<\varepsilon<\varepsilon_{n}$

$$
\#\left(\tilde{g}_{n+1}\left(P_{n+1}\right),\|\cdot\|_{N_{n+1}}, \varepsilon\right)<4^{N_{n+1}}\left(\frac{1}{\varepsilon}\right)^{\left(D+\frac{2}{n}\right) N_{n+1}} .
$$

Proof. This is similar to the proof of Claim 5.15. Let $\Delta \subset P_{n+1}$ be a simplex. For $b \in \mathbb{Z} \cap\left[0, N_{n+1}-N_{n}\right]$, a discrete interval $J=$ $\left\{b, b+1, \ldots, b+N_{n}-1\right\}$ is said to be good for $\Delta$ if there exist $x \in \pi_{n+1}^{-1}\left(O_{P_{n+1}}(\Delta)\right)$ and $a \in \mathbb{Z}$ such that $b \equiv a\left(\bmod N_{n}\right)$ and $(b-1, b+$ $\left.N_{n}\right) \subset I_{\varphi_{n}}(x, a)$. This condition implies that every $y \in \pi_{n+1}^{-1}\left(O_{P_{n+1}}(\Delta)\right)$ 
satisfies $\left(b-3 / 4, b+N_{n}-1 / 4\right) \subset I_{\varphi_{n}}(y, a)$ by (5.11) and the first and second conditions of $\tau_{n+1}$. In particular for every $\lambda \in J$ and $y \in \pi_{n+1}^{-1}\left(O_{P_{n+1}}(\Delta)\right)$

$$
q_{n}\left(T^{\lambda} y\right)=q_{n}\left(T^{b} y\right)=\pi_{n}\left(T^{b} y\right) \in P_{n} .
$$

Then the second and third conditions of $h_{\lambda}$ imply $h_{\lambda}(u)=h_{b}(u) \in P_{n}$ on $u \in \Delta$ and $\lambda \in J$. Hence for $u \in \Delta$

$$
\left(\tilde{g}_{n+1, \lambda}(u)\right)_{\lambda \in J}=g_{n}\left(h_{b}(u)\right) \in g_{n}\left(P_{n}\right) \subset V^{N_{n}} .
$$

As a conclusion, if $J$ is good for $\Delta$ then $\left(\tilde{g}_{n+1, \lambda}(u)\right)_{\lambda \in J} \in g_{n}\left(P_{n}\right)$ for all $u \in \Delta$.

Let $\mathcal{J}_{\Delta}$ be the union of $J=\left\{b, b+1, \ldots, b+N_{n}-1\right\} \subset\left[N_{n+1}\right]$ which are good for $\Delta$. The number of possibilities of $\mathcal{J}_{\Delta}$ (when $\Delta \subset$ $P_{n+1}$ varies $)$ is bounded by $2^{N_{n+1}}$. Then $\#\left(\tilde{g}_{n+1}\left(P_{n+1}\right),\|\cdot\|_{N_{n+1}}, \varepsilon\right)$ is bounded by

$$
\begin{aligned}
& 2^{N_{n+1}} \times \underbrace{\#\left(g_{n}\left(P_{n}\right),\|\cdot\|_{N_{n}}, \varepsilon\right)^{\frac{N_{n+1}}{N_{n}}}}_{\text {contribution over } \mathcal{J}_{\Delta}} \\
& \times \underbrace{\#\left(\bigcup_{\lambda \in\left[N_{n}\right]} g_{n, \lambda} * g_{n}^{\prime}\left(P_{n} * Q_{n}\right),\|\cdot\|, \varepsilon\right)^{2 N_{n} \sup _{x \in \mathcal{X}}\left|\partial_{\varphi_{n}}(x) \cap\left[0, N_{n+1}\right]\right|+2 N_{n}}}_{\text {contribution over }\left[N_{n+1}\right] \backslash \mathcal{J}_{\Delta}} .
\end{aligned}
$$

We have

$$
\#\left(\bigcup_{\lambda \in\left[N_{n}\right]} g_{n, \lambda} * g_{n}^{\prime}\left(P_{n} * Q_{n}\right),\|\cdot\|, \varepsilon\right) \leq N_{n}\left(\frac{1}{\varepsilon}\right)^{A\left(P_{n} * Q_{n}\right)} .
$$

Then we get the claim by Condition 5.14(2) and (3) for $g_{n}$ and $\varphi_{n}$.

By Lemma 5.3 (1) and $\operatorname{dim} P_{n+1}<\left(D+\frac{1}{n+1}\right) N_{n+1}$, we can take $0<\varepsilon_{n+1}<\varepsilon_{n} / 2$ such that for any linear map $g: P_{n+1} \rightarrow B_{1}^{\circ}(V)^{N_{n+1}}$

$$
\#\left(g\left(P_{n+1}\right),\|\cdot\|_{N_{n+1}}, \varepsilon\right)<\left(\frac{1}{\varepsilon}\right)^{\left(D+\frac{1}{n+1}\right) N_{n+1}} \quad\left(0<\varepsilon<\varepsilon_{n+1}\right) .
$$

By lemma 5.3 (2) and the above (5.10) and (5.12), we can find linear embeddings $g_{n+1}^{\prime}: Q_{n+1} \rightarrow B_{1}^{\circ}(V)$ and $g_{n+1, \lambda}: P_{n+1} \rightarrow B_{1}^{\circ}(V), \lambda \in$ $\left[N_{n+1}\right]$, such that they satisfy Condition 5.14 (1) and for any $x \in \mathcal{X}$ 
and $u \in P_{n+1}$

$$
\begin{gathered}
\left\|g_{n+1}^{\prime}\left(\pi_{n+1}^{\prime}(x)\right)-f_{n}(x)\right\|<\min \left(\frac{\varepsilon_{n}}{8}, \frac{\delta_{n}}{2}\right), \\
\left\|g_{n+1, \lambda}\left(\pi_{n+1}(x)\right)-f_{n}\left(T^{\lambda} x\right)\right\|<\min \left(\frac{\varepsilon_{n}}{8}, \frac{\delta_{n}}{2}\right), \\
\left\|g_{n+1, \lambda}(u)-\tilde{g}_{n+1, \lambda}(u)\right\|<\frac{\varepsilon_{n+1}}{4} .
\end{gathered}
$$

It follows from (5.14) and Claim 5.17 that for $\varepsilon_{n+1} \leq \varepsilon<\varepsilon_{n}$

$$
\begin{aligned}
\#\left(g_{n+1}\left(P_{n+1}\right),\|\cdot\|_{N_{n+1}}, \varepsilon\right) & \leq \#\left(\tilde{g}_{n+1}\left(P_{n+1}\right),\|\cdot\|_{N_{n+1}}, \varepsilon-\frac{\varepsilon_{n+1}}{2}\right) \\
& \leq \#\left(\tilde{g}_{n+1}\left(P_{n+1}\right),\|\cdot\|_{N_{n+1}}, \frac{\varepsilon}{2}\right) \\
& <4^{N_{n+1}}\left(\frac{2}{\varepsilon}\right)^{\left(D+\frac{2}{n}\right) N_{n+1}} .
\end{aligned}
$$

From the choice of $\varepsilon_{n+1}$, for $0<\varepsilon<\varepsilon_{n+1}$

$$
\#\left(g_{n+1}\left(P_{n+1}\right),\|\cdot\|_{N_{n+1}}, \varepsilon\right)<\left(\frac{1}{\varepsilon}\right)^{\left(D+\frac{1}{n+1}\right) N_{n+1}} .
$$

Hence $g_{n+1, \lambda}$ satisfy Condition 5.14 (2).

From Lemma 5.11 we can choose a continuous function $\varphi_{n+1}: \mathcal{X} \rightarrow$ $[0,1]$ satisfying Condition $5.14(3)$. From (5.13), $f_{n+1}$ satisfies Condition 5.14 (5). Since $g_{n+1, \lambda}$ and $g_{n+1}^{\prime}$ satisfy Condition 5.14 (1), $f_{n+1}$ is a $1 /(n+1)$-embedding with respect to $d$. Since "1/(n+1)-embedding" is an open condition, we can choose $\delta_{n+1}>0$ satisfying Condition 5.14 (4).

We have established all the data for the $(n+1)$-th step.

\section{EXAMPle: ALGEBRAiC ACTIONS}

We study an example in this section. Probably the example below can be more generalized (e.g. more general group actions), but we restrict ourselves to a simple case because our purpose here is just to illustrate the concepts studied in the paper. We plan to study more examples in future works.

Set $\mathbb{T}=\mathbb{R} / \mathbb{Z}$. Let $r>0$ be an integer and consider the shift $\sigma:\left(\mathbb{T}^{r}\right)^{\mathbb{Z}} \rightarrow\left(\mathbb{T}^{r}\right)^{\mathbb{Z}}$ on the alphabet $\mathbb{T}^{r}=\mathbb{R}^{r} / \mathbb{Z}^{r}$. This becomes a compact Abelian group under the component-wise addition. A subset $\mathcal{X} \subset\left(\mathbb{T}^{r}\right)^{\mathbb{Z}}$ is called an algebraic action if it is a $\sigma$-invariant closed 
subgroup 19. Equivalently Sch95, Definitions 3.7 and 4.1, Theorems 3.8 and 4.2], a subset $\mathcal{X} \subset\left(\mathbb{T}^{r}\right)^{\mathbb{Z}}$ is an algebraic action if and only if there exist a positive integer $a$ and a closed subgroup $H \subset\left(\mathbb{T}^{r}\right)^{a}$ such that

$$
\mathcal{X}=\left\{\left(x_{n}\right)_{n \in \mathbb{Z}} \in\left(\mathbb{T}^{r}\right)^{\mathbb{Z}} \mid\left(x_{n}, x_{n+1}, \ldots, x_{n+a-1}\right) \in H(\forall n \in \mathbb{Z})\right\} .
$$

We define metrics $\rho$ and $\rho_{r}$ on $\mathbb{T}$ and $\mathbb{T}^{r}$ respectively by

$$
\begin{gathered}
\rho\left(t, t^{\prime}\right)=\min _{n \in \mathbb{Z}}\left|t-t^{\prime}-n\right|, \\
\rho_{r}\left(\left(t_{1}, \ldots, t_{r}\right),\left(t_{1}^{\prime}, \ldots, t_{r}^{\prime}\right)\right)=\max _{1 \leq i \leq r} \rho\left(t_{i}, t_{i}^{\prime}\right) .
\end{gathered}
$$

We define a metric $d$ on $\left(\mathbb{T}^{r}\right)^{\mathbb{Z}}$ by

$$
d(x, y)=\sum_{n \in \mathbb{Z}} 2^{-|n|} \rho_{r}\left(x_{n}, y_{n}\right), \quad\left(x_{n}, y_{n} \in \mathbb{T}^{r}\right) .
$$

Later we will use the fact that $d$ is homogeneous, namely it is invariant under the addition

$$
d(x+z, y+z)=d(x, y), \quad\left(x, y, z \in\left(\mathbb{T}^{r}\right)^{\mathbb{Z}}\right) .
$$

For $N>0$ we denote by $\pi_{N}:\left(\mathbb{T}^{r}\right)^{\mathbb{Z}} \rightarrow\left(\mathbb{T}^{r}\right)^{N}$ the projection to the $\{0,1,2, \ldots, N-1\}$-coordinates:

$$
\pi_{N}(x)=\left(x_{0}, \ldots, x_{N-1}\right) .
$$

Let $\mathcal{X} \subset\left(\mathbb{T}^{r}\right)^{\mathbb{Z}}$ be an algebraic action. Following Gromov [Gro99, §1.9] we define the projective dimension of $\mathcal{X}$ by

$$
\operatorname{prodim}(\mathcal{X})=\lim _{N \rightarrow \infty} \frac{\operatorname{dim} \pi_{N}(\mathcal{X})}{N}
$$

Here $\operatorname{dim} \pi_{N}(\mathcal{X})$ is the topological dimension of $\pi_{N}(\mathcal{X})$. This limit always exists because $\operatorname{dim} \pi_{N}(\mathcal{X})$ is subadditive in $N$. (Note that, a priori, the projective dimension may depend on the way of the embed$\operatorname{ding} \mathcal{X} \subset\left(\mathbb{T}^{r}\right)^{\mathbb{Z}}$. So the notation $\operatorname{prodim}(\mathcal{X})$ might be misleading. But we use it for simplicity.)

Li-Liang [LL18, Theorem 4.1, Theorem 7.2] proved:

$$
\operatorname{mdim}(\mathcal{X}, \sigma)=\operatorname{mdim}_{\mathrm{M}}(\mathcal{X}, \sigma, d)=\operatorname{prodim}(\mathcal{X}) .
$$

Indeed they proved more general results. But we stick to this simple case. Since mean Hausdorff dimension is bounded between mean dimension and metric mean dimension (Proposition 3.2), we also have

$$
\operatorname{mdim}_{\mathrm{H}}(\mathcal{X}, \sigma, d)=\operatorname{prodim}(\mathcal{X}) .
$$

\footnotetext{
${ }^{19}$ Since $r$ is finite, this is more restricted than in the literatures [Sch95, LL18]. They consider automorphisms of general compact Abelian groups. But we study only the restricted class here for simplicity.
} 
The purpose of this section is to show:

Proposition 6.1. Let $\mathcal{X} \subset\left(\mathbb{T}^{r}\right)^{\mathbb{Z}}$ be an algebraic action and $\mu$ the normalized Haar measure on it $(\mu(\mathcal{X})=1)$. Then

$$
\operatorname{rdim}(\mathcal{X}, \sigma, d, \mu)=\operatorname{prodim}(\mathcal{X})
$$

Hence the rate distortion dimension with respect to the Haar measure coincides with the mean dimension, mean Hausdorff dimension and metric mean dimension.

Therefore algebraic actions provide natural examples where all the dynamical dimensions studied in this paper coincide with each other.

In the sequel, we also include the proof of (6.1) for the completeness. The idea of the proof is the same as [LL18].

The following lemma is a key estimate [LL18, Lemma 4.2].

Lemma 6.2. Let $A$ be a compact Abelian group with a metric $\boldsymbol{d}$ and $f: A \rightarrow \mathbb{T}^{r}$ a continuous homomorphism satisfying $\rho_{r}(f(x), f(y)) \leq$ $\boldsymbol{d}(x, y)$. Then for any $0<\varepsilon<1 / 4$

$$
\begin{aligned}
& \operatorname{Widim}_{\varepsilon}(A, \boldsymbol{d}) \geq \operatorname{dim} f(A), \\
& \#(A, \boldsymbol{d}, \varepsilon) \geq\left(\frac{1}{4 \varepsilon}\right)^{\operatorname{dim} f(A)} .
\end{aligned}
$$

Here $\operatorname{dim} f(A)$ is the topological dimension of $f(A)$.

Proof. We can assume that $f(A)$ is connected. (If it is not, we replace $A$ with the inverse by $f$ of the connected component of $f(A)$ through the origin.) Let $\pi: \mathbb{R}^{r} \rightarrow \mathbb{T}^{r}$ be the natural covering map and set $V=\pi^{-1}(f(A))$. $V$ is a subvector space of $\mathbb{R}^{r}$ of dimension $\operatorname{dim} f(A)$. We consider the $\ell^{\infty}$-norm $\|\cdot\|_{\infty}$ on $\mathbb{R}^{r}$.

Claim 6.3. There exists a continuous homomorphism $g: V \rightarrow A$ satisfying $f \circ g=\left.\pi\right|_{V}$.

Proof. Let $M$ be the Pontyagin dual of $f(A)$. The dual group $\hat{V}$ of $V$ (we denote Pontragin duality by hat) is identified with $M \otimes \mathbb{R}$ and $\left(\left.\pi\right|_{V}\right)^{\wedge}(m)=m \otimes 1$ for $m \in M$. It is enough to construct a homomorphism $h: \hat{A} \rightarrow M \otimes \mathbb{R}$ satisfying $h \circ \hat{f}(m)=m \otimes 1$ for $m \in M$. (Note that every homomorphism defined on $\hat{A}$ automatically becomes continuous because its topology is discrete.)

Since the map $\hat{f}: M \rightarrow \hat{A}$ is injective,

$$
\hat{f} \otimes \mathrm{id}: M \otimes \mathbb{R} \rightarrow \hat{A} \otimes \mathbb{R}
$$


is also injective. Take an $\mathbb{R}$-linear map $\varphi: \hat{A} \otimes \mathbb{R} \rightarrow M \otimes \mathbb{R}$ satisfying $\varphi \circ(\hat{f} \otimes \mathrm{id})=\operatorname{id}_{M \otimes \mathbb{R}}$. Then the map $h: \hat{A} \rightarrow M \otimes \mathbb{R}$ defined by $h(a)=\varphi(a \otimes 1)$ satisfies $h \circ \hat{f}(m)=m \otimes 1$ for $m \in M$.

Let $B_{1 / 4}(V)$ be the closed 1/4-ball of $V$ around the origin. Note that the map $\pi:\left(B_{1 / 4}\left(\mathbb{R}^{r}\right),\|\cdot\|_{\infty}\right) \rightarrow\left(\mathbb{T}^{r}, \rho_{r}\right)$ is an isometry. So for $x, y \in B_{1 / 4}(V)$

$$
\|x-y\|_{\infty}=\rho_{r}(f(g(x)), f(g(y)) \leq \mathbf{d}(g(x), g(y)) .
$$

This implies

$$
\operatorname{Widim}_{\varepsilon}\left(B_{1 / 4}(V),\|\cdot\|_{\infty}\right) \leq \operatorname{Widim}_{\varepsilon}(A, \mathbf{d}) .
$$

We have $\operatorname{Widim}_{\varepsilon}\left(B_{1 / 4}(V),\|\cdot\|_{\infty}\right)=\operatorname{dim} V=\operatorname{dim} f(A)$ for $0<\varepsilon<1 / 4$ by (2.2) in Example 2.1 in \$2.1. This shows (6.2). We can prove (6.3) in the same way by using (2.3) in Example 2.1.

Proof of (6.1). Let $\mathcal{X} \subset\left(\mathbb{T}^{r}\right)^{\mathbb{Z}}$ be an algebraic action. First we prove $\operatorname{mdim}(\mathcal{X}, \sigma) \geq \operatorname{prodim}(\mathcal{X})$. Consider the projection $\pi_{N}: \mathcal{X} \rightarrow\left(\mathbb{T}^{r}\right)^{N}=$ $\mathbb{T}^{r N}$. This satisfies $\rho_{r N}\left(\pi_{N}(x), \pi_{N}(y)\right) \leq d_{N}(x, y)$. So we can use Lemma 6.2 and get

$$
\operatorname{Widim}_{\varepsilon}\left(\mathcal{X}, d_{N}\right) \geq \operatorname{dim} \pi_{N}(\mathcal{X}), \quad(0<\varepsilon<1 / 4) .
$$

Divide this by $N$ and take the limits with respect to $N$ and then $\varepsilon$. We get $\operatorname{mdim}(\mathcal{X}, \sigma) \geq \operatorname{prodim}(\mathcal{X})$.

Next we prove $\overline{\operatorname{mdim}}_{\mathrm{M}}(\mathcal{X}, \sigma, d) \leq \operatorname{prodim}(\mathcal{X})$. This completes the proof of (6.1) because metric mean dimension always dominates mean dimension (Theorem 2.2). Notice that the following map is an isometric embedding

$$
\begin{aligned}
\left(\pi_{M+N}(\mathcal{X}), \rho_{r(M+M)}\right) & \rightarrow\left(\pi_{M}(\mathcal{X}) \times \pi_{N}(\mathcal{X}), \rho_{r M} \times \rho_{r N}\right), \\
\pi_{M+N}(x) & \mapsto\left(\pi_{M}(x), \pi_{N}\left(\sigma^{M} x\right)\right)
\end{aligned}
$$

where the metric of the right-hand side is given by

$$
\rho_{r M} \times \rho_{r N}((x, y),(z, w))=\max \left(\rho_{r M}(x, z), \rho_{r N}(y, w)\right) .
$$

It follows that $\#\left(\pi_{N}(\mathcal{X}), \rho_{r N}, \varepsilon\right)$ is subadditive in $N$ and hence for any $\varepsilon>0$

$$
\lim _{N \rightarrow \infty} \frac{\log \#\left(\pi_{N}(\mathcal{X}), \rho_{r N}, \varepsilon\right)}{N}=\inf _{N>0} \frac{\log \#\left(\pi_{N}(\mathcal{X}), \rho_{r N}, \varepsilon\right)}{N}
$$

For $A \subset \mathbb{R}$, let $\pi_{A}: \mathcal{X} \rightarrow\left(\mathbb{T}^{r}\right)^{A \cap \mathbb{Z}}$ be the projection to $A \cap \mathbb{Z}$ coordinates. Let $\varepsilon>0$ and take $L=L(\varepsilon)>0$ satisfying $\sum_{|n|>L} 2^{-|n|}<$ 
$\varepsilon / 4$. Then

$$
\begin{aligned}
\#\left(\mathcal{X}, d_{N}, \varepsilon\right) & \leq \#\left(\pi_{[-L, N+L]}(\mathcal{X}), \rho_{r(N+2 L+1)}, \varepsilon / 4\right) \\
& =\#\left(\pi_{N+2 L+1}(\mathcal{X}), \rho_{r(N+2 L+1)}, \varepsilon / 4\right)
\end{aligned}
$$

Noting the above (6.4), we get

$$
\begin{aligned}
S(\mathcal{X}, \sigma, d, \varepsilon) & =\lim _{N \rightarrow \infty} \frac{\log \#\left(\mathcal{X}, d_{N}, \varepsilon\right)}{N} \\
& \leq \lim _{N \rightarrow \infty} \frac{\log \#\left(\pi_{N+2 L+1}(\mathcal{X}), \rho_{r(N+2 L+1)}, \varepsilon / 4\right)}{N} \\
& =\inf _{N>0} \frac{\log \#\left(\pi_{N}(\mathcal{X}), \rho_{r N}, \varepsilon / 4\right)}{N}
\end{aligned}
$$

Thus for any $N>0$

$$
\begin{aligned}
\overline{\operatorname{mdim}}_{\mathrm{M}}(\mathcal{X}, \sigma, d) & =\limsup _{\varepsilon \rightarrow 0} \frac{S(\mathcal{X}, \sigma, d, \varepsilon)}{\log (1 / \varepsilon)} \\
& \leq \frac{1}{N} \limsup _{\varepsilon \rightarrow 0} \frac{\log \#\left(\pi_{N}(\mathcal{X}), \rho_{r N}, \varepsilon / 4\right)}{\log (1 / \varepsilon)}
\end{aligned}
$$

$\pi_{N}(\mathcal{X})$ is a closed subgroup of $\mathbb{T}^{r N}$ and hence a smooth submanifold. Then the upper Minkowski dimension

$$
\overline{\operatorname{dim}}_{\mathrm{M}}\left(\pi_{N}(\mathcal{X}), \rho_{r N}\right)=\limsup _{\varepsilon \rightarrow 0} \frac{\log \#\left(\pi_{N}(\mathcal{X}), \rho_{r N}, \varepsilon\right)}{\log (1 / \varepsilon)}
$$

is equal to the topological dimension $\operatorname{dim} \pi_{N}(\mathcal{X})$. Thus for any $N$

$$
\overline{\operatorname{mdim}}_{\mathrm{M}}(\mathcal{X}, \sigma, d) \leq \frac{\operatorname{dim} \pi_{N}(\mathcal{X})}{N}
$$

Let $N \rightarrow \infty$. This proves $\overline{\operatorname{mdim}}_{\mathrm{M}}(\mathcal{X}, \sigma, d) \leq \operatorname{prodim}(\mathcal{X})$.

For any $N>0$ we define a distance $\bar{d}_{N}$ on $\left(\mathbb{T}^{r}\right)^{\mathbb{Z}}$ by (see $\S 4.2$ )

$$
\bar{d}_{N}(x, y)=\frac{1}{N} \sum_{n=0}^{N-1} d\left(\sigma^{n} x, \sigma^{n} y\right)
$$

Lemma 6.4. Let $\mathcal{X} \subset\left(\mathbb{T}^{r}\right)^{\mathbb{Z}}$ be an algebraic action. For any $0<\delta<1$ there exists $\varepsilon_{0}=\varepsilon_{0}(\delta)>0$ such that for any $0<\varepsilon<\varepsilon_{0}$ and $N>0$

$$
\#_{\text {sep }}\left(\mathcal{X}, \bar{d}_{N}, \varepsilon\right) \geq 4^{-N}(1 / \varepsilon)^{(1-\delta) \operatorname{dim} \pi_{N}(\mathcal{X})} .
$$

Recall that $\#_{\text {sep }}\left(\mathcal{X}, \bar{d}_{N}, \varepsilon\right)$ is the maximum cardinality of $\left\{x_{1}, \ldots, x_{n}\right\} \subset$ $\mathcal{X}$ satisfying $\bar{d}_{N}\left(x_{i}, x_{j}\right) \geq \varepsilon$ for $i \neq j$. 
Proof. We use (2.1) in $\$ 2.1$ and the estimate in Remark 4.8: For any $L>0$

$$
\begin{aligned}
\log \#_{\text {sep }}\left(\mathcal{X}, \bar{d}_{N}, \varepsilon\right) & \geq \log \#\left(\mathcal{X}, \bar{d}_{N}, 3 \varepsilon\right) \\
& \geq \log \#\left(\mathcal{X}, d_{N}, 6 L \varepsilon\right)-N-\frac{N}{L} \log \#(\mathcal{X}, d, 3 \varepsilon) .
\end{aligned}
$$

Let $L=(1 / 24)(1 / \varepsilon)^{\delta}$. Then

$\log \#_{\mathrm{sep}}\left(\mathcal{X}, \bar{d}_{N}, \varepsilon\right) \geq \log \#\left(\mathcal{X}, d_{N}, \frac{\varepsilon^{1-\delta}}{4}\right)-N-24 N \varepsilon^{\delta} \log \#(\mathcal{X}, d, 3 \varepsilon)$.

$(\mathcal{X}, d)$ has the tame growth of covering numbers (see Example 3.9). So there exists $\varepsilon_{0}>0$ so that for any $0<\varepsilon<\varepsilon_{0}$

$$
\frac{\varepsilon^{1-\delta}}{4}<\frac{1}{4}, \quad \varepsilon^{\delta} \log \#(\mathcal{X}, d, 3 \varepsilon)<\frac{1}{24} .
$$

By applying Lemma 6.2 to $\pi_{N}: \mathcal{X} \rightarrow\left(\mathbb{T}^{r}\right)^{N}$,

$$
\#\left(\mathcal{X}, d_{N}, \frac{\varepsilon^{1-\delta}}{4}\right) \geq\left(\frac{1}{\varepsilon^{1-\delta}}\right)^{\operatorname{dim} \pi_{N}(\mathcal{X})} \quad\left(0<\varepsilon<\varepsilon_{0}\right) .
$$

Combining these estimates we get

$$
\log \#_{\text {sep }}\left(\mathcal{X}, \bar{d}_{N}, \varepsilon\right) \geq(1-\delta) \operatorname{dim} \pi_{N}(\mathcal{X}) \log (1 / \varepsilon)-2 N .
$$

This is equivalent to the statement. (Recall that the base of the logarithm is two.)

Proof of Proposition 6.1. We know from Proposition 3.2 and (6.1) that

$$
\overline{\operatorname{rdim}}(\mathcal{X}, \sigma, d, \mu) \leq \operatorname{mdim}_{\mathrm{M}}(\mathcal{X}, \sigma, d)=\operatorname{prodim}(\mathcal{X}) .
$$

So it is enough to prove $\underline{\operatorname{rim}}(\mathcal{X}, \sigma, d, \mu) \geq \operatorname{prodim}(\mathcal{X})$. We can assume $\operatorname{prodim}(\mathcal{X})>0$

Recall that the distance $d$ is homogeneous. In particular the measure $\mu\left(B_{r}^{\circ}\left(x, \bar{d}_{N}\right)\right)$ of the open ball around $x \in \mathcal{X}$ is independent of $x$. So we denote it by $\mu\left(B_{r}^{\circ}\left(\bar{d}_{N}\right)\right)$.

Let $\left\{x_{1}, \ldots, x_{K}\right\}$ be a separated set of $\left(\mathcal{X}, \bar{d}_{N}\right)$ with $K=\#_{\text {sep }}\left(\mathcal{X}, \bar{d}_{N}, \varepsilon\right)$. Since the balls $B_{\varepsilon / 2}^{\circ}\left(x_{i}, \bar{d}_{N}\right)$ are disjoint with each other, $K \mu\left(B_{\varepsilon / 2}^{\circ}\left(\bar{d}_{N}\right)\right) \leq$ 1. Let $0<\delta<1 / 2$. It follows from Lemma 6.4 that for $0<\varepsilon<\varepsilon_{0}(\delta)$

$$
\mu\left(B_{\varepsilon / 2}^{\circ}\left(\bar{d}_{N}\right)\right) \leq K^{-1} \leq 4^{N} \varepsilon^{(1-\delta) \operatorname{dim} \pi_{N}(\mathcal{X})} .
$$

Since $\operatorname{dim} \pi_{N}(\mathcal{X})$ is subadditive in $N$,

$$
\operatorname{prodim}(\mathcal{X})=\lim _{N \rightarrow \infty} \frac{\operatorname{dim} \pi_{N}(\mathcal{X})}{N}=\inf _{N>0} \frac{\operatorname{dim} \pi_{N}(\mathcal{X})}{N}
$$


and we assumed that this is positive. Therefore there exists $\varepsilon_{1}=$ $\varepsilon_{1}(\delta)>0$ such that for any $0<\varepsilon<\varepsilon_{1}$

$$
\mu\left(B_{\varepsilon / 2}^{\circ}\left(\bar{d}_{N}\right)\right) \leq(\varepsilon / 2)^{N(1-2 \delta) \operatorname{prodim}(\mathcal{X})} .
$$

This implies that for all $E \subset \mathcal{X}$ with $\operatorname{diam}\left(E, \bar{d}_{N}\right)<\varepsilon_{1} / 2$

$$
\mu(E) \leq\left(\operatorname{diam}\left(E, \bar{d}_{N}\right)\right)^{N(1-2 \delta) \operatorname{prodim}(\mathcal{X})} .
$$

We use Lemma 4.5 in $\$ 4.2$ and get

$$
\underline{\operatorname{rdim}}(\mathcal{X}, \sigma, d, \mu) \geq(1-2 \delta) \operatorname{prodim}(\mathcal{X}) .
$$

Let $\delta \rightarrow 0$. This proves $\underline{\operatorname{rdim}}(\mathcal{X}, \sigma, d, \mu) \geq \operatorname{prodim}(\mathcal{X})$.

\section{REFERENCES}

[B71] T. Berger, Rate distortion theory: A mathematical basis for data compression, Englewood Cliffs, NJ: Prentice-Hall, 1971.

[CT06] T. M. Cover, J. A. Thomas, Elements of information theory, second edition, Wiley, New York, 2006.

[D70] E. I. Dinaburg, A correlation between topological entropy and metric entropy, Dokl. Akad. Nauk SSSR 190 (1970) 19-22.

[ECG94] M. Effros, P. A. Chou, G. M. Gray, Variable-rate source coding theorems for stationary nonergodic sources, IEEE Trans. Inf. Theory vol. 40, pp. 1920$1925,1994$.

[EW11] M. Einsiedler, T. Ward, Ergodic theory with a view towards number theory, Graduate Texts in Mathematics 259, Springer, London.

[F67] H. Furstenberg, Disjointness in ergodic theory, minimal sets, and a problem in Diophantine approximation, Math. Systems Theory 1 (1967) 1-49.

[Goodm71] T. N. T. Goodman, Relating topological entropy and measure entropy, Bull. London Math. Soc. 3 (1971) 176-180.

[Goodw69] L. W. Goodwyn, Topological entropy bounds measure-theoretic entropy, Proc. Amer. Math. Soc. 23 (1969) 679-688.

[Gra90] R.M. Gray, Entropy and information theory, New York, Springer-Verlag, 1990.

[Gro99] M. Gromov, Topological invariants of dynamical systems and spaces of holomorphic maps: I, Math. Phys. Anal. Geom. vol. 2 pp. 323-415, 1999.

[Gut12] Y. Gutman, Mean dimension and Jaworski-type theorems, Proceedings of the London Mathematical Society 111(4) (2015) 831-850.

[GLT16] Y. Gutman, E. Lindenstrauss, M. Tsukamoto, Mean dimension of $\mathbb{Z}^{k}$ actions, Geom. Funct. Anal. 26 Issue 3 (2016) 778-817.

[GQT] Y. Gutman, Y. Qiao, M. Tsukamoto, Application of signal analysis to the embedding problem of $\mathbb{Z}^{k}$-actions, preprint, arXiv:1709.00125.

[GT] Y. Gutman , M. Tsukamoto, Embedding minimal dynamical systems into Hilbert cubes, preprint, arXiv:1511.01802,

[H95] J. D. Howroyd, On dimension and on the existence of sets of finite, positive Hausdorff measures, Proc. London Math. Soc. 70 (1995) 581-604.

[J70] R. I. Jewett, The prevalence of uniquely ergodic systems, J. Math. Mech. 19 (1970) 717-729. 
[KD94] T. Kawabata and A. Dembo, The rate distortion dimension of sets and measures, IEEE Trans. Inf. Theory, vol. 40, no. 5, pp. 1564-1572, Sep. 1994.

[KT63] A. N. Kolmogorov and V. M. Tihomirov, $\varepsilon$-entropy and $\varepsilon$-capacity of sets in functional spaces, Amer. Math. Soc. Transl. vol. 33, ser. 2, pp. 277-367, 1963.

[Kri70] W. Krieger, On unique ergodicity, Proc. sixth Berkeley symposium, Math. Statist. Probab. Univ. of California Press, 1970, 327-346.

[LDN79] A. Leon-Garcia, L. D. Davisson, D. L. Neuhoff, New results on coding of stationary nonergodic sources, IEEE Trans. Inform. Theory, vol. 25, pp. 137-144, 1979.

[LL18] H. Li, B. Liang, Mean dimension, mean rank and von Neumann-Lück rank, J. Reine Angew. Math. 739 (2018) 207-240.

[Lin99] E. Lindenstrauss, Mean dimension, small entropy factors and an embedding theorem, Inst. Hautes Études Sci. Publ. Math. vol. 89 pp. 227-262, 1999.

[LT14] E. Lindenstrauss, M. Tsukamoto, Mean dimension and an embedding problem: an example, Israel J. Math. 199 (2014) 573-584.

[LT18] E. Lindenstrauss, M. Tsukamoto, From rate distortion theory to metric mean dimension: variational principle, IEEE Trans. Inf. Theory, vol. 64, No. 5, pp. 3590-3609, May, 2018.

[LW00] E. Lindenstrauss, B. Weiss, Mean topological dimension, Israel J. Math. vol. 115 pp. 1-24, 2000.

[Mat95] P. Mattila, Geometry of sets and measures in Euclidean spaces, Fractals and rectifiability, Cambridge Studies in Advanced Mathematics, 44, Cambridge University Press, Cambridge, 1995.

[MT] T. Meyerovitch, M. Tsukamoto, Expansive multiparameter actions and mean dimension, arXiv:1710.09647, to appear in Trans. Amer. Math. Soc.

[Mis76] M. Misiurewicz, A short proof of the variational principle for $\mathbb{Z}_{+}^{N}$ actions on a compact space, International Conference on Dynamical Systems in Mathematical Physics (Rennes, 1975), Astérisque, vol. 40, pp. 145-157, Soc. Math. France, Paris, 1976.

[PS32] L. Pontrjagin, L. Schnirelmann, Sur une propriété métrique de la dimension, Ann. Math. 33 (1932) 152-162.

[Rén59] A. Rényi, On the dimension and entropy of probability distributions, Acta Math. Sci. Hung. vol. 10, pp. 193-215, 1959.

[RJEP] F. E. Rezagah, S. Jalali, E. Erkip and H. V. Poor, Rate-distortion dimension of stochastic processes, arXiv:1607.06792

[Sch95] K. Schmidt, Dynamical systems of algebraic origin, Progress in Mathematics, 128, Birkhäuser Verlag, Basel, 1995.

[Sh48] C. E. Shannon, A mathematical theory of communication, Bell Syst. Tech. J. 27 (1948) 379-423, 623-656.

[Sh59] C. E. Shannon, Coding theorems for a discrete source with a fidelity criterion, IRE Nat. Conv. Rec., Pt. 4, pp. 142-163, 1959.

[T09] M. Tsukamoto, Deformation of Brody curves and mean dimension, Ergod. theory Dyn. Syst. 29 (2009) 1641-1657.

[T18a] M. Tsukamoto, Mean dimension of the dynamical system of Brody curves, Invent. math. 211 (2018) 935-968.

[T18b] M. Tsukamoto, Large dynamics of Yang-Mills theory: mean dimension formula, J. Anal. Math. 134 (2018) 455-499. 
[VV] A. Velozo, R. Velozo, Rate distortion theory, metric mean dimension and measure theoretic entropy, arXiv:1707.05762,

[Vil09] C. Villani, Optimal transport old and new, Springer-Verlag, Berlin, 2009.

[WV10] Y. Wu and S. Verdú, Rényi information dimension: fundamental limits of almost lossless analogue compression, IEEE Trans. Inf. Theory, vol. 56, no. 8, (2010) 3721-3747.

\section{Elon Lindenstrauss}

Einstein Institute of Mathematics, Hebrew University, Jerusalem 91904, Israel

E-mail address: elon@math.huji.ac.il

Masaki Tsukamoto

Department of Mathematics, Kyoto University, Kyoto 606-8502, Japan

E-mail address: masaki.tsukamoto@gmail.com 